\title{
Alternarin A, a Drimane Meroterpenoid Suppresses Neuronal Excitabil- ity from the Coral-Associated Fungi Alternaria sp. ZH-15
}

\author{
Hong-Liang Wang,, ,,\#\# Ran Li, ${ }^{+, \#}$ Jiao Li, ${ }^{\perp, \#}$ Jing He, ' Zheng-Yu Cao, ' Tibor Kurtán, ${ }^{\nabla}$ Attila Mándi,, \\ Gui-Liang Zheng, ${ }^{*}, 0$ and Wen Zhang ${ }^{*},+, \$$
}

†School of Pharmacy, Second Military Medical University, 325 Guo-He Rd., Shanghai 200433, People’s Republic of China ‡NCO School of Army Medical University, 450 West Zhong-Shan Rd., Shijiazhuang 050000, People’s Republic of China

${ }^{\perp}$ College of Pharmaceutical Science, Zhejiang Chinese Medical University, Gao-Ke Rd., Hangzhou 311402, People's Republic of China

I State Key Laboratory of Natural Medicines and Department of TCM Pharmacology, School of Traditional Chinese Pharmacy, China Pharmaceutical University, 639 Long-Mian Ave., Nanjing 211198, People’s Republic of China

${ }^{\nabla}$ Department of Organic Chemistry, University of Debrecen, POB 400, H-4002 Debrecen, Hungary

oDepartment of Otorhinolaryngology, Head and Neck Surgery, Xinhua Hospital Affiliated to Shanghai Jiao Tong University School of Medicine, 1665 Kong-Jiang Rd., Shanghai 200092, People’s Republic of China

§Tongji Hospital Affiliated to Tongji University, 389 Xin-Cun Rd., Shanghai 200065, People’s Republic of China 


\section{Table of contents}

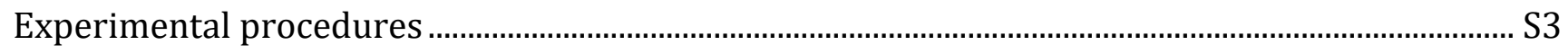

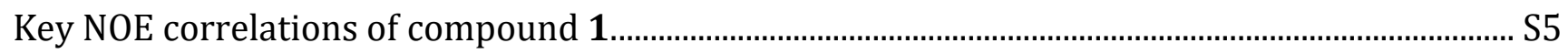

Low-energy conformers of truncated model compound 4 ……………………….............................. S6

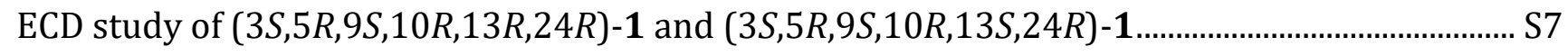

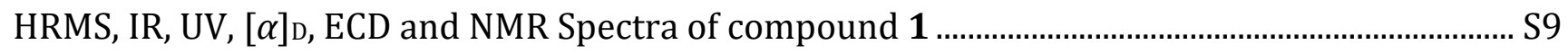

Computational data for low-energy conformers of truncated model compound $\mathbf{4}$ and the two

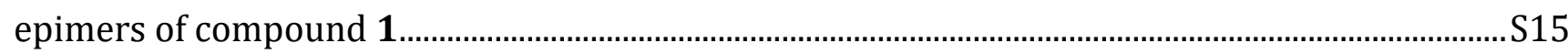

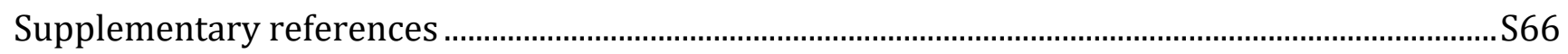




\section{Experimental Procedures.}

\subsection{General Experimental Procedures.}

Optical rotations were recorded by an Autopol VI polarimeter and ECD spectra was recorded by JASCO-715 spectropolarimeter. UV spectra were measured by Varian Cary 300 Bio UV-Visible. The NMR spectra was acquired by Bruker DRX 600 spectrometer. For chemical shifts, residual $\mathrm{CDCl}_{3}$ signal $\left(\delta_{\mathrm{H}} 7.26 \mathrm{ppm} ; \delta_{\mathrm{c}} 77.0 \mathrm{ppm}\right)$ was used as reference. HRMS was obtained by an Agilent Q-TOF Micro mass spectrometer. Column chromatography (CC) was conducted on commercial silica gel (200-300 mesh and 300-400 mesh, Huanghai, Yantai). Semipreparative HPLC was achieved through an Agilent 1100 system with an YMC Pack ODS-A column $(250 \times 10 \mathrm{~mm}, 5 \mu \mathrm{m})$. TLC was carried out on precoated silica gel plate (HSGF-254, Huanghai, Yantai), and spots on TLC could be detected under UV or by spraying with $10 \%$ anisaldehyde in $\mathrm{H}_{2} \mathrm{SO}_{4}$.

\subsection{Fungal Material, Fermentation and Isolation.}

The fungus was isolated from the internal tissues of the soft coral Lobophytum crassum collected from the Dongsha Atoll in the South China Sea at a depth of 15 m, in Sep. 2016, and was identified as Alternaria sp. ZH-15 (GenBank accession number 2081031) by sequence analysis of the ITS region of the rDNA. A voucher sample (internal strain No. ZH-15 II ) has been deposited in the Second Military Medical University, Shanghai, People's Republic of China.

The fungus Alternaria sp. ZH-15 was cultivated on $25 \mathrm{~L}$ of 5\% w/v biomalt (Villa Natura, Germany) solid agar medium (20 g/L biomalt, $15 \mathrm{~g} / \mathrm{L}$ agar, $800 \mathrm{~mL} / \mathrm{L} \mathrm{ASW})$ at room temperature for 28 days. The culture medium was extracted with ethyl acetate to afford a residue (27.0 g) after removal of the solvent under reduced pressure. The crude extract as subjected to a column chromatography (CC) on silica gel eluted with a gradient of methanol in dichloromethane $\left(\mathrm{MeOH} / \mathrm{CH}_{2} \mathrm{Cl}_{2}\right.$ 1:100 to 1:1) to give 11 fractions (Fr.s 1-11). Fr.6 was purified by CC on Sephadex LH-20 $\left(\mathrm{CH}_{2} \mathrm{Cl}_{2} / \mathrm{MeOH}\right.$ 2:1) to give four subfractions (Fr.6.1-6.4). Fr.6.2 was further separated by $\mathrm{CC}$ on silica gel $\left(\mathrm{CH}_{2} \mathrm{Cl}_{2} / \mathrm{MeOH} 40: 1\right)$ followed by HPLC to afford compounds $2\left(1.4 \mathrm{mg}, \mathrm{MeOH}_{\mathrm{H}} \mathrm{O}, 72: 28,1.5 \mathrm{~mL} / \mathrm{min}, t_{\mathrm{R}}=36.5 \mathrm{~min}\right)$ and $3\left(1.5 \mathrm{mg}, \mathrm{MeOH} / \mathrm{H}_{2} \mathrm{O}\right.$, 68:32, $\left.1.5 \mathrm{~mL} / \mathrm{min}, t_{\mathrm{R}}=25.0 \mathrm{~min}\right)$. Fr.8 was separated by CC on Sephadex LH-20 $\left(\mathrm{CH}_{2} \mathrm{Cl}_{2} / \mathrm{MeOH}\right.$ 2:1) to give five subfractions (Fr.8.1-8.5). Fr.8.4 was purified by CC on silica gel $\left(\mathrm{CH}_{2} \mathrm{Cl}_{2} / \mathrm{MeOH} 50: 1\right)$ followed by HPLC to afford $\mathbf{1}$ (12.3 mg, $\left.\mathrm{MeOH} / \mathrm{H}_{2} \mathrm{O}, 75: 25,1.5 \mathrm{~mL} / \mathrm{min}, t_{\mathrm{R}}=19.0 \mathrm{~min}\right)$.

Alternarin A (1): pale gum (MeOH); $\mathrm{Rf}_{\mathrm{f}} 0.4\left(\mathrm{CH}_{2} \mathrm{Cl}_{2} / \mathrm{MeOH}, 15: 1\right) ;[\alpha]_{\mathrm{D}}^{25}-117.8\left(c\right.$ 0.27, $\left.\mathrm{CHCl}_{3}\right) ; \mathrm{UV}(\mathrm{MeCN}) \lambda_{\max }(\log \varepsilon)$ $192 \mathrm{~nm}$ (1.47), 214 (0.60), 249(0.17); ECD (MeCN, c $\left.5.5 \times 10^{-4}\right) \lambda_{\max }(\Delta \varepsilon) 290(-0.9), 255(+2.0), 210$ ( -1.7) nm; IR (film) $\nu_{\max } 3377,2956,2932,2871,1723,1712,1643,1387,1178,1009 \mathrm{~cm}^{-1} .{ }^{1} \mathrm{H}$ and ${ }^{13} \mathrm{C}$ NMR data, see Table 1 ; HRMS (ESI) $m / z[\mathrm{M}+\mathrm{Na}]^{+}$Calcd for $\mathrm{C}_{26} \mathrm{H}_{38} \mathrm{O}_{7} \mathrm{SNa}$ 517.2236; Found 517.2237.

Macrophorin A (2): white amorphous powder $\left(\mathrm{CH}_{2} \mathrm{Cl}_{2}\right) ;[\alpha]_{\mathrm{D}}^{25}+20.6(c 0.14, \mathrm{MeOH}) ;{ }^{1} \mathrm{H} \mathrm{NMR}\left(600 \mathrm{MHz}, \mathrm{CDCl}_{3}\right) \delta_{\mathrm{H}} 1.18$ $(1 \mathrm{H}, \mathrm{ddd}, J=13.5,13.5,4.3 \mathrm{~Hz}, \mathrm{H}-1 \alpha), 1.78(1 \mathrm{H}, \mathrm{ov}, \mathrm{H}-1 \beta), 1.53(1 \mathrm{H}, \mathrm{m}, \mathrm{H}-2 \alpha), 1.58(1 \mathrm{H}, \mathrm{m}, \mathrm{H}-2 \beta), 1.41(1 \mathrm{H}, \mathrm{ddd}, J=$

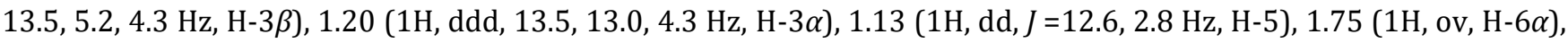
1.31 (1H, dddd, $J=12.6,12.6,4.3,4.3 \mathrm{~Hz}, \mathrm{H}-6 \beta), 1.98$ (1H, ov, H-7 $\alpha$ ), 2.37 (1H, ov, H-7 $\beta$ ), $1.76(1 \mathrm{H}, \mathrm{ov}, \mathrm{H}-9), 2.37$ (1H, ov, H-11a), 1.99 (1H, ov, H-11b), 4.82 (1H, s, H-12a), 4.55(1H, s, H-12b), 0.87 (3H, s, H-13), 0.80 (3H, s, H-14), 0.71 (3H, s, H-15), 5.99 (1H, s, H-2'), 3.73 (1H, d, J = 1.3 Hz, H-5'), 4.60 (1H, br s, H-4'), 4.50 (1H, d, J = 15.5 Hz, H-7'a), 4.31 (1H, d, J 
$=15.5 \mathrm{~Hz}, \mathrm{H}-7 \mathrm{\prime})$ ); ${ }^{13} \mathrm{C}$ NMR $\left(150 \mathrm{MHz}, \mathrm{CDCl}_{3}\right) \delta_{\mathrm{C}} 39.1\left(\mathrm{CH}_{2}, \mathrm{C}-1\right), 19.4\left(\mathrm{CH}_{2}, \mathrm{C}-2\right), 42.1\left(\mathrm{CH}_{2}, \mathrm{C}-3\right), 33.8(\mathrm{C}, \mathrm{C}-4), 55.7(\mathrm{CH}$, C-5), 24.5 ( $\left.\mathrm{CH}_{2}, \mathrm{C}-6\right), 38.3$ (CH $2, \mathrm{C}-7$ ), 149.8 (C, C-8), 51.6 (CH, C-9), 39.8 (C, C-10), $21.0\left(\mathrm{CH}_{2}, \mathrm{C}-11\right), 106.7$ (CH2, C-12), $33.6\left(\mathrm{CH}_{3}, \mathrm{C}-13\right), 21.7\left(\mathrm{CH}_{3}, \mathrm{C}-14\right), 14.5\left(\mathrm{CH}_{3}, \mathrm{C}-15\right), 193.8$ (C, C-1'), 122.5 (CH, C-2'), 155.2 (C, C-3'), 64.6 (CH, C-4'), 62.3 (CH, C-5'), 59.5 (C, C-6'), $64.2\left(\mathrm{CH}_{2}, \mathrm{C}^{-} \mathbf{7}^{\prime}\right)$. MS (ESI) $\mathrm{m} / \mathrm{z}[\mathrm{M}+\mathrm{Na}]^{+} 383.2$.

Macrophorin B (3): white amorphous powder $\left(\mathrm{CH}_{2} \mathrm{Cl}_{2}\right) ;[\alpha]_{\mathrm{D}}^{25}+55.1(c 0.08, \mathrm{MeOH}) ;{ }^{1} \mathrm{H} \mathrm{NMR}\left(600 \mathrm{MHz}, \mathrm{CDCl}_{3}\right) \delta_{\mathrm{H}} 1.68$ $(1 \mathrm{H}$, ov H-1 $\alpha), 2.06(1 \mathrm{H}, \mathrm{ov}, \mathrm{H}-1 \beta), 2.39(1 \mathrm{H}, \mathrm{ov}, \mathrm{H}-2 \alpha), 2.62(1 \mathrm{H}, \mathrm{ddd}, J=15.2,12.8,6.3 \mathrm{~Hz}, \mathrm{H}-2 \beta), 1.62(1 \mathrm{H}, \mathrm{dd}, J=12.8$, $2.7 \mathrm{~Hz}, \mathrm{H}-5), 1.68$ (1H, ov, H-6 $\alpha$ ), 1.48 (1H, dddd, $J=12.8,12.8,12.8,4.1 \mathrm{~Hz}, \mathrm{H}-6 \beta), 2.00(1 \mathrm{H}, \mathrm{ddd}, J=12.8,12.8,4.4 \mathrm{~Hz}$, $\mathrm{H}-7 \alpha$ ), $2.40(1 \mathrm{H}, \mathrm{ov}, \mathrm{H}-7 \beta), 1.76(1 \mathrm{H}, \mathrm{d}, J=10.7 \mathrm{~Hz}, \mathrm{H}-9), 2.26$ (1H, d, $J=14.7 \mathrm{~Hz}, \mathrm{H}-11 \mathrm{a}), 2.10$ (1H, dd, J = 14.7, $10.7 \mathrm{~Hz}$, H-11b), 4.89 (1H, s, H-12a), 4.63 (1H, s, H-12b), 1.07 (3H, s, H-13), 1.00 (3H, s, H-14), 0.88 (3H, s, H-15), 5.97 (1H, s, H2'), 4.52 (1H, br s, H-4'), 3.70 (1H, s, H-5'), 4.48 (1H, d, J = 15.5 Hz, H-7'a), 4.25 (1H, d, J = 15.5 Hz, H-7'b); ${ }^{13}$ C NMR (150 $\left.\mathrm{MHz}, \mathrm{CDCl}_{3}\right) \delta_{\mathrm{C}} 37.6\left(\mathrm{CH}_{2}, \mathrm{C}-1\right), 34.8\left(\mathrm{CH}_{2}, \mathrm{C}-2\right), 217.4$ (C, C-3), 47.9 (C, C-4), $55.2(\mathrm{CH}, \mathrm{C}-5), 25.1\left(\mathrm{CH}_{2}, \mathrm{C}-6\right), 37.6\left(\mathrm{CH}_{2}, \mathrm{C}-\right.$ 7), 148.0 (C, C-8), 50.6 (CH, C-9), 39.3 (C, C-10), $21.2\left(\mathrm{CH}_{2}, \mathrm{C}-11\right), 108.2\left(\mathrm{CH}_{2}, \mathrm{C}-12\right), 26.1\left(\mathrm{CH}_{3}, \mathrm{C}-13\right), 21.8\left(\mathrm{CH}_{3}, \mathrm{C}-14\right)$, $14.2\left(\mathrm{CH}_{3}, \mathrm{C}-15\right), 194.4$ (C, C-1'), 121.9 (CH, C-2'), 156.5 (C, C-3'), 64.2 (CH, C-4'), 62.0 (CH, C-5'), 59.4(C, C-6'), 63.6(CH2, C-7'). MS (ESI) $m / z[\mathrm{M}+\mathrm{H}]^{+} 375.2$.

\subsection{Synthesis of $(R)$-MPA or (S)-MPA ethers of compound 1.}

Esterification of compound 1 with $(R)$-MPA. To the solution of $\mathbf{1}(0.50 \mathrm{mg})$ with $(R)$-MPA $(1.0 \mathrm{mg})$ and DMAP $(1.0 \mathrm{mg})$ in dry $\mathrm{CH}_{2} \mathrm{Cl}_{2}(1.0 \mathrm{~mL})$, was added EDCI $(1.0 \mathrm{mg})$. The suspension was stirred at room temperature overnight, and then purified by a mini silica gel column chromatography (300-400 mesh, petroleum ether/acetone, 10:1) afforded the $(R)$ MPA ester of 1 (0.4 mg, 51\%): selected ${ }^{1} \mathrm{H}$ NMR (600 MHz, $\left.\mathrm{CDCl}_{3}\right) \delta 3.63$ (d, $J=3.0 \mathrm{~Hz}, 2 \mathrm{H}, \mathrm{H}_{2}-18$ ), 3.62 (s, 3H, -OMe),

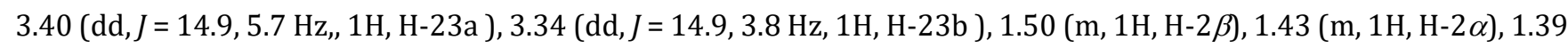

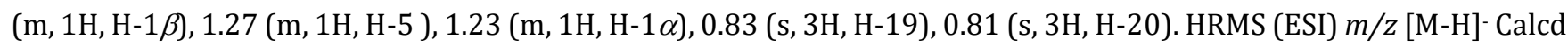
for $\mathrm{C}_{44} \mathrm{H}_{53} \mathrm{O}_{11} \mathrm{~S}$ 789.3309; Found 789.3316.

Esterification of compound 1 with $(S)$-MPA. Compound $\mathbf{1}(0.50 \mathrm{mg})$ was treated in the same way as mentioned above with $(S)$-MPA (1.0 mg), affording the (S)-MPA ester of $1(0.36 \mathrm{mg}, 46 \%)$ : selected ${ }^{1} \mathrm{H}$ NMR (600 MHz, $\left.\mathrm{CDCl}_{3}\right) \delta 3.74(\mathrm{~s}$, $3 \mathrm{H},-\mathrm{OMe}$ ), 3.49 (d, $J=3.0 \mathrm{~Hz}, 2 \mathrm{H}, \mathrm{H}-18$ ), 3.21 (dd, $J=14.9,3.8 \mathrm{~Hz}, 1 \mathrm{H}, \mathrm{H}-23 \mathrm{~b}$ ), 3.17 (dd, J = 14.9, $5.7 \mathrm{~Hz}, 1 \mathrm{H}, \mathrm{H}-23 \mathrm{a}$ ),

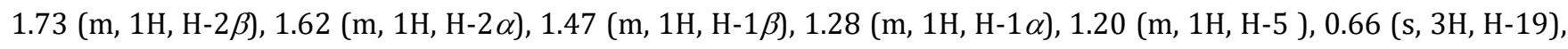
0.63 (s, 3H, H-20). HRMS (ESI) $m / z$ [M-H]- Calcd for $\mathrm{C}_{44} \mathrm{H}_{53} \mathrm{O}_{11} \mathrm{~S}$ 789.3309; Found 789.3322.

\subsection{Computational methods.}

Mixed torsional/low-mode conformational searches were carried out by means of the Macromodel 10.8.011 software using the MMFF with an implicit solvent model for $\mathrm{CHCl}_{3}$ applying a $21 \mathrm{~kJ} \mathrm{~mol}^{-1}$ energy window. ${ }^{1}$ Geometry reoptimizations of the resultant conformers [ $\omega$ B97X/TZVP with PCM solvent model for MeCN] and TDDFT ECD calculations were performed with Gaussian 09 using various functionals (B3LYP, BH\&HLYP, CAM-B3LYP, PBE0) and the TZVP basis set with the same solvent model as in the preceding DFT optimization step. ${ }^{2}$ ECD spectra were generated as the sum of Gaussians with $3000 \mathrm{~cm}^{-1}$ half-height widths, using dipole-velocity-computed rotational strengths. ${ }^{3}$ Boltzmann distributions were estimated from the $\omega$ B97X energies. The MOLEKEL program was used for visualization of the results. ${ }^{4}$ 


\subsection{Measurement of Synchronous Intracellular $\mathrm{Ca}^{2+}$ Oscillations.}

Primary neocortical neurons dissociated from the cortex from $\mathrm{C} 57 \mathrm{Bl} / 6 \mathrm{~J}$ mouse were cultured as described previously. ${ }^{5}$ Neocortical neurons at 9 days in vitro (DIV) were used to investigate the influence of meroterpenoids on intracellular $\mathrm{Ca}^{2+}$ concentration $\left(\left[\mathrm{Ca}^{2+}\right]_{\mathrm{i}}\right)$. Briefly, the neurons were loaded with Fluo- 4 for 1 hour at $37^{\circ} \mathrm{C}$ in Locke's buffer. After recording the baseline spontaneous $\mathrm{Ca}^{2+}$ oscillations for 5 minutes, meroterpenoids with different concentrations were added to the corresponding well, and the $\left[\mathrm{Ca}^{2+}\right]_{\mathrm{i}}$ was monitored for 15 minutes. To test anti-epileptic potential of the meroterpenoids, the epilepticus contributor, 4-AP $(10 \mu \mathrm{M})$, was then added and the monitoring for $\left[\mathrm{Ca}^{2+}\right]_{\mathrm{i}}$ was continued for additional 10 minutes. The presented data were values of $F / F_{0}$, where $F$ is the fluorescence intensity at any time point whereas $\mathrm{F}_{0}$ is the basal fluorescence. An event with $\Delta \mathrm{F} / \mathrm{F}_{0}$ over 0.1 units was considered to be an SCO. The frequency and amplitude of SCOs were quantified using Origin software (V7.0) from a time period of 5 min after compound addition.

\section{Key NOE correlations of compound 1.}

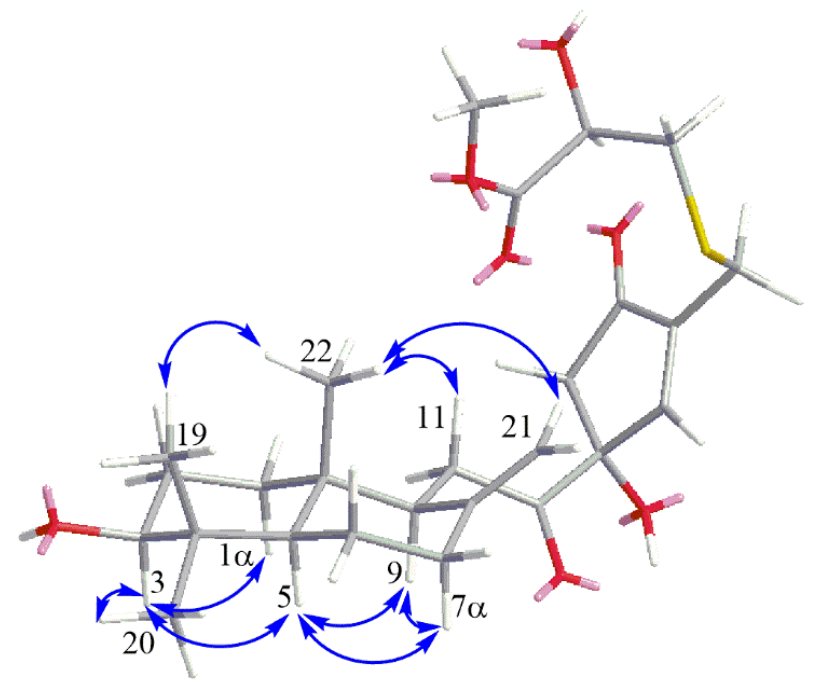

Figure S1. Key NOE correlations of compound 1. 


\section{Low-energy conformers of truncated model compound (R)-4-acetyl-4-hydroxy-2-(methyl- thiomethyl)cyclopent-2-enone (4).}
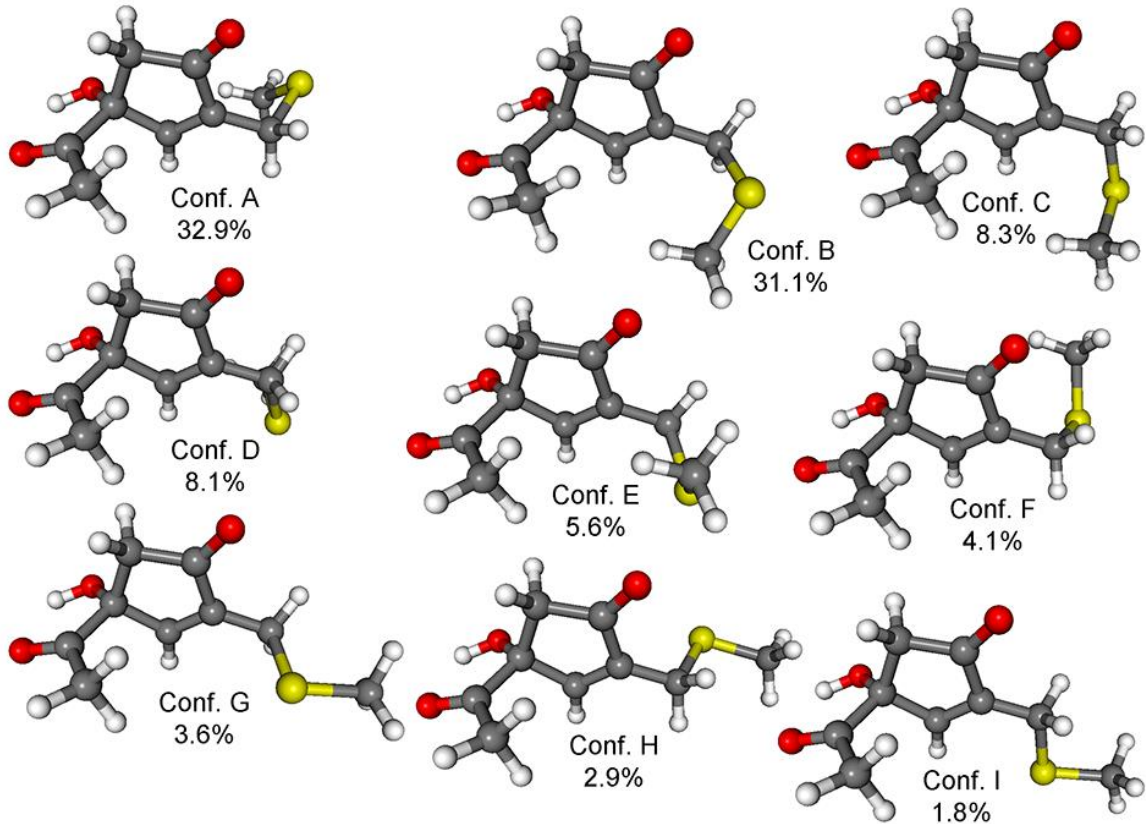

Figure S2. Structure and population of the low-energy $\omega$ B97X/TZVP PCM/MeCN conformers $(>1 \%)$ of $(R)$-4-acetyl-4hydroxy-2-(methylthiomethyl)cyclopent-2-enone (4), model compound for the ECD calculation of $\mathbf{1}$.

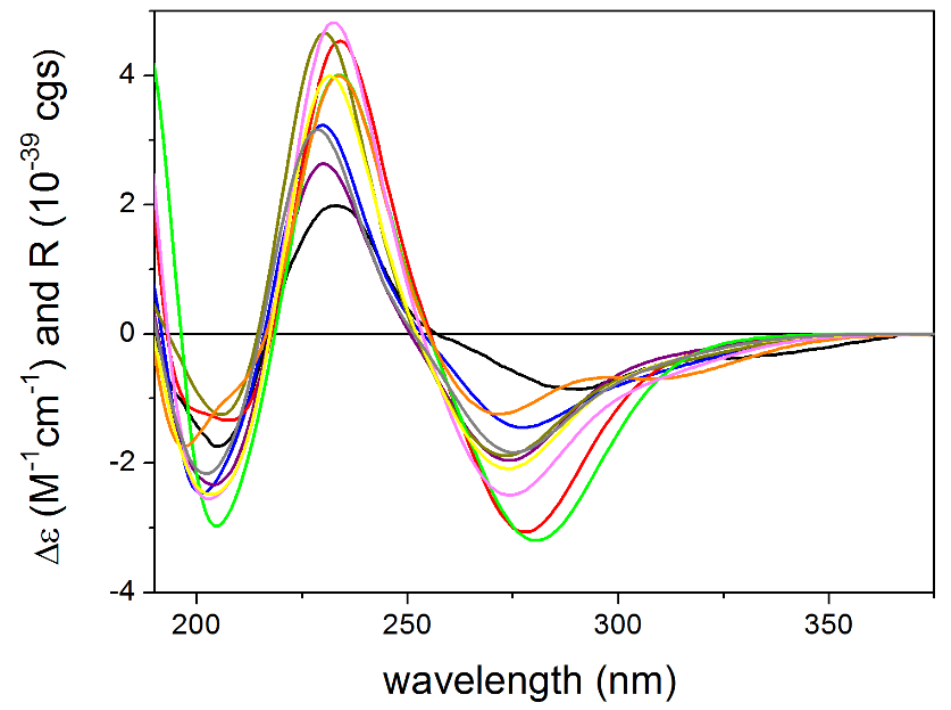

Figure S3. Experimental ECD spectrum of 1 in MeCN (black) compared with the BH\&HLYP/TZVP PCM/MeCN ECD spectra of the low-energy (> 1\%) $\omega$ B97X/TZVP PCM/MeCN conformers of $(R)$-4. Conf A: blue, Conf B: red, Conf C: purple, Conf D: dark yellow, Conf E: green, Conf F: orange, Conf G: pink, Conf H: yellow, Conf I: gray. 


\section{ECD study of $(3 S, 5 R, 9 S, 10 R, 13 R, 24 R)-1$ and $(3 S, 5 R, 9 S, 10 R, 13 S, 24 R)-1$.}

In order to confirm the ECD computational results performed on the model compound $(R)-\mathbf{4}$ and to justify truncations, the ECD calculation protocol was also performed on the epimeric $(3 S, 5 R, 9 S, 10 R, 13 R, 24 R)-1$ and $(3 S, 5 R, 9 S, 10 R, 13 S, 24 R)-1$. Since initial MMFF conformational search of $(3 S, 5 R, 9 S, 10 R, 13 R, 24 R)-1$ resulted in 2516 conformers, while that of the C-13 epimer produced 1568, the number of geometries for DFT re-optimization had to be reduced. ${ }^{6}$ Therefore the initial MMFF conformers were re-optimized first at the AM1 level and re-clustered by neglecting the orientation of the decalin moiety. The resulting 370 and 236 conformers were then re-optimized at the $\omega B 97 X / T Z V P$ PCM/MeCN level yielding 25 and 12 low-energy conformers above 1\% Boltzmann-population, respectively. While all the applied combinations of levels of $(3 S, 5 R, 9 S, 10 R, 13 R, 24 R)-1$ reproduced well the experimental spectrum of $\mathbf{1}$, the computed ECD spectra of $(3 S, 5 R, 9 S, 10 R, 13 S, 24 R)$-1 gave mirror-image agreement. Thus, the absolute configuration of 1 could be determined as $(3 S, 5 R, 9 S, 10 R, 13 R, 24 R)$, and ECD calculations proved that the absolute configuration of the $\mathrm{C}-13$ chirality center had decisive role on the ECD pattern.

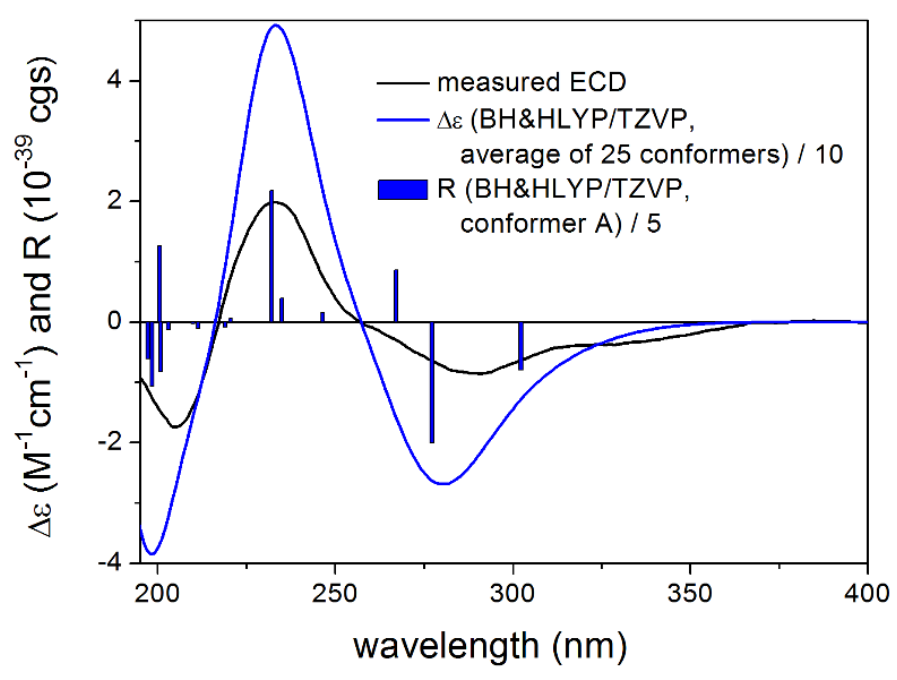

Figure S4. Experimental ECD spectrum of 1 in MeCN compared with the BH\&HLYP/TZVP PCM/MeCN ECD spectrum of $(3 S, 5 R, 9 S, 10 R, 13 R, 24 R)-1$ computed for the low-energy ( $\geq 1 \%) \omega B 97 X / T Z V P$ PCM/MeCN conformers. Bars represent the rotational strength values of the lowest-energy conformer. 


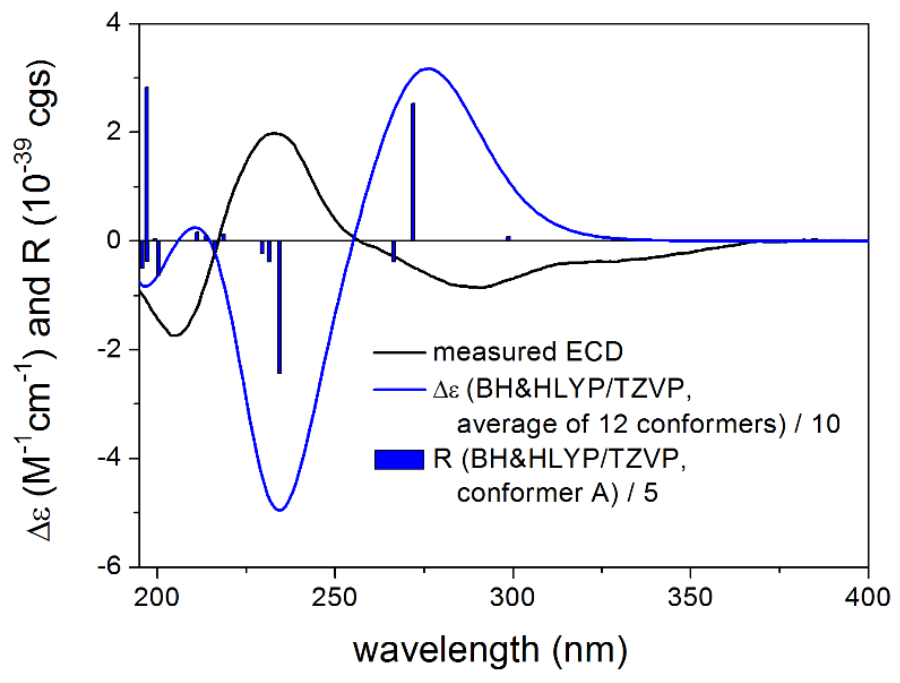

Figure S5. Experimental ECD spectrum of $\mathbf{1}$ in MeCN compared with the BH\&HLYP/TZVP PCM/MeCN ECD spectrum of $(3 S, 5 R, 9 S, 10 R, 13 S, 24 R)-1$ computed for the low-energy $(\geq 1 \%) \omega B 97 X / T Z V P$ PCM/MeCN conformers. Bars represent the rotational strength values of the lowest-energy conformer.

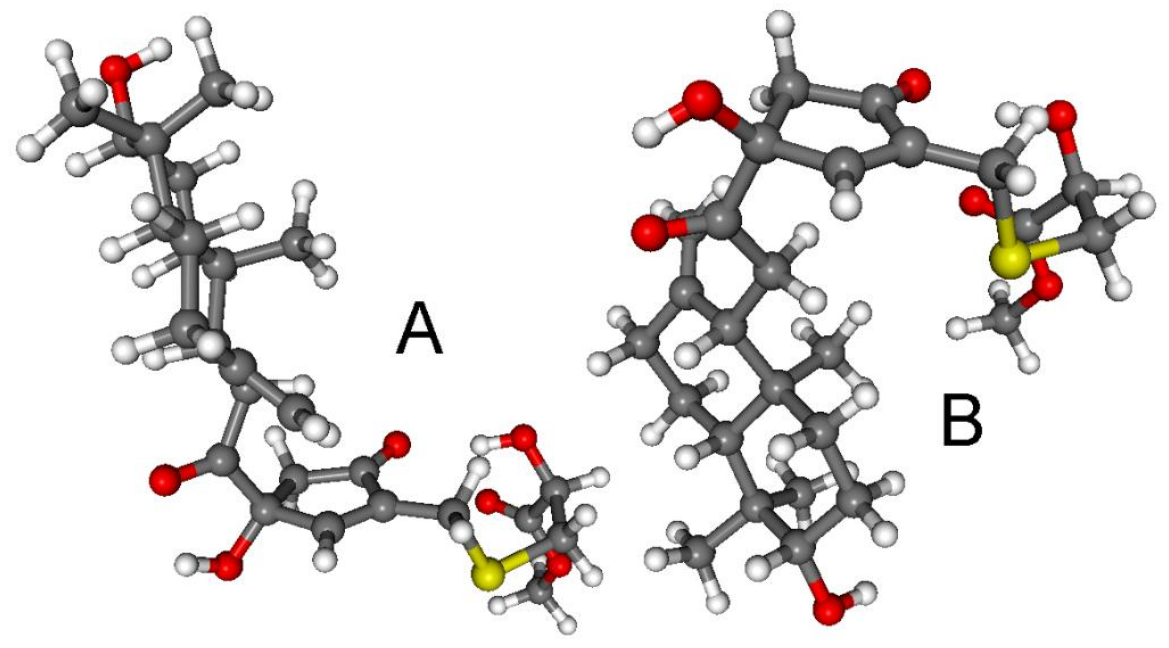

Figure S6. Structure and population of the lowest-energy $\omega \mathrm{B} 97 \mathrm{X} / \mathrm{TZVP}$ PCM/MeCN conformers of A: $(3 S, 5 R, 9 S, 10 R, 13 R, 24 R)-1$ and B: $(3 S, 5 R, 9 S, 10 R, 13 S, 24 R)-1$ with a Boltzmann-population of $16.1 \%$ and $30.1 \%$, respectively. 


\section{HRMS, IR, UV, [ $\alpha]_{D}$, ECD, and NMR Spectra of compound 1.}

\begin{tabular}{|c|c|c|c|}
\hline Data Filename & WHL-d13.d & Sample Name & \\
\hline Sample Type & Sample & Position & $\mathrm{P} 1-\mathrm{C7}$ \\
\hline Instrument Name & Instrument 1 & User Name & \\
\hline Acq Method & LCMS-TEST-POS-1U.m & Acquired Time & 10/31/2018 12:13:14 AM \\
\hline $\begin{array}{l}\text { IRM Calibration Status } \\
\text { Comment }\end{array}$ & Success & DA Method & $1 . \mathrm{m}$ \\
\hline
\end{tabular}

\section{User Spectra}

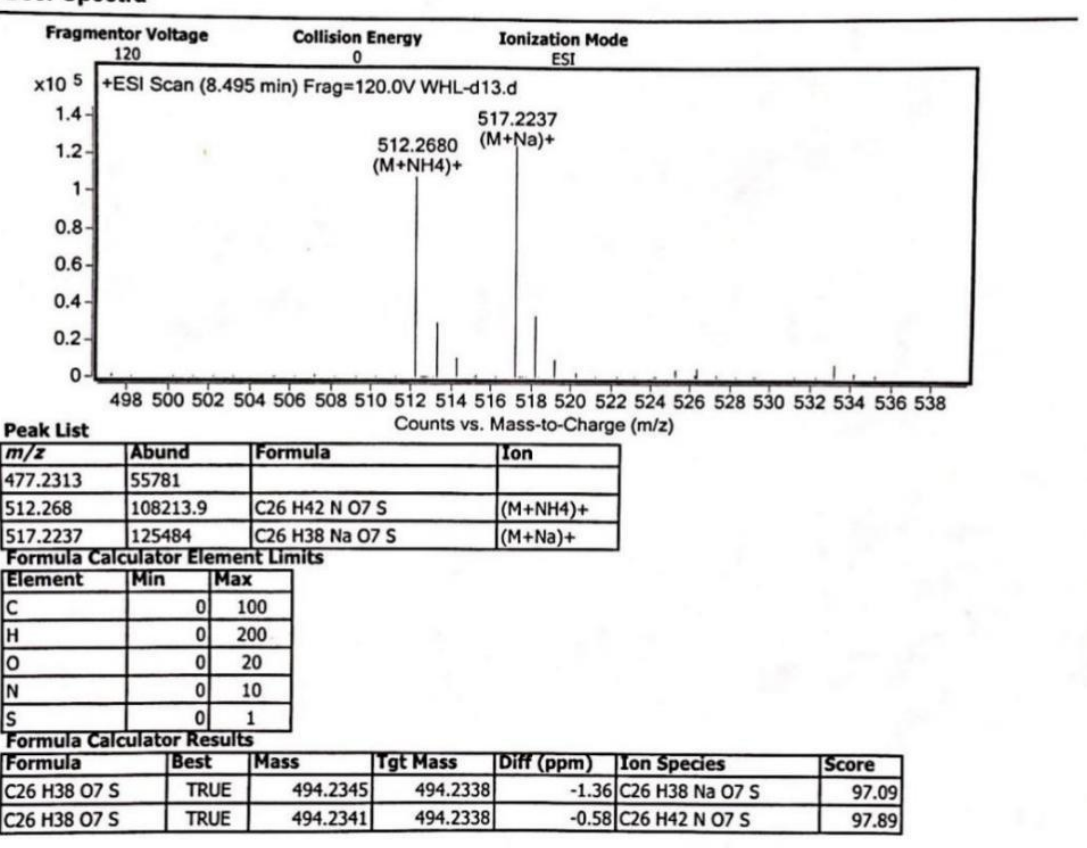

- End Of Report --

Figure S7. HRESI-MS spectrum of 1.

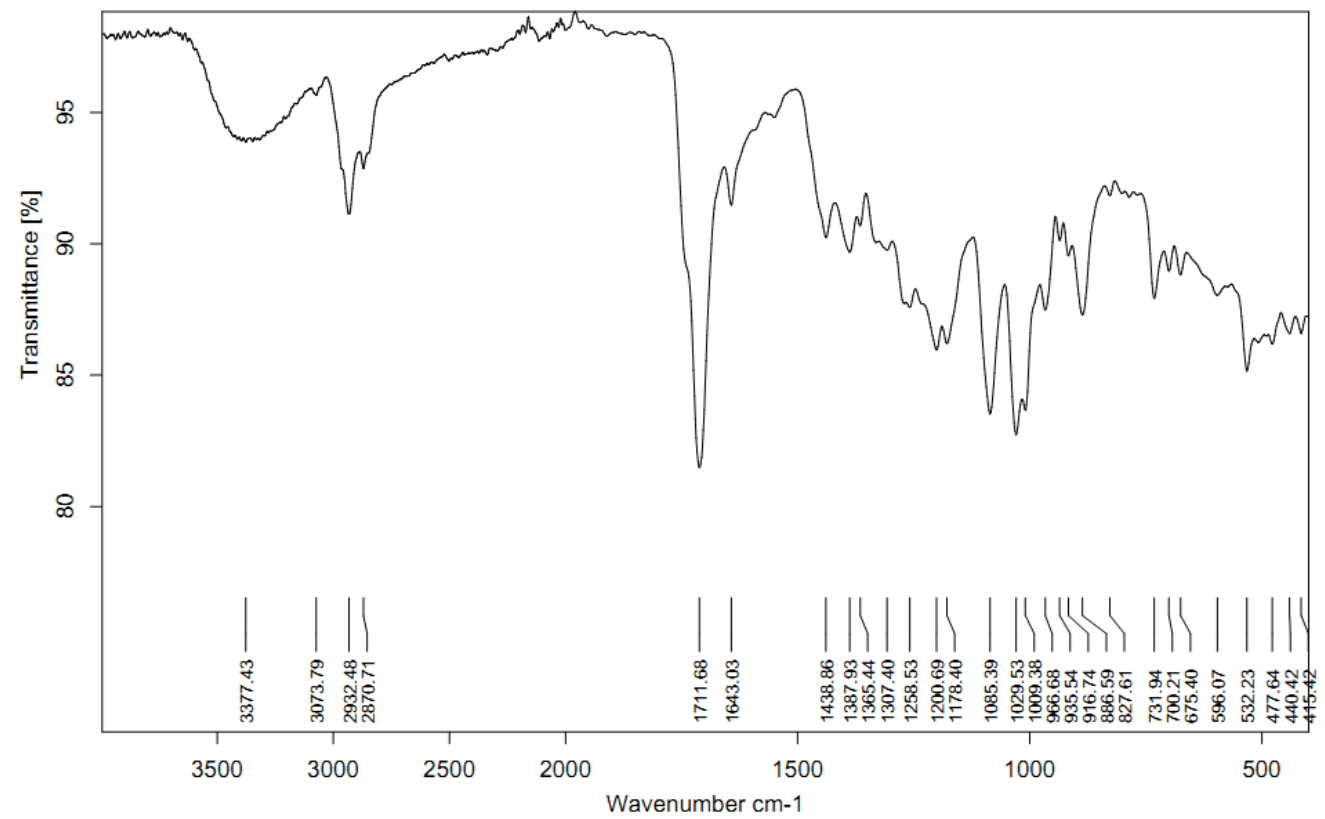

Figure S8. FTIR spectrum of 1. 


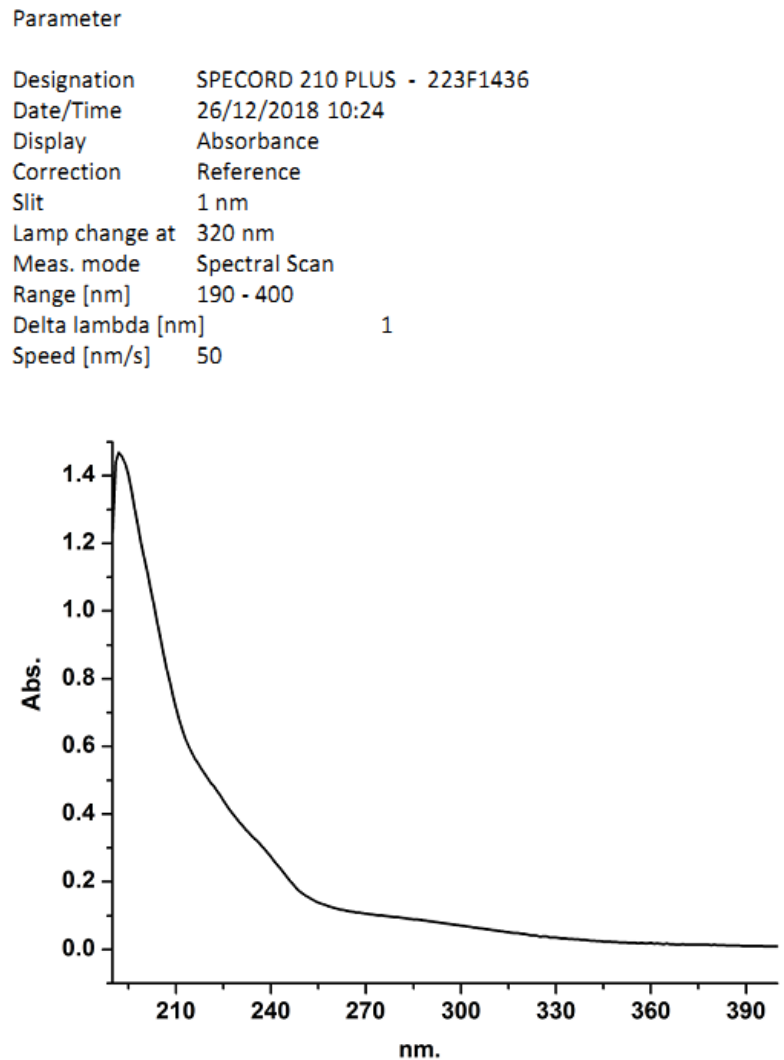

Figure S9. UV spectrum of 1 in MeCN.

\section{State Key Laboratory of Organometallic Chemistry}

This sample was measured on an Autopol VI, Serial \#91003

Manufactured by Rudolph Research Analytical, Hackettstown, NJ, USA.

Measurement Date : Thursday, 13-DEC-2018

Lot ID : whl-d13

Set Temperature : OFF

\begin{tabular}{lll} 
n & \multicolumn{2}{c}{ Average } \\
3 & \multicolumn{2}{c}{-117.78} \\
S.Ne & Time & Result \\
1 & $15: 08: 28$ & -117.78 \\
2 & $15: 08: 38$ & -118.15 \\
3 & $15: 08: 49$ & -117.41
\end{tabular}

\begin{tabular}{ll} 
Std.Dev. & \multicolumn{1}{c}{$\%$ RSD } \\
0.37 & \multicolumn{1}{c}{-0.31} \\
Scale & OR ${ }^{\circ}$ Arc \\
SR & -0.318 \\
SR & -0.319 \\
SR & -0.317
\end{tabular}

$\%$ RSD

Maximum

$-117.41$

Minimum

WLG.nm Lg.mm

$589 \quad 100.00$

$589 \quad 100.00$

$589 \quad 100.00$

\begin{tabular}{ll}
\multicolumn{2}{c}{$\begin{array}{c}\text { Minimum } \\
-118.15 \\
\text { Conc.g/100ml }\end{array}$} \\
\hline 0.270 & Temp. \\
0.270 & 22.8 \\
0.270 & 22.8 \\
& 22.8
\end{tabular}

File Name : C:Mutopol|DatalReadings_data_20181213150804.pdf

Figure S10. Optical rotation of 1 in $\mathrm{CHCl}_{3}$. 


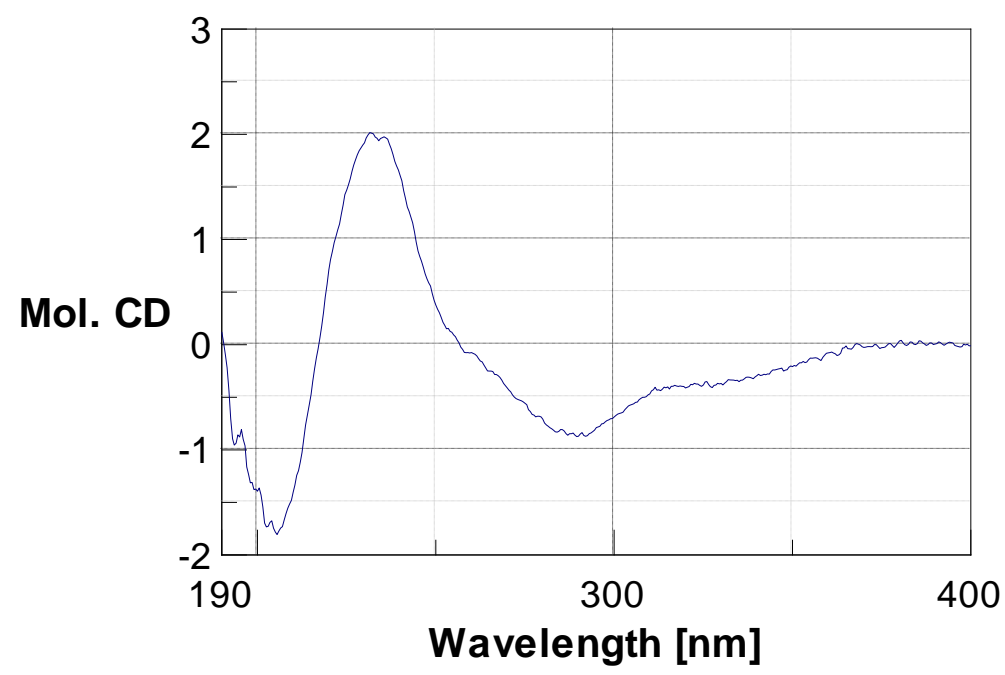

Figure S11. CD spectrum of $\mathbf{1}$ in MeCN.<smiles>COC(=O)[C@H](O)CSCC1=C2C(=CC1)C(=O)C[C@]1(O)C(=O)C[C@H]3C(=C21)CC[C@H]1C(C)(C)[C@@H](O)CC[C@]31C</smiles>

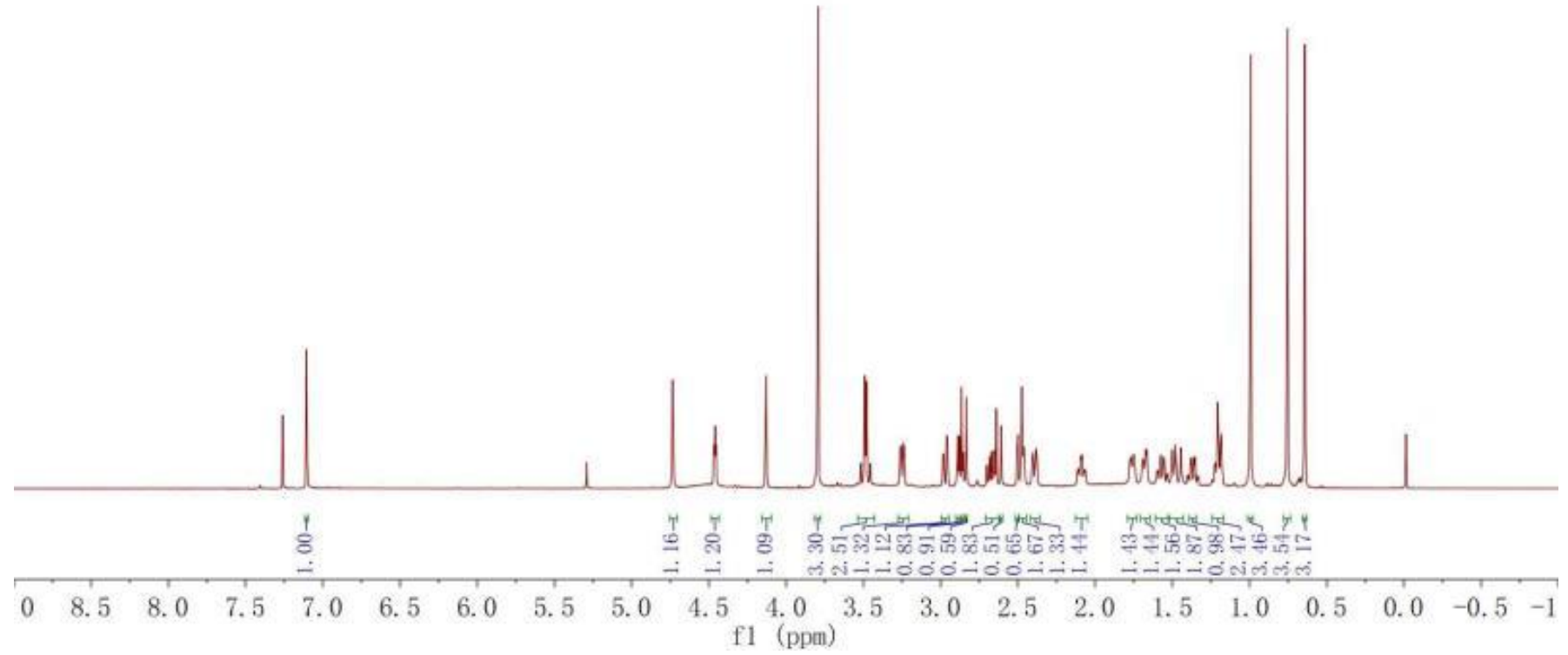

Figure S12. ${ }^{1} \mathrm{H}$ NMR (600 MHz, $\left.\mathrm{CDCl}_{3}\right)$ spectrum of $\mathbf{1}$. 


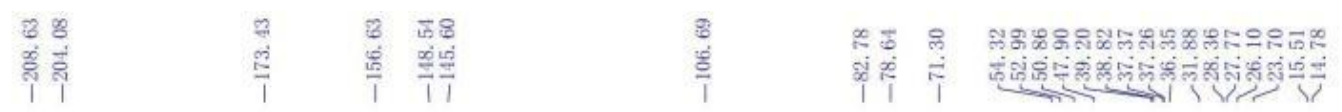<smiles>COC(=O)[C@H](O)CSCC1=C2C3=CC[C@H]4C(C)(C)[C@@H](O)CC[C@]4(C)[C@H]3CC(=O)[C@]2(O)CC1=O</smiles>
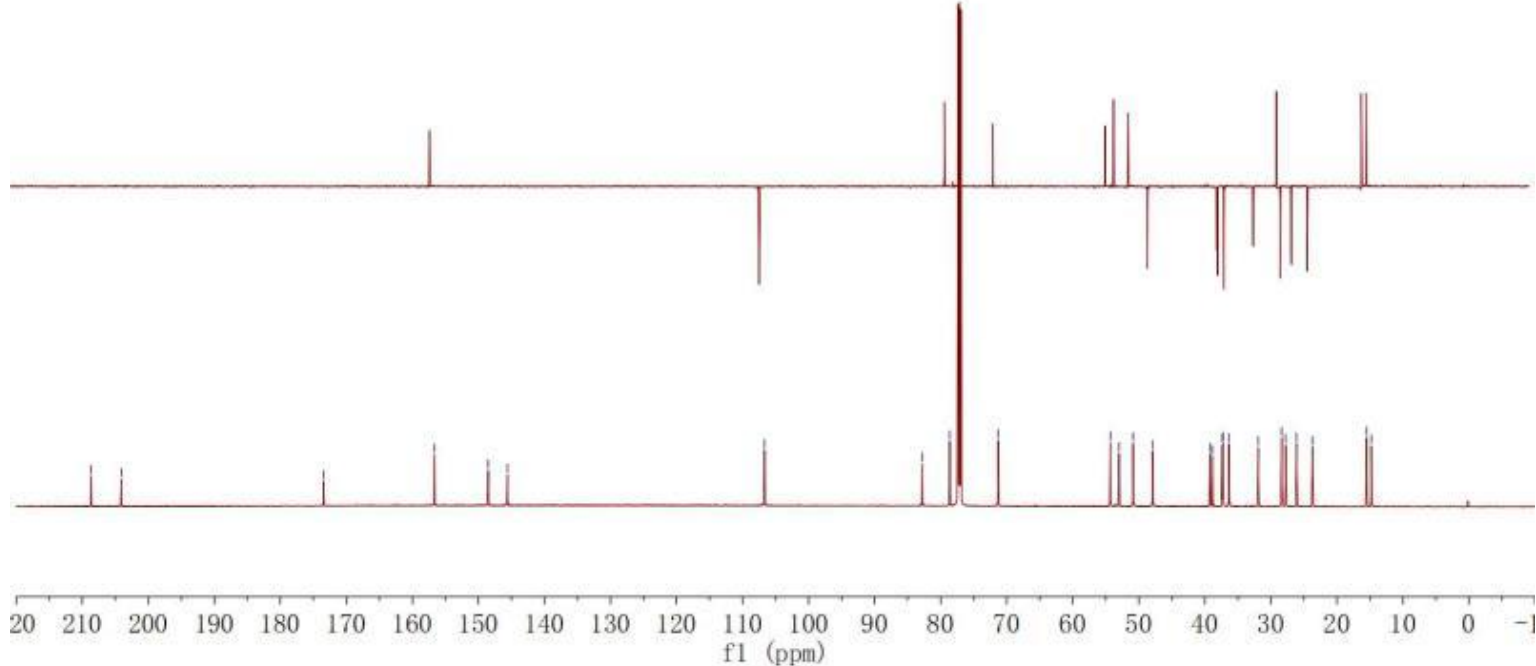

Figure S13. ${ }^{13} \mathrm{C}$ NMR and DEPT $\left(150 \mathrm{MHz}, \mathrm{CDCl}_{3}\right)$ spectra of $\mathbf{1}$.

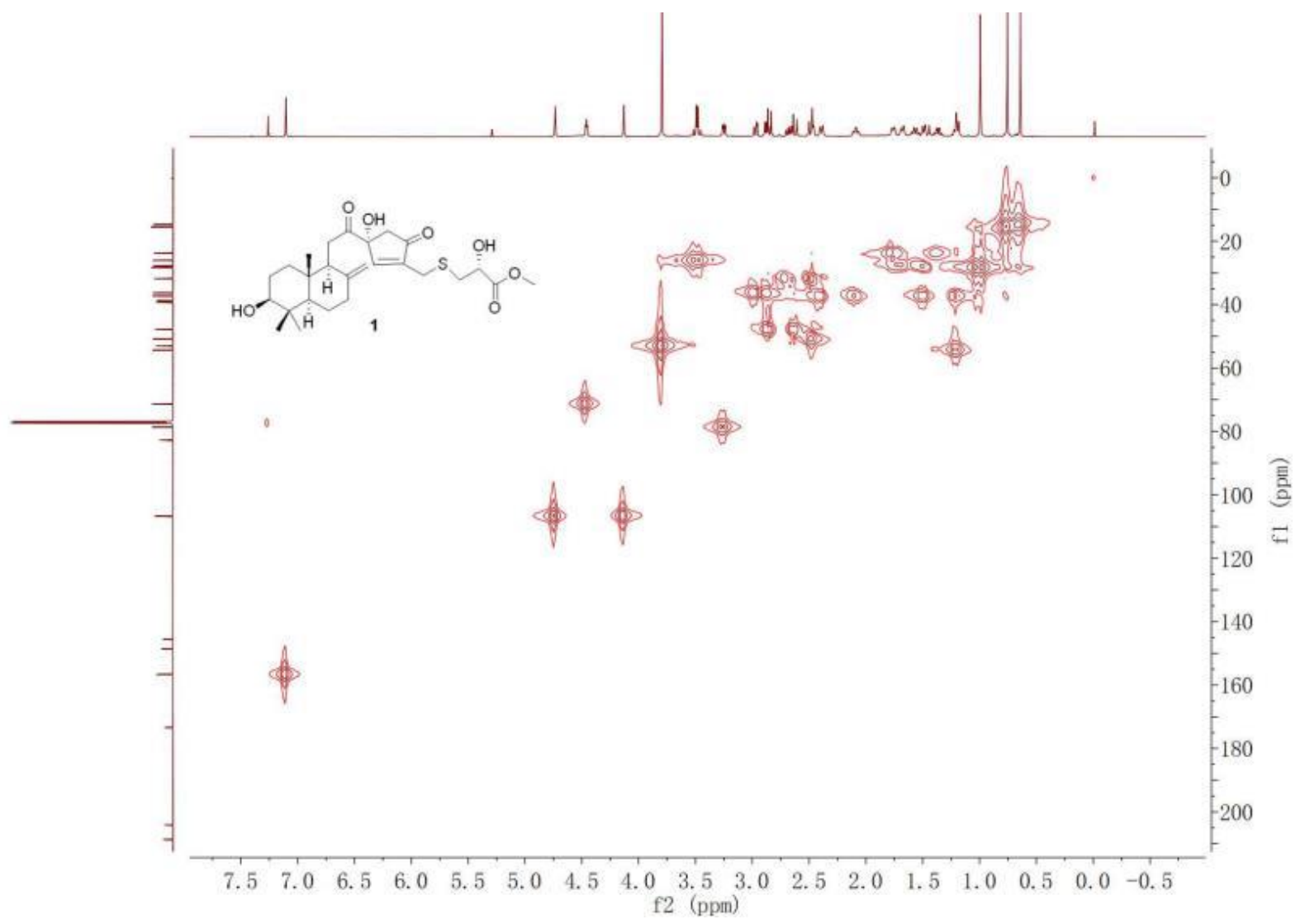

Figure S14. HSQC spectrum of 1 in $\mathrm{CDCl}_{3}$. 


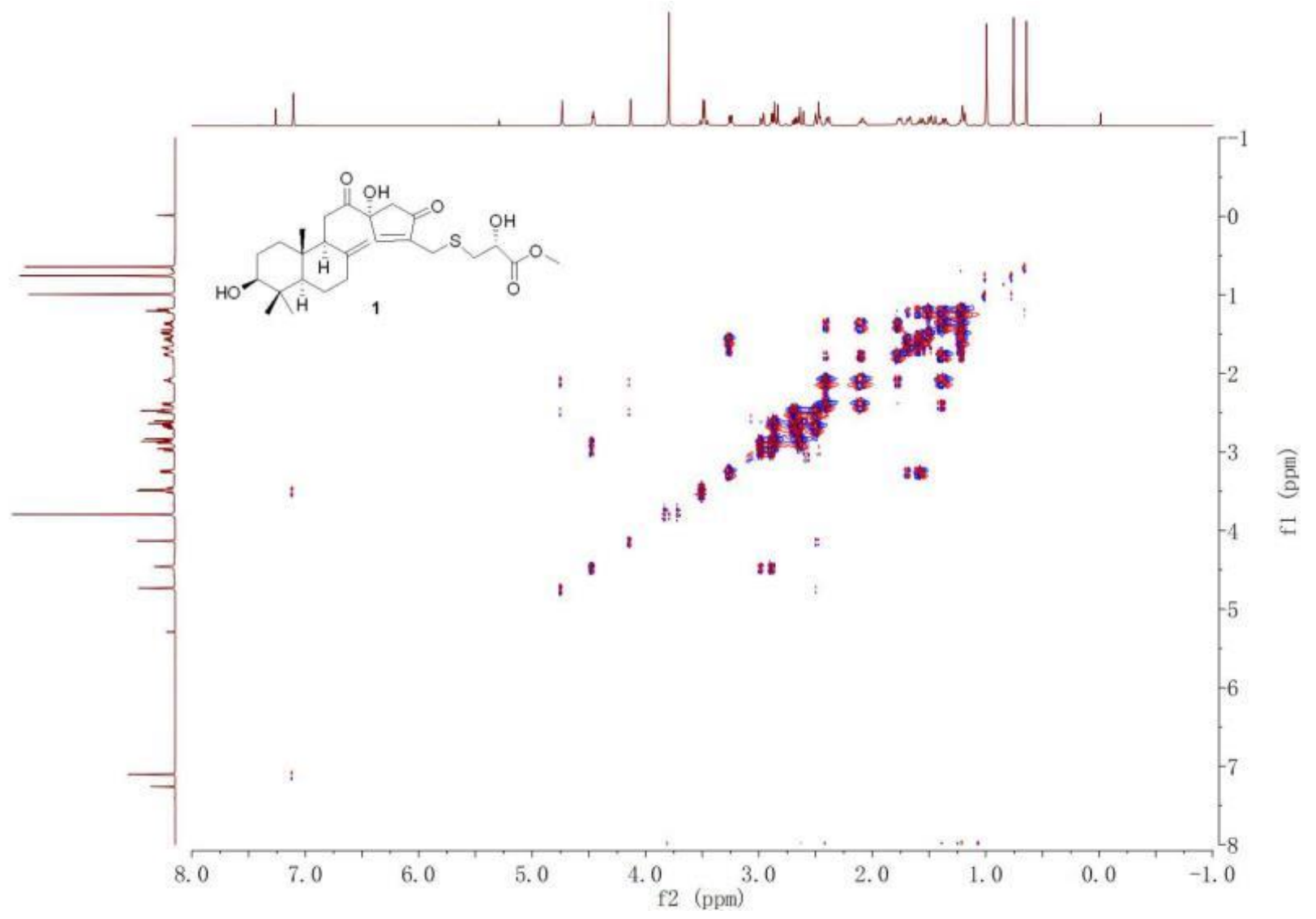

Figure S15. ${ }^{1} \mathrm{H}-{ }^{-1} \mathrm{H}$ COSY spectrum of 1 in $\mathrm{CDCl}_{3}$.

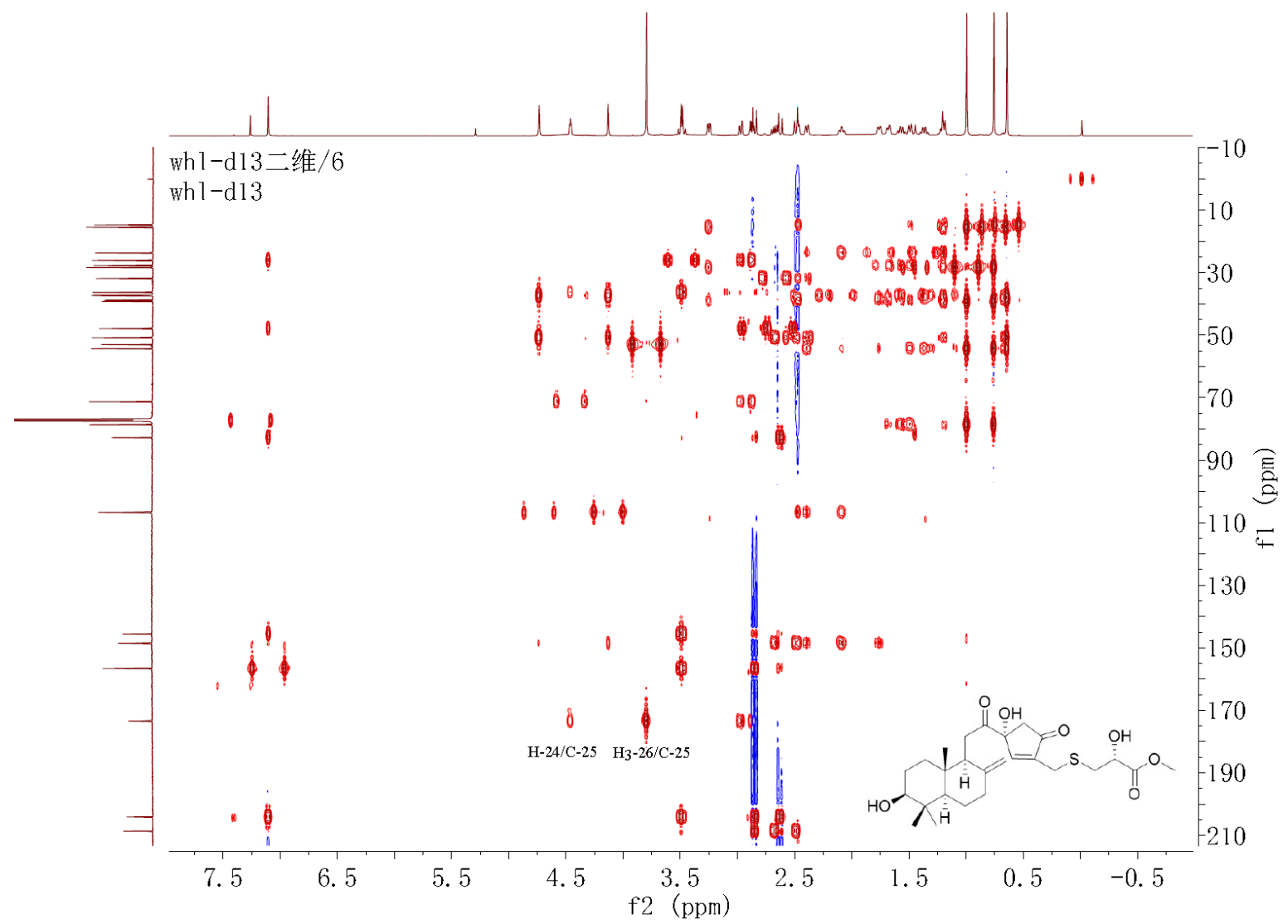

Figure S16. $\mathrm{HMBC}$ spectrum of 1 in $\mathrm{CDCl}_{3}$. 


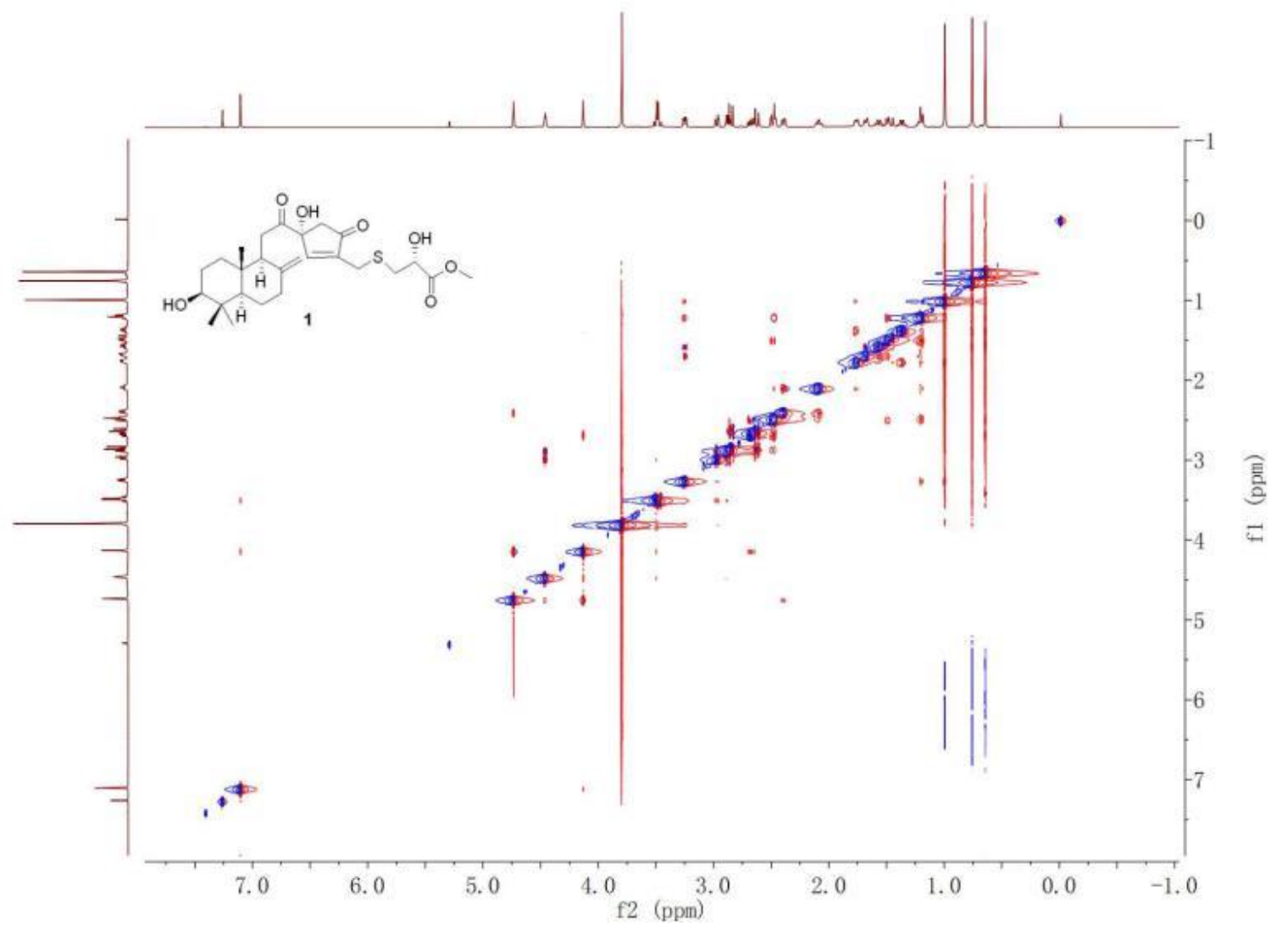

Figure S17. NOESY spectrum of $\mathbf{1}$ in $\mathrm{CDCl}_{3}$.

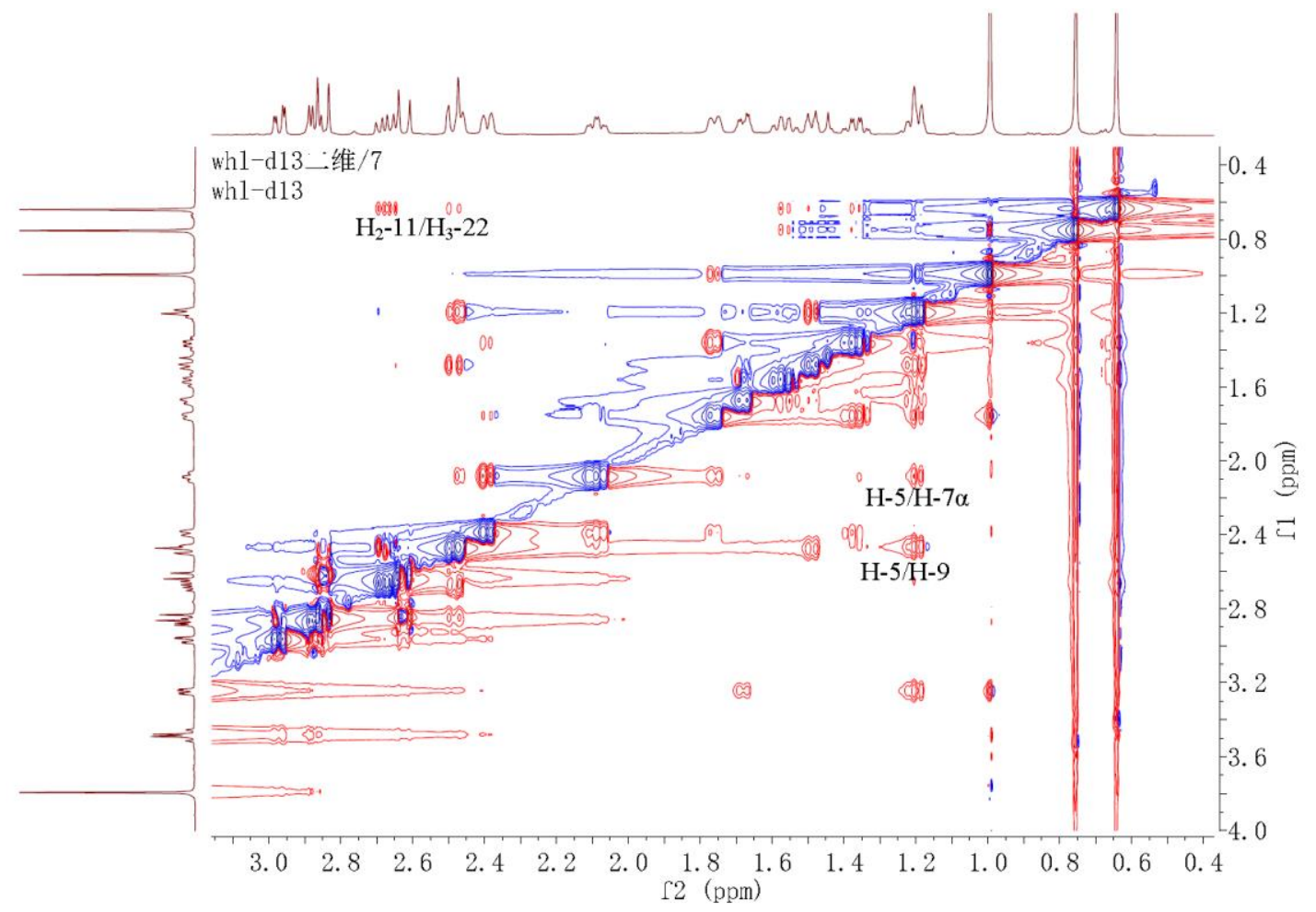

Figure S18. Partial enlarged NOESY spectrum of 1 in $\mathrm{CDCl}_{3}$. 


\section{Computational data for low-energy conformers of truncated model compound $(R)-4$ and the two epimers of compound 1.}

Table S1. Cartesian coordinates and energies of the low-energy conformers calculated for $(R)$-4-acetyl-4-hydroxy-2(methylthiomethyl)cyclopent-2-enone (4), $(3 S, 5 R, 9 S, 10 R, 13 R, 24 R)-1$ and $(3 S, 5 R, 9 S, 10 R, 13 S, 24 R)-1$ at the $\omega B$ 97X/TZVP PCM/MeCN level.

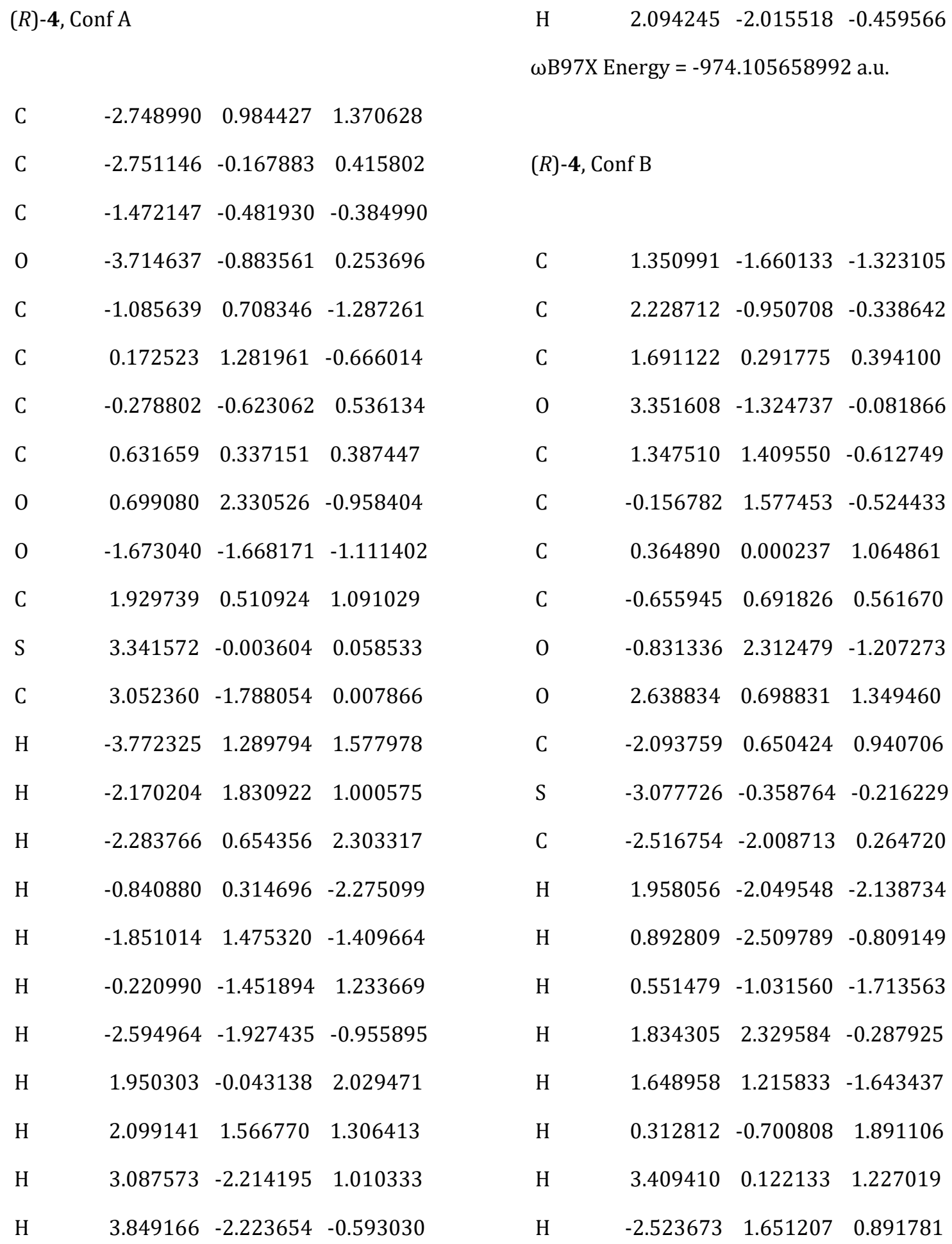




$\begin{array}{lllllllll}\mathrm{H} & -2.228679 & 0.264059 & 1.951062 & \mathrm{H} & 1.724829 & -1.888048 & 0.895806 \\ \mathrm{H} & -3.048228 & -2.723534 & -0.361588 & \mathrm{H} & 3.163871 & -1.207758 & 1.699587 \\ \mathrm{H} & -2.750577 & -2.205981 & 1.310631 & \mathrm{H} & 3.319609 & -2.607662 & 0.620048 \\ \mathrm{H} & -1.445816 & -2.124205 & 0.097120 & & & \end{array}$

$\omega$ B97X Energy $=-974.105604512$ a.u.

(R)-4, Conf D

(R)-4, Conf C

$\begin{array}{lllllllll} & & & & \mathrm{C} & -2.331602 & 0.012033 & 1.889794 \\ \mathrm{C} & -1.635542 & -1.212421 & 1.720360 & \mathrm{C} & -2.481638 & -0.632247 & 0.547291 \\ \mathrm{C} & -2.220015 & -0.978744 & 0.362213 & \mathrm{C} & -1.454321 & -0.313519 & -0.556190 \\ \mathrm{C} & -1.570221 & 0.062653 & -0.569115 & \mathrm{O} & -3.370392 & -1.414035 & 0.291058 \\ \mathrm{O} & -3.181178 & -1.590430 & -0.048642 & \mathrm{C} & -1.473071 & 1.190128 & -0.900120 \\ \mathrm{C} & -1.618507 & 1.467124 & 0.066232 & \mathrm{C} & -0.158721 & 1.729138 & -0.372477 \\ \mathrm{C} & -0.177378 & 1.796390 & 0.399605 & \mathrm{C} & -0.044092 & -0.541818 & -0.057105 \\ \mathrm{C} & -0.087908 & -0.202489 & -0.725495 & \mathrm{C} & 0.672965 & 0.572962 & 0.056168 \\ \mathrm{C} & 0.685926 & 0.742289 & -0.197996 & \mathrm{O} & 0.159950 & 2.894464 & -0.306687 \\ \mathrm{O} & 0.206605 & 2.745967 & 1.043480 & \mathrm{O} & -1.721200 & -1.126208 & -1.671236 \\ \mathrm{O} & -2.211305 & 0.012470 & -1.818919 & \mathrm{C} & 2.073357 & 0.778692 & 0.533946 \\ \mathrm{C} & 2.174261 & 0.863281 & -0.148413 & \mathrm{~S} & 3.052793 & -0.727519 & 0.758974 \\ \mathrm{~S} & 3.101157 & -0.611780 & -0.643378 & \mathrm{C} & 3.313216 & -1.190250 & -0.972183 \\ \mathrm{C} & 2.787611 & -1.672877 & 0.789093 & \mathrm{H} & -1.626949 & -0.584449 & 2.475714 \\ \mathrm{H} & -0.855130 & -1.972633 & 1.625625 & \mathrm{H} & -3.291463 & 0.020203 & 2.401961 \\ \mathrm{H} & -1.175877 & -0.319291 & 2.142804 & \mathrm{H} & -1.928453 & 1.022667 & 1.825887 \\ \mathrm{H} & -2.404920 & -1.592911 & 2.389281 & \mathrm{H} & -1.484977 & 1.288470 & -1.986584 \\ \mathrm{H} & -1.977731 & 2.169327 & -0.687334 & \mathrm{H} & -2.317433 & 1.753985 & -0.501759 \\ \mathrm{H} & -2.255018 & 1.563538 & 0.946709 & \mathrm{H} & 0.304071 & -1.543468 & 0.170102 \\ \mathrm{H} & 0.261355 & -1.093292 & -1.235990 & \mathrm{H} & -2.528467 & -1.620370 & -1.458278 \\ \mathrm{H} & -2.935396 & -0.626436 & -1.724876 & \mathrm{H} & 2.052438 & 1.278153 & 1.505243 \\ \mathrm{H} & 2.479531 & 1.165315 & 0.856276 & \mathrm{H} & 2.597647 & 1.452562 & -0.147244 \\ \mathrm{H} & 2.494019 & 1.663030 & -0.820142 & \mathrm{H} & 3.832685 & -0.394198 & -1.504808\end{array}$




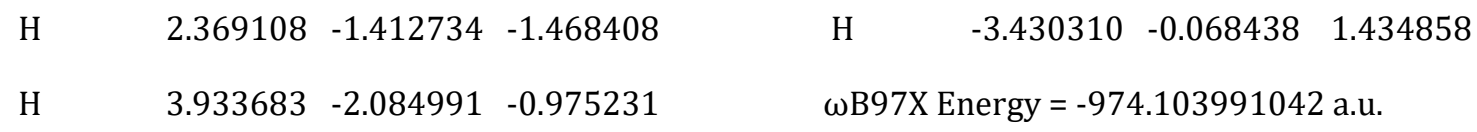

$\omega$ B97X Energy = -974.104331530 a.u.

(R)-4, Conf F

(R)-4, Conf E

\begin{tabular}{|c|c|c|c|c|c|c|c|}
\hline $\mathrm{C}$ & 1.299014 & 1.840179 & 1.042586 & $\mathrm{C}$ & -2.800461 & -0.163188 & 0.341017 \\
\hline $\mathrm{C}$ & 2.242858 & 0.998304 & 0.242250 & $\mathrm{C}$ & -1.516340 & -0.396585 & -0.479274 \\
\hline $\mathrm{C}$ & 1.743274 & -0.341866 & -0.333903 & 0 & -3.799311 & -0.767171 & 0.018509 \\
\hline 0 & 3.383766 & 1.329729 & 0.008781 & $\mathrm{C}$ & -1.030481 & 0.918395 & -1.122117 \\
\hline $\mathrm{C}$ & 1.311028 & -1.299460 & 0.794949 & $\mathrm{C}$ & 0.247305 & 1.268806 & -0.385919 \\
\hline C & -0.196830 & -1.398682 & 0.674400 & $\mathrm{C}$ & -0.366014 & -0.790818 & 0.421775 \\
\hline $\mathrm{C}$ & 0.469808 & -0.139265 & -1.125628 & C & 0.606641 & 0.116647 & 0.482851 \\
\hline $\mathrm{C}$ & -0.604387 & -0.698973 & -0.572597 & 0 & 0.863022 & 2.305834 & -0.482116 \\
\hline 0 & -0.937186 & -1.954928 & 1.453062 & 0 & -1.763398 & -1.402881 & -1.428714 \\
\hline 0 & 2.748970 & -0.887434 & -1.149795 & $\mathrm{C}$ & 1.876951 & 0.080517 & 1.263880 \\
\hline $\mathrm{C}$ & -2.017065 & -0.682533 & -1.053655 & S & 3.184663 & -0.932133 & 0.499640 \\
\hline S & -2.892954 & 0.880511 & -0.724121 & $\mathrm{C}$ & 3.421454 & -0.036545 & -1.054804 \\
\hline $\mathrm{C}$ & -2.953145 & 0.845782 & 1.084068 & $\mathrm{H}$ & -2.122437 & 1.638546 & 1.322083 \\
\hline $\mathrm{H}$ & 1.856994 & 2.581159 & 1.610552 & $\mathrm{H}$ & -2.341964 & 0.238042 & 2.363099 \\
\hline $\mathrm{H}$ & 0.622169 & 2.353625 & 0.353769 & $\mathrm{H}$ & -3.769654 & 1.096380 & 1.752831 \\
\hline $\mathrm{H}$ & 0.683938 & 1.239082 & 1.713679 & $\mathrm{H}$ & -0.792404 & 0.716120 & -2.167474 \\
\hline $\mathrm{H}$ & 1.747853 & -2.278774 & 0.595105 & $\mathrm{H}$ & -1.739699 & 1.746362 & -1.093055 \\
\hline $\mathrm{H}$ & 1.603372 & -1.001236 & 1.802318 & $\mathrm{H}$ & -0.380936 & -1.742531 & 0.942041 \\
\hline $\mathrm{H}$ & 0.487448 & 0.420120 & -2.054917 & $\mathrm{H}$ & -2.703796 & -1.627045 & -1.345664 \\
\hline $\mathrm{H}$ & 3.514863 & -0.298011 & -1.066600 & $\mathrm{H}$ & 1.712803 & -0.359226 & 2.247216 \\
\hline $\mathrm{H}$ & -2.574663 & -1.499996 & -0.594889 & $\mathrm{H}$ & 2.252034 & 1.096068 & 1.399141 \\
\hline $\mathrm{H}$ & -2.053650 & -0.808859 & -2.135566 & $\mathrm{H}$ & 4.264021 & -0.501260 & -1.564382 \\
\hline $\mathrm{H}$ & -3.545741 & 1.703409 & 1.398887 & $\mathrm{H}$ & 3.651222 & 1.011278 & -0.864019 \\
\hline $\mathrm{H}$ & -1.956753 & 0.929881 & 1.517544 & $\mathrm{H}$ & 2.540726 & -0.111842 & -1.692644 \\
\hline
\end{tabular}


$\omega B$ 97X Energy $=-974.103690287$ a.u.

(R)-4, Conf $\mathrm{H}$

(R)-4, Conf G

\begin{tabular}{|c|c|c|c|c|c|c|c|}
\hline $\mathrm{C}$ & 1.556597 & -1.764402 & -1.192774 & $\mathrm{C}$ & -2.874015 & -0.094609 & 0.532946 \\
\hline $\mathrm{C}$ & 2.426138 & -0.977016 & -0.263806 & $\mathrm{C}$ & -1.701440 & -0.453296 & -0.401197 \\
\hline $\mathrm{C}$ & 1.851470 & 0.284854 & 0.410547 & 0 & -3.919419 & -0.688476 & 0.388742 \\
\hline 0 & 3.564313 & -1.295101 & 0.000023 & $\mathrm{C}$ & -1.265581 & 0.777694 & -1.222860 \\
\hline $\mathrm{C}$ & 1.482094 & 1.347075 & -0.646095 & $\mathrm{C}$ & 0.088885 & 1.164449 & -0.663843 \\
\hline $\mathrm{C}$ & -0.031960 & 1.420183 & -0.622974 & $\mathrm{C}$ & -0.461109 & -0.799694 & 0.394661 \\
\hline $\mathrm{C}$ & 0.528447 & -0.020113 & 1.078527 & $\mathrm{C}$ & 0.526685 & 0.082380 & 0.258142 \\
\hline $\mathrm{C}$ & -0.512491 & 0.582028 & 0.507580 & 0 & 0.698983 & 2.179486 & -0.907951 \\
\hline 0 & -0.725985 & 2.057643 & -1.380537 & 0 & -2.082147 & -1.537270 & -1.209845 \\
\hline 0 & 2.781018 & 0.758079 & 1.352161 & $\mathrm{C}$ & 1.881656 & 0.070665 & 0.873710 \\
\hline $\mathrm{C}$ & -1.956606 & 0.469535 & 0.851602 & $S$ & 3.071471 & -0.660951 & -0.301524 \\
\hline$S$ & -2.699075 & -0.867534 & -0.145231 & $\mathrm{C}$ & 4.610824 & -0.256223 & 0.558188 \\
\hline $\mathrm{C}$ & -4.443955 & -0.576619 & 0.234399 & $\mathrm{H}$ & -2.010827 & 1.750977 & 1.257847 \\
\hline $\mathrm{H}$ & 2.174441 & -2.386744 & -1.836485 & $\mathrm{H}$ & -2.206248 & 0.474061 & 2.452470 \\
\hline $\mathrm{H}$ & 0.911110 & -2.410079 & -0.590668 & $\mathrm{H}$ & -3.635960 & 1.356925 & 1.887438 \\
\hline $\mathrm{H}$ & 0.906667 & -1.127403 & -1.793358 & $\mathrm{H}$ & -1.140647 & 0.462591 & -2.259954 \\
\hline $\mathrm{H}$ & 1.890157 & 2.305121 & -0.321410 & $\mathrm{H}$ & -1.955047 & 1.622267 & -1.206641 \\
\hline $\mathrm{H}$ & 1.846355 & 1.151405 & -1.655390 & $\mathrm{H}$ & -0.432283 & -1.698930 & 1.000298 \\
\hline $\mathrm{H}$ & 0.488866 & -0.676376 & 1.941280 & $\mathrm{H}$ & -3.012801 & -1.715880 & -1.001982 \\
\hline $\mathrm{H}$ & 3.574286 & 0.209473 & 1.247483 & $\mathrm{H}$ & 1.885516 & -0.500781 & 1.801505 \\
\hline $\mathrm{H}$ & -2.467186 & 1.402375 & 0.607170 & $\mathrm{H}$ & 2.194280 & 1.096225 & 1.079608 \\
\hline $\mathrm{H}$ & -2.091171 & 0.251746 & 1.910912 & $\mathrm{H}$ & 4.640363 & -0.724716 & 1.541371 \\
\hline $\mathrm{H}$ & -5.020829 & -1.325304 & -0.306330 & $\mathrm{H}$ & 4.725951 & 0.822723 & 0.656805 \\
\hline $\mathrm{H}$ & -4.749833 & 0.415313 & -0.096698 & $\mathrm{H}$ & 5.428793 & -0.647141 & -0.044957 \\
\hline $\mathrm{H}$ & -4.630820 & -0.687101 & 1.301985 & & $r g y=-c$ & 4.1033804 & 58 a.u. \\
\hline
\end{tabular}

$\omega$ B97X Energy $=-974.103570262$ a.u. 
(R)-4, Conf I

$(3 S, 5 R, 9 S, 10 R, 13 R, 24 R)-\mathbf{1}$, Conf A

\begin{tabular}{|c|c|c|c|c|c|c|c|}
\hline $\mathrm{C}$ & -1.998617 & -0.719915 & 1.949114 & & & & \\
\hline $\mathrm{C}$ & -2.481241 & -0.818154 & 0.535922 & $\mathrm{C}$ & 4.665250 & -1.996654 & -1.592548 \\
\hline $\mathrm{C}$ & -1.696024 & -0.096206 & -0.577115 & $\mathrm{C}$ & 6.024564 & -1.486211 & -1.147716 \\
\hline 0 & -3.458230 & -1.459847 & 0.219346 & $\mathrm{C}$ & 6.032961 & -0.958559 & 0.304868 \\
\hline $\mathrm{C}$ & -1.682852 & 1.426973 & -0.336668 & $\mathrm{C}$ & 4.879434 & 0.081422 & 0.441561 \\
\hline $\mathrm{C}$ & -0.254921 & 1.750832 & 0.051543 & $\mathrm{C}$ & 3.459385 & -0.360978 & -0.037033 \\
\hline $\mathrm{C}$ & -0.230292 & -0.472411 & -0.525178 & C & 3.588659 & -0.926872 & -1.461211 \\
\hline $\mathrm{C}$ & 0.568270 & 0.532216 & -0.172193 & C & 4.827808 & 0.747063 & 1.819769 \\
\hline 0 & 0.149204 & 2.809478 & 0.474265 & C & 3.890072 & 1.954933 & 1.821785 \\
\hline 0 & -2.251075 & -0.442936 & -1.820235 & C & 2.532449 & 1.578839 & 1.297093 \\
\hline $\mathrm{C}$ & 2.044433 & 0.580220 & 0.019046 & C & 2.586138 & 0.939804 & -0.075194 \\
\hline S & 2.818358 & -1.053132 & -0.134483 & C & 2.783647 & -1.399297 & 0.867883 \\
\hline $\mathrm{C}$ & 4.535818 & -0.565462 & 0.155396 & $\mathrm{H}$ & 5.161678 & 0.875975 & -0.265878 \\
\hline $\mathrm{H}$ & -1.571833 & 0.256917 & 2.177196 & $\mathrm{C}$ & 5.968493 & -2.118506 & 1.308131 \\
\hline $\mathrm{H}$ & -2.814833 & -0.944130 & 2.632522 & C & 7.375687 & -0.242547 & 0.519752 \\
\hline $\mathrm{H}$ & -1.207743 & -1.461688 & 2.090471 & 0 & 7.034906 & -2.468550 & -1.377161 \\
\hline $\mathrm{H}$ & -1.910709 & 1.919012 & -1.283372 & $\mathrm{C}$ & 1.210782 & 0.758251 & -0.715030 \\
\hline $\mathrm{H}$ & -2.386926 & 1.792330 & 0.411705 & C & 0.581363 & 2.055178 & -1.153747 \\
\hline $\mathrm{H}$ & 0.078133 & -1.484647 & -0.763215 & $\mathrm{C}$ & -0.954486 & 2.135786 & -1.317060 \\
\hline $\mathrm{H}$ & -3.028913 & -0.989264 & -1.625991 & 0 & 1.223125 & 3.043844 & -1.427880 \\
\hline $\mathrm{H}$ & 2.258268 & 0.995327 & 1.007231 & C & -1.553893 & 0.907273 & -2.022371 \\
\hline $\mathrm{H}$ & 2.481512 & 1.259876 & -0.717033 & $\mathrm{C}$ & -2.302496 & 0.158615 & -0.943261 \\
\hline $\mathrm{H}$ & 4.650057 & -0.122296 & 1.144108 & C & -1.615392 & 2.106819 & 0.048647 \\
\hline $\mathrm{H}$ & 5.139996 & -1.469315 & 0.099066 & $\mathrm{C}$ & -2.356615 & 1.020980 & 0.263669 \\
\hline $\mathrm{H}$ & 4.872608 & 0.135871 & -0.607171 & C & 1.430189 & 1.765820 & 2.014270 \\
\hline \multirow{3}{*}{\multicolumn{4}{|c|}{$\omega B 97 X$ Energy = -974.102917942 a.u. }} & 0 & -2.769668 & -0.956077 & -1.049633 \\
\hline & & & & 0 & -1.271646 & 3.328231 & -1.990148 \\
\hline & & & & C & -3.142769 & 0.653333 & 1.472896 \\
\hline
\end{tabular}




\begin{tabular}{|c|c|c|c|c|c|c|c|}
\hline S & -4.933972 & 0.886585 & 1.152757 & $\mathrm{H}$ & -2.278601 & 1.263007 & -2.757092 \\
\hline $\mathrm{C}$ & -5.656293 & -0.573738 & 1.958995 & $\mathrm{H}$ & -0.845594 & 0.259337 & -2.537068 \\
\hline $\mathrm{C}$ & -5.673700 & -1.837766 & 1.096899 & $\mathrm{H}$ & -1.474050 & 2.928258 & 0.742843 \\
\hline $\mathrm{C}$ & -6.465452 & -1.616414 & -0.188829 & $\mathrm{H}$ & 0.443708 & 1.474909 & 1.671889 \\
\hline 0 & -4.399291 & -2.348182 & 0.822414 & $\mathrm{H}$ & 1.482172 & 2.220952 & 2.997714 \\
\hline 0 & -7.703725 & -1.204596 & 0.071130 & $\mathrm{H}$ & -0.457783 & 3.855831 & -2.005634 \\
\hline 0 & -6.028996 & -1.793189 & -1.297562 & $\mathrm{H}$ & -2.846221 & 1.261412 & 2.325800 \\
\hline C & -8.548623 & -0.963591 & -1.065194 & $\mathrm{H}$ & -2.980799 & -0.397377 & 1.712619 \\
\hline $\mathrm{H}$ & 3.130329 & 1.641588 & -0.718241 & $\mathrm{H}$ & -5.118242 & -0.775702 & 2.884908 \\
\hline $\mathrm{H}$ & 4.395600 & -2.886535 & -1.013097 & $\mathrm{H}$ & -6.677736 & -0.296628 & 2.218140 \\
\hline $\mathrm{H}$ & 4.739511 & -2.318676 & -2.634166 & $\mathrm{H}$ & -6.206865 & -2.606101 & 1.665794 \\
\hline $\mathrm{H}$ & 6.305960 & -0.649582 & -1.797548 & $\mathrm{H}$ & -3.976573 & -1.834794 & 0.110866 \\
\hline $\mathrm{H}$ & 3.822226 & -0.105212 & -2.149851 & $\mathrm{H}$ & -8.687511 & -1.884075 & -1.631126 \\
\hline $\mathrm{H}$ & 2.636926 & -1.352737 & -1.789134 & $\mathrm{H}$ & -9.495260 & -0.620539 & -0.658691 \\
\hline $\mathrm{H}$ & 5.824725 & 1.077229 & 2.115479 & $\mathrm{H}$ & -8.109025 & -0.200141 & -1.706007 \\
\hline $\mathrm{H}$ & 4.497108 & 0.034020 & 2.580176 & \multicolumn{4}{|c|}{$\omega \mathrm{B} 97 \mathrm{X}$ Energy = -1938.58995275 a.u. } \\
\hline $\mathrm{H}$ & 3.806651 & 2.376755 & 2.825573 & & & & \\
\hline $\mathrm{H}$ & 4.316022 & 2.731428 & 1.174837 & \multicolumn{4}{|c|}{$(3 S, 5 R, 9 S, 10 R, 13 R, 24 R)-\mathbf{1}$, Conf B } \\
\hline $\mathrm{H}$ & 1.745425 & -1.548559 & 0.560564 & & & & \\
\hline $\mathrm{H}$ & 3.268856 & -2.371519 & 0.810764 & $\mathrm{C}$ & -3.673065 & -1.751321 & -1.811541 \\
\hline $\mathrm{H}$ & 2.771286 & -1.093827 & 1.914593 & $\mathrm{C}$ & -4.897996 & -1.328222 & -1.020629 \\
\hline $\mathrm{H}$ & 5.189744 & -2.847725 & 1.084980 & $\mathrm{C}$ & -4.700410 & 0.004359 & -0.263285 \\
\hline $\mathrm{H}$ & 5.794462 & -1.749531 & 2.320395 & $\mathrm{C}$ & -3.392938 & -0.109567 & 0.581623 \\
\hline $\mathrm{H}$ & 6.923243 & -2.649975 & 1.323389 & $\mathrm{C}$ & -2.104248 & -0.605364 & -0.147509 \\
\hline $\mathrm{H}$ & 8.190539 & -0.885659 & 0.183049 & $\mathrm{C}$ & -2.450874 & -1.892626 & -0.914045 \\
\hline $\mathrm{H}$ & 7.550941 & -0.013225 & 1.572291 & $\mathrm{C}$ & -3.107748 & 1.137950 & 1.420120 \\
\hline $\mathrm{H}$ & 7.421676 & 0.692786 & -0.045699 & $\mathrm{C}$ & -2.018508 & 0.867762 & 2.456672 \\
\hline $\mathrm{H}$ & 6.775505 & -3.278489 & -0.928034 & $\mathrm{C}$ & -0.783029 & 0.288532 & 1.823125 \\
\hline $\mathrm{H}$ & 1.303570 & 0.175198 & -1.636433 & $\mathrm{C}$ & -1.057351 & -0.937353 & 0.974153 \\
\hline $\mathrm{H}$ & 0.519322 & 0.202804 & -0.078507 & $\mathrm{C}$ & -1.499657 & 0.420724 & -1.115815 \\
\hline
\end{tabular}




\begin{tabular}{|c|c|c|c|c|c|c|c|}
\hline $\mathrm{H}$ & -3.618016 & -0.911937 & 1.300344 & $\mathrm{H}$ & -4.011098 & 1.462893 & 1.938140 \\
\hline $\mathrm{C}$ & -4.734529 & 1.192111 & -1.236088 & $\mathrm{H}$ & -2.801345 & 1.968963 & 0.777919 \\
\hline $\mathrm{C}$ & -5.891525 & 0.163307 & 0.694542 & $\mathrm{H}$ & -1.769583 & 1.780115 & 3.003557 \\
\hline 0 & -6.060611 & -1.316730 & -1.848978 & $\mathrm{H}$ & -2.404225 & 0.147566 & 3.188590 \\
\hline $\mathrm{C}$ & 0.203158 & -1.617115 & 0.440869 & $\mathrm{H}$ & -2.183508 & 0.679749 & -1.920767 \\
\hline $\mathrm{C}$ & 1.083213 & -2.227117 & 1.499442 & $\mathrm{H}$ & -1.209973 & 1.345909 & -0.614445 \\
\hline $\mathrm{C}$ & 2.567882 & -2.510645 & 1.164372 & $\mathrm{H}$ & -0.601623 & 0.011744 & -1.586639 \\
\hline 0 & 0.678239 & -2.559420 & 2.590292 & $\mathrm{H}$ & -4.116558 & 1.049282 & -2.122864 \\
\hline $\mathrm{C}$ & 2.717576 & -3.358988 & -0.115632 & $\mathrm{H}$ & -4.403217 & 2.110179 & -0.747712 \\
\hline $\mathrm{C}$ & 3.422682 & -2.463055 & -1.116004 & $\mathrm{H}$ & -5.758836 & 1.363684 & -1.575671 \\
\hline $\mathrm{C}$ & 3.314134 & -1.234023 & 0.823025 & $\mathrm{H}$ & -6.823859 & 0.009643 & 0.148564 \\
\hline $\mathrm{C}$ & 3.770604 & -1.190003 & -0.427825 & $\mathrm{H}$ & -5.930419 & 1.160314 & 1.136714 \\
\hline $\mathrm{C}$ & 0.409339 & 0.841402 & 2.012165 & $\mathrm{H}$ & -5.846802 & -0.568631 & 1.506165 \\
\hline 0 & 3.682759 & -2.746942 & -2.261624 & $\mathrm{H}$ & -5.886045 & -0.750001 & -2.606154 \\
\hline 0 & 3.163537 & -3.128723 & 2.278664 & $\mathrm{H}$ & -0.081147 & -2.456715 & -0.200395 \\
\hline $\mathrm{C}$ & 4.527649 & -0.099969 & -1.100702 & $\mathrm{H}$ & 0.798613 & -0.956187 & -0.190905 \\
\hline$S$ & 3.510004 & 0.812259 & -2.315930 & $\mathrm{H}$ & 3.358073 & -4.211979 & 0.111748 \\
\hline $\mathrm{C}$ & 2.283437 & 1.621026 & -1.257147 & $\mathrm{H}$ & 1.788732 & -3.743804 & -0.536669 \\
\hline $\mathrm{C}$ & 2.761668 & 2.894391 & -0.574428 & $\mathrm{H}$ & 3.471169 & -0.471347 & 1.578805 \\
\hline $\mathrm{C}$ & 1.646550 & 3.534400 & 0.246769 & $\mathrm{H}$ & 1.319974 & 0.445155 & 1.578397 \\
\hline 0 & 3.854033 & 2.627798 & 0.274086 & $\mathrm{H}$ & 0.525317 & 1.724942 & 2.631053 \\
\hline 0 & 0.509746 & 3.644407 & -0.417153 & $\mathrm{H}$ & 2.469804 & -3.195922 & 2.953819 \\
\hline 0 & 1.821218 & 3.908040 & 1.383068 & $\mathrm{H}$ & 5.341997 & -0.519135 & -1.693321 \\
\hline $\mathrm{C}$ & -0.593555 & 4.235936 & 0.292212 & $\mathrm{H}$ & 4.934075 & 0.604206 & -0.379158 \\
\hline $\mathrm{H}$ & -1.546936 & -1.662932 & 1.635310 & $\mathrm{H}$ & 1.441176 & 1.855415 & -1.907716 \\
\hline $\mathrm{H}$ & -3.480600 & -1.030160 & -2.613627 & $\mathrm{H}$ & 1.933499 & 0.908495 & -0.509095 \\
\hline $\mathrm{H}$ & -3.885366 & -2.706348 & -2.298199 & $\mathrm{H}$ & 3.048075 & 3.625753 & -1.341373 \\
\hline $\mathrm{H}$ & -5.105056 & -2.100577 & -0.271129 & $\mathrm{H}$ & 3.709213 & 3.129207 & 1.087770 \\
\hline $\mathrm{H}$ & -2.636540 & -2.696582 & -0.190690 & $\mathrm{H}$ & -1.430562 & 4.212172 & -0.399736 \\
\hline $\mathrm{H}$ & -1.604533 & -2.209055 & -1.528343 & $\mathrm{H}$ & -0.815188 & 3.651409 & 1.185734 \\
\hline
\end{tabular}




\begin{tabular}{|c|c|c|c|c|c|c|c|}
\hline $\mathrm{H}$ & -0.354887 & 5.261751 & 0.569397 & 0 & 3.133379 & -4.096473 & 0.225540 \\
\hline & nergy $=-193$ & 38.5897684 & 2 a.u. & $\mathrm{C}$ & 4.763258 & 0.350371 & 0.009853 \\
\hline & & & & $\mathrm{S}$ & 4.171849 & 1.395341 & 1.380492 \\
\hline & $S, 10 R, 13 R, 24$ & $R)-\mathbf{1}, \operatorname{Conf} C$ & & $\mathrm{C}$ & 3.186333 & 2.660018 & 0.532607 \\
\hline & & & & $\mathrm{C}$ & 1.832603 & 2.180535 & 0.012702 \\
\hline $\mathrm{C}$ & -3.668555 & -0.613314 & -2.159637 & $\mathrm{C}$ & 1.019033 & 3.365995 & -0.487371 \\
\hline $\mathrm{C}$ & -4.879682 & -0.749013 & -1.254093 & 0 & 1.969058 & 1.243786 & -1.028863 \\
\hline $\mathrm{C}$ & -4.688185 & -0.075960 & 0.123893 & 0 & 0.635328 & 4.166343 & 0.491442 \\
\hline $\mathrm{C}$ & -3.358572 & -0.612552 & 0.739005 & 0 & 0.776581 & 3.539286 & -1.658711 \\
\hline $\mathrm{C}$ & -2.080424 & -0.554205 & -0.156285 & $\mathrm{C}$ & -0.124144 & 5.328649 & 0.110893 \\
\hline $\mathrm{C}$ & -2.415680 & -1.191812 & -1.515068 & $\mathrm{H}$ & -1.451398 & -2.407336 & 0.714558 \\
\hline $\mathrm{C}$ & -3.083244 & -0.068258 & 2.143387 & $\mathrm{H}$ & -3.515412 & 0.440354 & -2.417738 \\
\hline $\mathrm{C}$ & -1.945625 & -0.829844 & 2.824024 & $\mathrm{H}$ & -3.875492 & -1.134168 & -3.097743 \\
\hline $\mathrm{C}$ & -0.720258 & -0.873523 & 1.953687 & $\mathrm{H}$ & -5.053162 & -1.815786 & -1.071845 \\
\hline $\mathrm{C}$ & -0.992375 & -1.422054 & 0.567693 & $\mathrm{H}$ & -2.556426 & -2.270959 & -1.375267 \\
\hline $\mathrm{C}$ & -1.530523 & 0.861396 & -0.380466 & $\mathrm{H}$ & -1.580905 & -1.068967 & -2.209580 \\
\hline $\mathrm{H}$ & -3.546350 & -1.689673 & 0.864791 & $\mathrm{H}$ & -3.978179 & -0.150235 & 2.761980 \\
\hline C & -4.761733 & 1.452138 & 0.000029 & $\mathrm{H}$ & -2.831013 & 0.995019 & 2.101129 \\
\hline $\mathrm{C}$ & -5.860613 & -0.519315 & 1.012847 & $\mathrm{H}$ & -1.708641 & -0.382747 & 3.791757 \\
\hline 0 & -6.063295 & -0.306477 & -1.918904 & $\mathrm{H}$ & -2.276826 & -1.858435 & 3.012370 \\
\hline $\mathrm{C}$ & 0.271893 & -1.652793 & -0.258919 & $\mathrm{H}$ & -0.572224 & 0.808410 & -0.903033 \\
\hline $\mathrm{C}$ & 1.120569 & -2.794148 & 0.234459 & $\mathrm{H}$ & -2.191731 & 1.469773 & -0.994500 \\
\hline $\mathrm{C}$ & 2.614520 & -2.843513 & -0.158188 & $\mathrm{H}$ & -1.364665 & 1.395043 & 0.557521 \\
\hline 0 & 0.686217 & -3.705951 & 0.903852 & $\mathrm{H}$ & -4.466542 & 1.934185 & 0.933637 \\
\hline $\mathrm{C}$ & 2.862545 & -2.562350 & -1.649956 & $\mathrm{H}$ & -5.790408 & 1.758648 & -0.204822 \\
\hline $\mathrm{C}$ & 3.645427 & -1.264201 & -1.690808 & $\mathrm{H}$ & -4.133119 & 1.857322 & -0.792805 \\
\hline $\mathrm{C}$ & 3.371657 & -1.728338 & 0.539458 & $\mathrm{H}$ & -6.802263 & -0.363805 & 0.483651 \\
\hline $\mathrm{C}$ & 3.932333 & -0.854036 & -0.290981 & $\mathrm{H}$ & -5.907509 & 0.052299 & 1.941293 \\
\hline $\mathrm{C}$ & 0.458874 & -0.441697 & 2.387457 & $\mathrm{H}$ & -5.783584 & -1.580150 & 1.268669 \\
\hline 0 & 3.990517 & -0.678224 & -2.690795 & $\mathrm{H}$ & -5.918125 & 0.592727 & -2.227552 \\
\hline
\end{tabular}




\begin{tabular}{|c|c|c|c|c|c|c|c|}
\hline $\mathrm{H}$ & -0.006556 & -1.942734 & -1.277070 & $\mathrm{C}$ & -2.543309 & 0.974288 & 0.180115 \\
\hline $\mathrm{H}$ & 0.882676 & -0.753462 & -0.368769 & $\mathrm{C}$ & -2.703197 & -1.176979 & -1.142072 \\
\hline $\mathrm{H}$ & 3.479240 & -3.364195 & -2.057500 & $\mathrm{H}$ & -5.095008 & 0.749893 & 0.495085 \\
\hline $\mathrm{H}$ & 1.966772 & -2.484988 & -2.265976 & $\mathrm{C}$ & -5.884208 & -1.972193 & -1.521276 \\
\hline $\mathrm{H}$ & 3.429892 & -1.704762 & 1.622397 & $\mathrm{C}$ & -7.307293 & -0.329825 & -0.338310 \\
\hline $\mathrm{H}$ & 1.355183 & -0.452552 & 1.777321 & 0 & -6.757298 & -2.829490 & 1.118734 \\
\hline $\mathrm{H}$ & 0.570835 & -0.053067 & 3.394501 & $\mathrm{C}$ & -1.127618 & 0.754829 & 0.709496 \\
\hline $\mathrm{H}$ & 2.442291 & -4.521625 & 0.757217 & $\mathrm{C}$ & -0.513126 & 1.993223 & 1.308120 \\
\hline $\mathrm{H}$ & 4.848649 & 0.963420 & -0.887786 & $\mathrm{C}$ & 1.025913 & 2.083786 & 1.440656 \\
\hline $\mathrm{H}$ & 5.772609 & 0.042262 & 0.288225 & 0 & -1.164541 & 2.918956 & 1.735732 \\
\hline $\mathrm{H}$ & 3.033336 & 3.438872 & 1.281385 & $\mathrm{C}$ & 1.645629 & 0.805320 & 2.033946 \\
\hline $\mathrm{H}$ & 3.771733 & 3.085422 & -0.285557 & $\mathrm{C}$ & 2.371277 & 0.148802 & 0.882156 \\
\hline $\mathrm{H}$ & 1.277211 & 1.710852 & 0.830266 & $\mathrm{C}$ & 1.662739 & 2.170536 & 0.067193 \\
\hline $\mathrm{H}$ & 1.814203 & 1.709318 & -1.861982 & $\mathrm{C}$ & 2.398143 & 1.106448 & -0.251759 \\
\hline $\mathrm{H}$ & -0.346639 & 5.846892 & 1.038308 & $\mathrm{C}$ & -1.543827 & 2.196203 & -1.791309 \\
\hline $\mathrm{H}$ & 0.466978 & 5.961011 & -0.549936 & 0 & 2.845226 & -0.968421 & 0.887574 \\
\hline $\mathrm{H}$ & -1.042883 & 5.024964 & -0.389216 & 0 & 1.343756 & 3.221468 & 2.201135 \\
\hline \multirow{2}{*}{\multicolumn{4}{|c|}{$\omega B 97 X$ Energy = -1938.58911835 a.u. }} & $\mathrm{C}$ & 3.158093 & 0.839963 & -1.503341 \\
\hline & & & & $S$ & 4.956249 & 1.031992 & -1.196304 \\
\hline \multirow{2}{*}{\multicolumn{4}{|c|}{$(3 S, 5 R, 9 S, 10 R, 13 R, 24 R)-\mathbf{1}$, Conf D }} & $\mathrm{C}$ & 5.653533 & -0.350269 & -2.149453 \\
\hline & & & & $\mathrm{C}$ & 5.691491 & -1.682113 & -1.398689 \\
\hline $\mathrm{C}$ & -4.398285 & -2.283609 & 1.274786 & $\mathrm{C}$ & 6.598107 & -1.548388 & -0.176076 \\
\hline $\mathrm{C}$ & -5.803048 & -1.773583 & 1.005793 & 0 & 4.423907 & -2.211084 & -1.121134 \\
\hline $\mathrm{C}$ & -5.927160 & -1.005577 & -0.329730 & 0 & 6.015831 & -1.927462 & 0.951949 \\
\hline $\mathrm{C}$ & -4.826196 & 0.099110 & -0.350745 & 0 & 7.731020 & -1.134809 & -0.248946 \\
\hline $\mathrm{C}$ & -3.362531 & -0.345004 & -0.034270 & $\mathrm{C}$ & 6.802211 & -1.805281 & 2.147548 \\
\hline $\mathrm{C}$ & -3.377651 & -1.152417 & 1.274143 & $\mathrm{H}$ & -3.077992 & 1.530655 & 0.959130 \\
\hline $\mathrm{C}$ & -4.887456 & 0.984774 & -1.598089 & $\mathrm{H}$ & -4.130609 & -3.042572 & 0.531565 \\
\hline $\mathrm{C}$ & -3.995938 & 2.219131 & -1.455204 & $\mathrm{H}$ & -4.390950 & -2.785979 & 2.245157 \\
\hline $\mathrm{C}$ & -2.595768 & 1.832891 & -1.066483 & $\mathrm{H}$ & -6.073410 & -1.075493 & 1.806236 \\
\hline
\end{tabular}




\begin{tabular}{|c|c|c|c|c|c|c|c|}
\hline $\mathrm{H}$ & -3.601620 & -0.474270 & 2.107090 & $\mathrm{H}$ & 7.704534 & -2.410284 & 2.068465 \\
\hline $\mathrm{H}$ & -2.390421 & -1.578722 & 1.468912 & $\mathrm{H}$ & 7.071720 & -0.762651 & 2.312713 \\
\hline $\mathrm{H}$ & -5.912407 & 1.313014 & -1.776592 & $\mathrm{H}$ & 6.168977 & -2.167028 & 2.952006 \\
\hline $\mathrm{H}$ & -4.582149 & 0.422214 & -2.484711 & \multicolumn{4}{|c|}{$\omega \mathrm{B} 97 \mathrm{X}$ Energy = -1938.58899066 a.u. } \\
\hline $\mathrm{H}$ & -3.987657 & 2.796352 & -2.382203 & & & & \\
\hline $\mathrm{H}$ & -4.412364 & 2.864770 & -0.672357 & \multicolumn{4}{|c|}{$(3 S, 5 R, 9 S, 10 R, 13 R, 24 R)-\mathbf{1}$, Conf $\mathrm{E}$} \\
\hline $\mathrm{H}$ & -3.152736 & -2.163281 & -1.237436 & & & & \\
\hline $\mathrm{H}$ & -2.758951 & -0.690458 & -2.116273 & $\mathrm{C}$ & -3.987664 & -0.030834 & 2.194910 \\
\hline $\mathrm{H}$ & -1.645136 & -1.333925 & -0.917088 & $\mathrm{C}$ & -5.203103 & 0.040351 & 1.287782 \\
\hline $\mathrm{H}$ & -5.790837 & -1.429475 & -2.463435 & $\mathrm{C}$ & -4.952321 & -0.561974 & -0.113586 \\
\hline $\mathrm{H}$ & -6.817189 & -2.538845 & -1.572967 & $\mathrm{C}$ & -3.674040 & 0.112402 & -0.700121 \\
\hline $\mathrm{H}$ & -5.065915 & -2.690886 & -1.473966 & $\mathrm{C}$ & -2.395535 & 0.122979 & 0.197605 \\
\hline $\mathrm{H}$ & -8.074873 & -1.061297 & -0.080308 & $\mathrm{C}$ & -2.783584 & 0.671163 & 1.581008 \\
\hline $\mathrm{H}$ & -7.556106 & 0.076429 & -1.320095 & $\mathrm{C}$ & -3.352672 & -0.342815 & -2.126473 \\
\hline $\mathrm{H}$ & -7.353768 & 0.484956 & 0.390205 & $\mathrm{C}$ & -2.280901 & 0.539090 & -2.768163 \\
\hline $\mathrm{H}$ & -6.497657 & -3.536195 & 0.520283 & $\mathrm{C}$ & -1.066601 & 0.653614 & -1.889112 \\
\hline $\mathrm{H}$ & -1.146159 & 0.030124 & 1.528969 & $\mathrm{C}$ & -1.391143 & 1.114925 & -0.483407 \\
\hline $\mathrm{H}$ & -0.457038 & 0.335857 & -0.043384 & $\mathrm{C}$ & -1.726569 & -1.248407 & 0.360468 \\
\hline $\mathrm{H}$ & 2.386455 & 1.108455 & 2.776101 & $\mathrm{H}$ & -3.953487 & 1.174024 & -0.778953 \\
\hline $\mathrm{H}$ & 0.952114 & 0.116423 & 2.514602 & C & -4.888898 & -2.093756 & -0.045879 \\
\hline $\mathrm{H}$ & 1.510867 & 3.047988 & -0.552132 & $\mathrm{C}$ & -6.157954 & -0.190671 & -0.990761 \\
\hline $\mathrm{H}$ & -0.527620 & 1.908444 & -1.546232 & 0 & -6.348403 & -0.522357 & 1.928088 \\
\hline $\mathrm{H}$ & -1.668671 & 2.801935 & -2.682616 & $\mathrm{C}$ & -0.151969 & 1.438930 & 0.351081 \\
\hline $\mathrm{H}$ & 0.511730 & 3.704864 & 2.326184 & $\mathrm{C}$ & 0.537009 & 2.706579 & -0.083283 \\
\hline $\mathrm{H}$ & 2.850845 & 1.522976 & -2.293286 & $\mathrm{C}$ & 2.030810 & 2.915125 & 0.254735 \\
\hline $\mathrm{H}$ & 2.981838 & -0.184184 & -1.832141 & 0 & -0.031146 & 3.602194 & -0.668543 \\
\hline $\mathrm{H}$ & 5.093197 & -0.468976 & -3.076393 & $\mathrm{C}$ & 2.385503 & 2.562060 & 1.708962 \\
\hline $\mathrm{H}$ & 6.670580 & -0.054104 & -2.405253 & $\mathrm{C}$ & 3.317420 & 1.371308 & 1.616882 \\
\hline $\mathrm{H}$ & 6.191715 & -2.402605 & -2.053628 & $\mathrm{C}$ & 2.877810 & 1.953808 & -0.557957 \\
\hline $\mathrm{H}$ & 4.006333 & -1.733672 & -0.382577 & $\mathrm{C}$ & 3.576527 & 1.095213 & 0.179658 \\
\hline
\end{tabular}




\begin{tabular}{|c|c|c|c|c|c|c|c|}
\hline $\mathrm{C}$ & 0.149450 & 0.358018 & -2.337393 & $\mathrm{H}$ & -6.177339 & 0.882325 & -1.203494 \\
\hline 0 & 3.779964 & 0.756863 & 2.551246 & $\mathrm{H}$ & -6.135092 & -1.422708 & 2.190401 \\
\hline 0 & 2.376117 & 4.245005 & -0.053166 & $\mathrm{H}$ & -0.444336 & 1.629553 & 1.388464 \\
\hline $\mathrm{C}$ & 4.523243 & 0.020416 & -0.242632 & $\mathrm{H}$ & 0.562563 & 0.613936 & 0.390291 \\
\hline $\mathrm{S}$ & 4.079207 & -0.776129 & -1.827517 & $\mathrm{H}$ & 2.920359 & 3.403183 & 2.151258 \\
\hline $\mathrm{C}$ & 3.953317 & -2.531926 & -1.378309 & $\mathrm{H}$ & 1.538094 & 2.329894 & 2.354099 \\
\hline $\mathrm{C}$ & 2.609986 & -2.967095 & -0.779191 & $\mathrm{H}$ & 2.884622 & 2.020077 & -1.640615 \\
\hline $\mathrm{C}$ & 2.413099 & -2.377045 & 0.609802 & $\mathrm{H}$ & 1.040178 & 0.437364 & -1.723026 \\
\hline 0 & 1.518338 & -2.665512 & -1.606306 & $\mathrm{H}$ & 0.299660 & 0.035108 & -3.362702 \\
\hline 0 & 3.124735 & -3.021598 & 1.522452 & $\mathrm{H}$ & 1.616684 & 4.616333 & -0.529244 \\
\hline 0 & 1.710629 & -1.420338 & 0.836607 & $\mathrm{H}$ & 4.581280 & -0.736522 & 0.541003 \\
\hline $\mathrm{C}$ & 3.033346 & -2.536283 & 2.876706 & $\mathrm{H}$ & 5.526714 & 0.436130 & -0.346423 \\
\hline $\mathrm{H}$ & -1.939519 & 2.057856 & -0.595258 & $\mathrm{H}$ & 4.086710 & -3.067798 & -2.318119 \\
\hline $\mathrm{H}$ & -3.751979 & -1.077815 & 2.415529 & $\mathrm{H}$ & 4.777399 & -2.807322 & -0.719037 \\
\hline $\mathrm{H}$ & -4.238908 & 0.436801 & 3.150073 & $\mathrm{H}$ & 2.639935 & -4.053388 & -0.674831 \\
\hline $\mathrm{H}$ & -5.462323 & 1.095562 & 1.144334 & $\mathrm{H}$ & 1.332462 & -1.719379 & -1.538218 \\
\hline $\mathrm{H}$ & -3.007964 & 1.741263 & 1.488198 & $\mathrm{H}$ & 3.375643 & -1.503067 & 2.927555 \\
\hline $\mathrm{H}$ & -1.942369 & 0.583748 & 2.273332 & $\mathrm{H}$ & 2.003358 & -2.606583 & 3.224561 \\
\hline $\mathrm{H}$ & -4.250331 & -0.305603 & -2.745418 & $\mathrm{H}$ & 3.676297 & -3.187334 & 3.461182 \\
\hline $\mathrm{H}$ & -3.014134 & -1.382431 & -2.132382 & \multicolumn{4}{|c|}{$\omega$ B97X Energy = -1938.58866315 a.u. } \\
\hline $\mathrm{H}$ & -2.003683 & 0.153668 & -3.751556 & & & & \\
\hline $\mathrm{H}$ & -2.697158 & 1.542984 & -2.916866 & \multicolumn{4}{|c|}{$(3 S, 5 R, 9 S, 10 R, 13 R, 24 R)-\mathbf{1}$, Conf $\mathrm{F}$} \\
\hline $\mathrm{H}$ & -1.558533 & -1.746649 & -0.595621 & & & & \\
\hline $\mathrm{H}$ & -0.750325 & -1.140518 & 0.838242 & $\mathrm{C}$ & -3.690151 & 1.728691 & -2.161631 \\
\hline $\mathrm{H}$ & -2.315522 & -1.918987 & 0.983733 & $\mathrm{C}$ & -5.048166 & 1.874494 & -1.498597 \\
\hline $\mathrm{H}$ & -5.884539 & -2.499814 & 0.149760 & $\mathrm{C}$ & -4.971642 & 1.912937 & 0.044940 \\
\hline $\mathrm{H}$ & -4.220931 & -2.468681 & 0.729315 & $\mathrm{C}$ & -4.158730 & 0.666820 & 0.513064 \\
\hline $\mathrm{H}$ & -4.557082 & -2.513905 & -0.996960 & $\mathrm{C}$ & -2.770202 & 0.425622 & -0.161455 \\
\hline $\mathrm{H}$ & -7.082785 & -0.452076 & -0.473759 & $\mathrm{C}$ & -2.960224 & 0.478811 & -1.686597 \\
\hline $\mathrm{H}$ & -6.150193 & -0.726262 & -1.941589 & $\mathrm{C}$ & -4.073747 & 0.541591 & 2.037283 \\
\hline
\end{tabular}




\begin{tabular}{|c|c|c|c|c|c|c|c|}
\hline $\mathrm{C}$ & -3.527656 & -0.823786 & 2.458813 & $\mathrm{H}$ & -3.835503 & 1.680971 & -3.243640 \\
\hline $\mathrm{C}$ & -2.227695 & -1.117417 & 1.763803 & $\mathrm{H}$ & -5.658062 & 1.005363 & -1.770911 \\
\hline $\mathrm{C}$ & -2.330266 & -1.020424 & 0.255839 & $\mathrm{H}$ & -3.528907 & -0.404012 & -2.004760 \\
\hline $\mathrm{C}$ & -1.682966 & 1.426013 & 0.253368 & $\mathrm{H}$ & -1.992191 & 0.429843 & -2.191547 \\
\hline $\mathrm{H}$ & -4.769306 & -0.183950 & 0.174192 & $\mathrm{H}$ & -5.060964 & 0.676351 & 2.481627 \\
\hline $\mathrm{C}$ & -4.412113 & 3.257144 & 0.530773 & $\mathrm{H}$ & -3.435491 & 1.326599 & 2.452500 \\
\hline $\mathrm{C}$ & -6.409708 & 1.796720 & 0.573167 & $\mathrm{H}$ & -3.398999 & -0.869043 & 3.542269 \\
\hline 0 & -5.758263 & 2.989296 & -2.038192 & $\mathrm{H}$ & -4.257635 & -1.594494 & 2.183258 \\
\hline $\mathrm{C}$ & -1.092743 & -1.535588 & -0.475899 & $\mathrm{H}$ & -0.710237 & 1.106775 & -0.130582 \\
\hline $\mathrm{C}$ & -0.911809 & -3.028417 & -0.374124 & $\mathrm{H}$ & -1.865537 & 2.420253 & -0.149010 \\
\hline $\mathrm{C}$ & 0.494951 & -3.635252 & -0.602112 & $\mathrm{H}$ & -1.588696 & 1.516627 & 1.335727 \\
\hline 0 & -1.822689 & -3.796827 & -0.166049 & $\mathrm{H}$ & -4.199716 & 3.227791 & 1.600878 \\
\hline $\mathrm{C}$ & 1.188942 & -3.046564 & -1.843449 & $\mathrm{H}$ & -5.152538 & 4.045791 & 0.375454 \\
\hline $\mathrm{C}$ & 2.244791 & -2.116280 & -1.294406 & $\mathrm{H}$ & -3.496968 & 3.559259 & 0.021554 \\
\hline $\mathrm{C}$ & 1.413248 & -3.223068 & 0.530363 & $\mathrm{H}$ & -7.047618 & 2.517533 & 0.058847 \\
\hline $\mathrm{C}$ & 2.363473 & -2.363387 & 0.163782 & $\mathrm{H}$ & -6.470742 & 2.007451 & 1.642260 \\
\hline $\mathrm{C}$ & -1.116230 & -1.381509 & 2.441118 & $\mathrm{H}$ & -6.816282 & 0.796137 & 0.399261 \\
\hline 0 & 2.890408 & -1.319577 & -1.945295 & $\mathrm{H}$ & -5.205269 & 3.771040 & -1.948727 \\
\hline 0 & 0.376786 & -5.031707 & -0.673676 & $\mathrm{H}$ & -1.191223 & -1.343423 & -1.548701 \\
\hline $\mathrm{C}$ & 3.372676 & -1.677088 & 1.016824 & $\mathrm{H}$ & -0.178720 & -1.026613 & -0.163595 \\
\hline S & 2.803877 & 0.039423 & 1.336851 & $\mathrm{H}$ & 1.690024 & -3.865941 & -2.362473 \\
\hline $\mathrm{C}$ & 4.333682 & 1.001481 & 1.170760 & $\mathrm{H}$ & 0.542036 & -2.541134 & -2.559359 \\
\hline $\mathrm{C}$ & 4.699243 & 1.348074 & -0.276501 & $\mathrm{H}$ & 1.262677 & -3.618952 & 1.528948 \\
\hline $\mathrm{C}$ & 5.899358 & 2.287849 & -0.261037 & $\mathrm{H}$ & -0.160702 & -1.562898 & 1.962185 \\
\hline 0 & 5.041541 & 0.224335 & -1.041642 & $\mathrm{H}$ & -1.123303 & -1.416205 & 3.525427 \\
\hline 0 & 5.556278 & 3.474239 & 0.241308 & $\mathrm{H}$ & -0.539384 & -5.239361 & -0.430972 \\
\hline 0 & 7.011337 & 2.006729 & -0.624846 & $\mathrm{H}$ & 4.334680 & -1.624411 & 0.509287 \\
\hline $\mathrm{C}$ & 6.607894 & 4.444689 & 0.358199 & $\mathrm{H}$ & 3.497499 & -2.197596 & 1.964481 \\
\hline $\mathrm{H}$ & -3.155877 & -1.678738 & -0.039533 & $\mathrm{H}$ & 4.159101 & 1.919318 & 1.732684 \\
\hline $\mathrm{H}$ & -3.087865 & 2.623452 & -1.969788 & $\mathrm{H}$ & 5.152942 & 0.461785 & 1.649549 \\
\hline
\end{tabular}




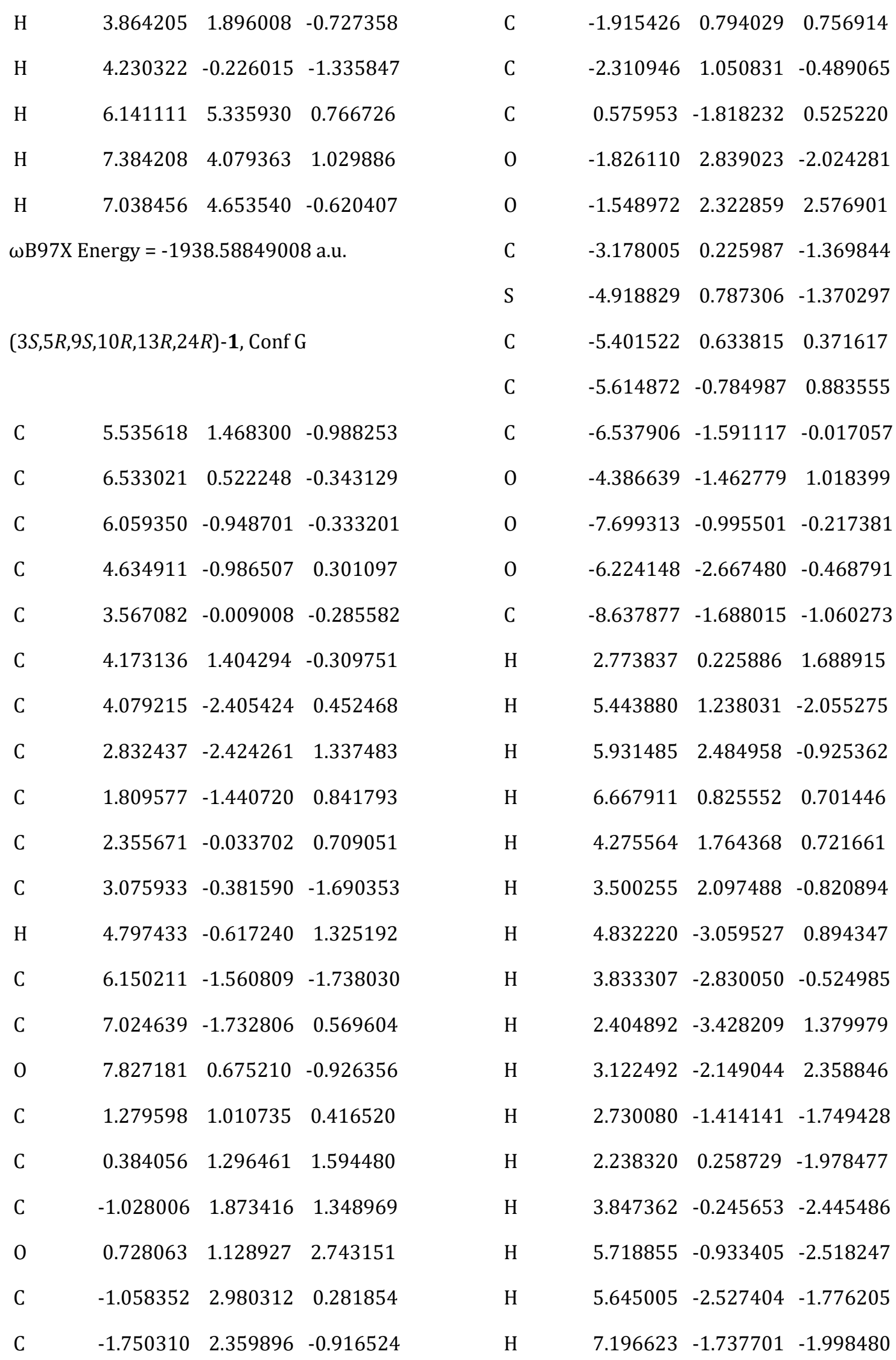




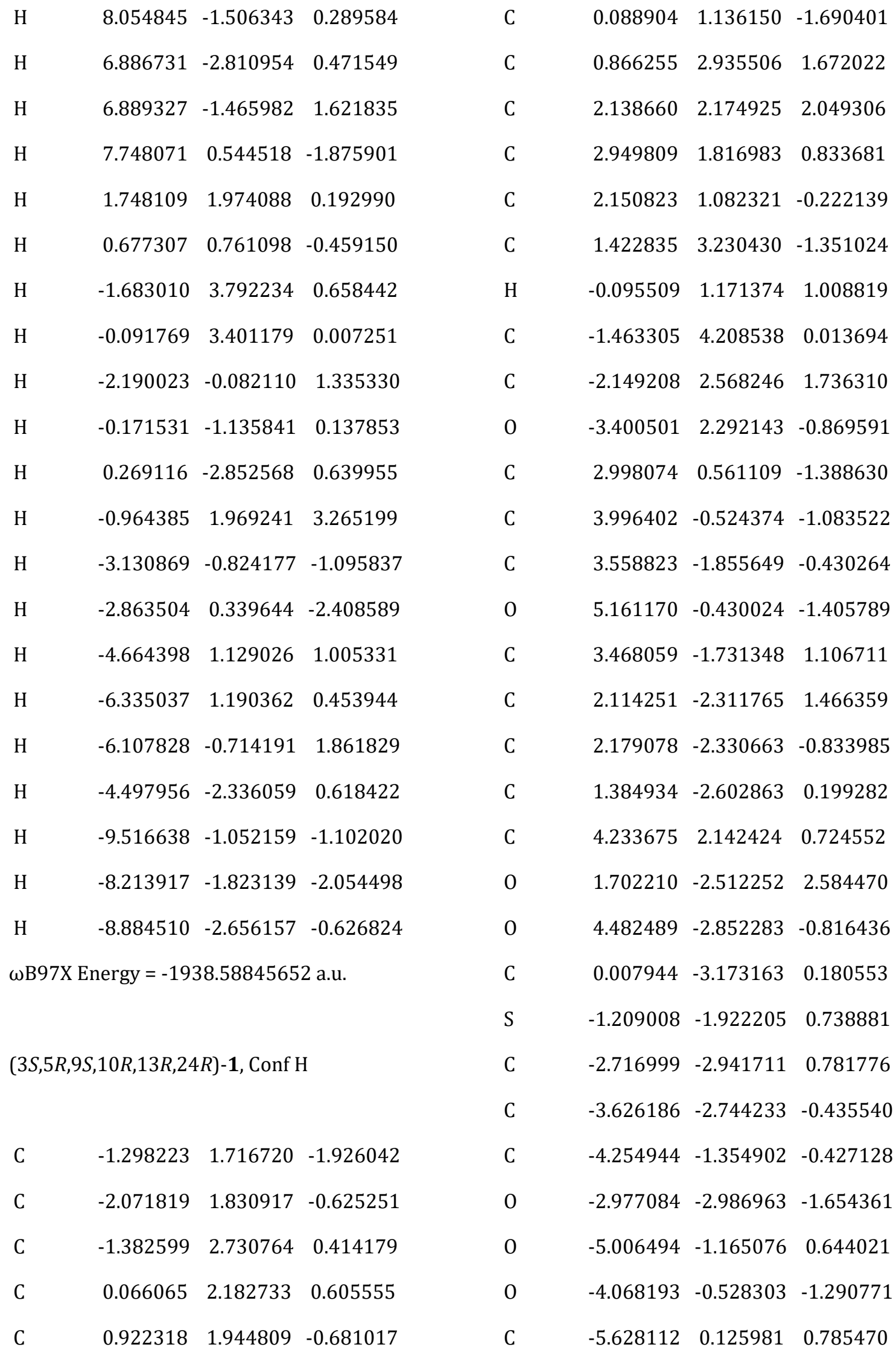




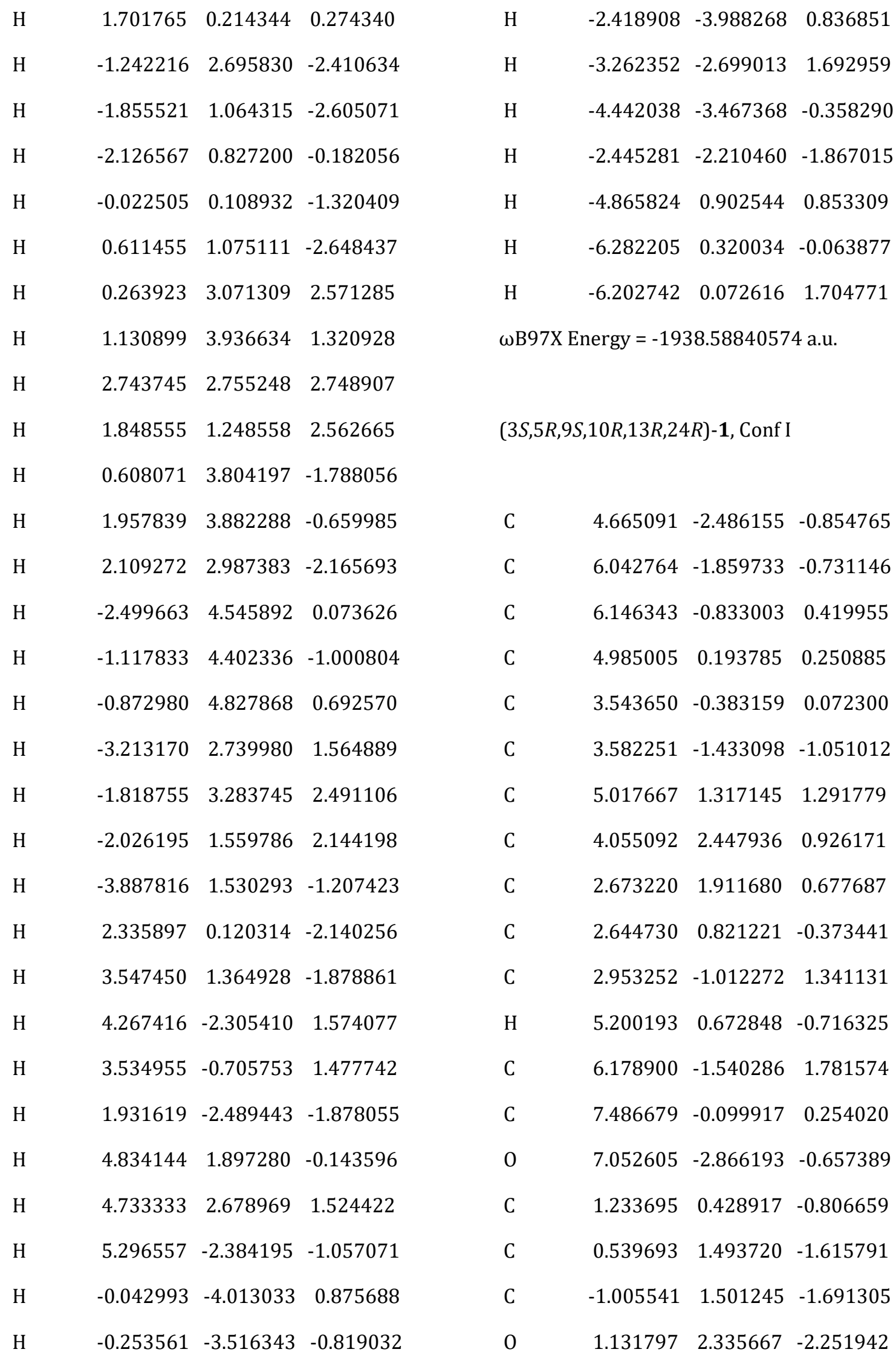




\begin{tabular}{|c|c|c|c|c|c|c|c|}
\hline $\mathrm{C}$ & -1.609212 & 0.105127 & -1.917388 & $\mathrm{H}$ & 5.389854 & -2.281140 & 1.909373 \\
\hline $\mathrm{C}$ & -2.334311 & -0.228424 & -0.633710 & $\mathrm{H}$ & 6.090559 & -0.820458 & 2.597126 \\
\hline $\mathrm{C}$ & -1.581322 & 1.914319 & -0.350727 & $\mathrm{H}$ & 8.288454 & -0.829131 & 0.125987 \\
\hline $\mathrm{C}$ & -2.311931 & 0.969797 & 0.241843 & $\mathrm{H}$ & 7.729885 & 0.506855 & 1.128026 \\
\hline $\mathrm{C}$ & 1.621545 & 2.342532 & 1.364979 & $\mathrm{H}$ & 7.475293 & 0.554034 & -0.622922 \\
\hline 0 & -2.851607 & -1.297978 & -0.378872 & $\mathrm{H}$ & 6.840848 & -3.452272 & 0.075099 \\
\hline 0 & -1.400766 & 2.407100 & -2.691129 & $\mathrm{H}$ & 1.278963 & -0.437474 & -1.473707 \\
\hline C & -2.986412 & 1.026000 & 1.572357 & $\mathrm{H}$ & 0.602433 & 0.127694 & 0.031579 \\
\hline$S$ & -4.688831 & 1.687152 & 1.544781 & $\mathrm{H}$ & -2.342875 & 0.174719 & -2.722120 \\
\hline $\mathrm{C}$ & -5.585207 & 0.513903 & 0.493915 & $\mathrm{H}$ & -0.901714 & -0.680598 & -2.179939 \\
\hline $\mathrm{C}$ & -5.950147 & -0.812622 & 1.169055 & $\mathrm{H}$ & -1.397772 & 2.910042 & 0.039096 \\
\hline $\mathrm{C}$ & -6.765958 & -1.635680 & 0.172411 & $\mathrm{H}$ & 0.622083 & 1.945490 & 1.228326 \\
\hline 0 & -4.846935 & -1.539824 & 1.632172 & $\mathrm{H}$ & 1.730734 & 3.118527 & 2.115204 \\
\hline 0 & -8.001951 & -1.158231 & 0.049545 & $\mathrm{H}$ & -0.603146 & 2.899949 & -2.940580 \\
\hline 0 & -6.335756 & -2.574208 & -0.449352 & $\mathrm{H}$ & -2.450353 & 1.710649 & 2.229717 \\
\hline $\mathrm{C}$ & -8.846602 & -1.808633 & -0.913404 & $\mathrm{H}$ & -2.993065 & 0.042662 & 2.038455 \\
\hline $\mathrm{H}$ & 3.130157 & 1.240426 & -1.262732 & $\mathrm{H}$ & -6.502345 & 1.033491 & 0.213752 \\
\hline $\mathrm{H}$ & 4.453845 & -3.094431 & 0.031572 & $\mathrm{H}$ & -5.025011 & 0.337091 & -0.426787 \\
\hline $\mathrm{H}$ & 4.670722 & -3.172675 & -1.704936 & $\mathrm{H}$ & -6.583301 & -0.606839 & 2.034190 \\
\hline $\mathrm{H}$ & 6.259905 & -1.324555 & -1.662667 & $\mathrm{H}$ & -4.193967 & -1.617876 & 0.916385 \\
\hline $\mathrm{H}$ & 3.753709 & -0.923765 & -2.007618 & $\mathrm{H}$ & -9.799682 & -1.290645 & -0.865124 \\
\hline $\mathrm{H}$ & 2.616592 & -1.939032 & -1.131560 & $\mathrm{H}$ & -8.415051 & -1.722131 & -1.910093 \\
\hline $\mathrm{H}$ & 6.025452 & 1.727338 & 1.371473 & $\mathrm{H}$ & -8.970677 & -2.859960 & -0.656525 \\
\hline $\mathrm{H}$ & 4.758632 & 0.931848 & 2.282014 & \multicolumn{4}{|c|}{$\omega \mathrm{B} 97 \mathrm{X}$ Energy = -1938.58823957 a.u. } \\
\hline $\mathrm{H}$ & 4.034908 & 3.205140 & 1.712651 & & & & \\
\hline $\mathrm{H}$ & 4.415918 & 2.934405 & 0.011923 & \multicolumn{4}{|c|}{$(3 S, 5 R, 9 S, 10 R, 13 R, 24 R)-\mathbf{1}$, Conf J } \\
\hline $\mathrm{H}$ & 3.014206 & -0.346271 & 2.202213 & & & & \\
\hline $\mathrm{H}$ & 1.897369 & -1.248766 & 1.187045 & $\mathrm{C}$ & -5.125791 & 1.686242 & 1.433433 \\
\hline $\mathrm{H}$ & 3.446628 & -1.945203 & 1.605690 & $\mathrm{C}$ & -6.304918 & 1.263865 & 0.575081 \\
\hline $\mathrm{H}$ & 7.136185 & -2.051144 & 1.911694 & $\mathrm{C}$ & -6.234861 & -0.214663 & 0.129649 \\
\hline
\end{tabular}




\begin{tabular}{|c|c|c|c|c|c|c|c|}
\hline $\mathrm{C}$ & -4.841041 & -0.449052 & -0.529626 & 0 & 7.214453 & 1.253537 & 0.381904 \\
\hline $\mathrm{C}$ & -3.582234 & 0.006596 & 0.275427 & $\mathrm{C}$ & 5.231228 & 2.437183 & -0.977789 \\
\hline $\mathrm{C}$ & -3.798450 & 1.462956 & 0.720115 & $\mathrm{H}$ & -2.672394 & 0.565317 & -1.581365 \\
\hline C & -4.670189 & -1.864145 & -1.089553 & $\mathrm{H}$ & -5.146978 & 1.143530 & 2.384712 \\
\hline $\mathrm{C}$ & -3.432104 & -1.965405 & -1.981534 & $\mathrm{H}$ & -5.238221 & 2.744961 & 1.679653 \\
\hline $\mathrm{C}$ & -2.211227 & -1.461999 & -1.263450 & $\mathrm{H}$ & -6.304270 & 1.876107 & -0.334159 \\
\hline $\mathrm{C}$ & -2.377955 & -0.055148 & -0.726450 & $\mathrm{H}$ & -3.758213 & 2.113865 & -0.162293 \\
\hline $\mathrm{C}$ & -3.264711 & -0.859812 & 1.501236 & $\mathrm{H}$ & -2.991565 & 1.781085 & 1.385130 \\
\hline $\mathrm{H}$ & -4.852092 & 0.220577 & -1.403148 & $\mathrm{H}$ & -5.547300 & -2.145757 & -1.674032 \\
\hline $\mathrm{C}$ & -6.552172 & -1.153028 & 1.301834 & $\mathrm{H}$ & -4.589474 & -2.593092 & -0.278265 \\
\hline $\mathrm{C}$ & -7.327312 & -0.421761 & -0.931203 & $\mathrm{H}$ & -3.280481 & -2.994431 & -2.314152 \\
\hline 0 & -7.539767 & 1.583468 & 1.216565 & $\mathrm{H}$ & -3.593768 & -1.352969 & -2.876888 \\
\hline $\mathrm{C}$ & -1.081928 & 0.549125 & -0.190364 & $\mathrm{H}$ & -3.191976 & -1.919698 & 1.255447 \\
\hline $\mathrm{C}$ & -0.086814 & 0.887340 & -1.270030 & $\mathrm{H}$ & -2.306803 & -0.560002 & 1.933890 \\
\hline $\mathrm{C}$ & 1.408175 & 1.023348 & -0.902710 & $\mathrm{H}$ & -4.009796 & -0.751339 & 2.286771 \\
\hline 0 & -0.401347 & 1.096575 & -2.420133 & $\mathrm{H}$ & -5.994421 & -0.923434 & 2.209680 \\
\hline $\mathrm{C}$ & 1.646122 & 1.812575 & 0.395488 & $\mathrm{H}$ & -6.341702 & -2.191486 & 1.040283 \\
\hline $\mathrm{C}$ & 2.155938 & 0.793819 & 1.395523 & $\mathrm{H}$ & -7.616149 & -1.096332 & 1.544869 \\
\hline $\mathrm{C}$ & 1.983131 & -0.347623 & -0.590697 & $\mathrm{H}$ & -8.273818 & -0.021712 & -0.563819 \\
\hline $\mathrm{C}$ & 2.400135 & -0.480784 & 0.666945 & $\mathrm{H}$ & -7.482027 & -1.478510 & -1.155611 \\
\hline $\mathrm{C}$ & -1.133336 & -2.221254 & -1.102407 & $\mathrm{H}$ & -7.077705 & 0.092555 & -1.863854 \\
\hline 0 & 2.321190 & 0.976378 & 2.578894 & $\mathrm{H}$ & -7.544459 & 1.170310 & 2.085060 \\
\hline 0 & 2.084257 & 1.603784 & -1.993041 & $\mathrm{H}$ & -1.292743 & 1.505971 & 0.296874 \\
\hline $\mathrm{C}$ & 3.026979 & -1.652733 & 1.333414 & $\mathrm{H}$ & -0.607448 & -0.074208 & 0.570295 \\
\hline S & 4.826236 & -1.447801 & 1.563751 & $\mathrm{H}$ & 2.431582 & 2.547323 & 0.211186 \\
\hline $\mathrm{C}$ & 5.383797 & -1.702691 & -0.151119 & $\mathrm{H}$ & 0.780565 & 2.344586 & 0.788480 \\
\hline $\mathrm{C}$ & 6.668049 & -0.937502 & -0.424912 & $\mathrm{H}$ & 2.032486 & -1.106273 & -1.364957 \\
\hline $\mathrm{C}$ & 6.483599 & 0.568623 & -0.295024 & $\mathrm{H}$ & -0.252377 & -1.892926 & -0.562800 \\
\hline 0 & 7.692270 & -1.358933 & 0.444146 & $\mathrm{H}$ & -1.099542 & -3.226180 & -1.509901 \\
\hline 0 & 5.476612 & 1.019763 & -1.018755 & $\mathrm{H}$ & 1.463081 & 1.588204 & -2.737896 \\
\hline
\end{tabular}




\begin{tabular}{|c|c|c|c|c|c|c|c|}
\hline $\mathrm{H}$ & 2.836492 & -2.575656 & 0.786681 & $\mathrm{C}$ & 2.494097 & -2.461679 & 1.231027 \\
\hline $\mathrm{H}$ & 2.624801 & -1.762606 & 2.341493 & 0 & 0.570520 & -2.481415 & 2.606791 \\
\hline $\mathrm{H}$ & 5.554627 & -2.763827 & -0.330201 & $\mathrm{C}$ & 2.703751 & -3.321282 & -0.031853 \\
\hline $\mathrm{H}$ & 4.610720 & -1.348002 & -0.832983 & $\mathrm{C}$ & 3.570647 & -2.483508 & -0.953898 \\
\hline $\mathrm{H}$ & 6.956028 & -1.127685 & -1.466821 & $\mathrm{C}$ & 3.253532 & -1.185539 & 0.915876 \\
\hline $\mathrm{H}$ & 8.116066 & -0.561707 & 0.789840 & $\mathrm{C}$ & 3.845765 & -1.187775 & -0.276857 \\
\hline $\mathrm{H}$ & 4.326353 & 2.586054 & -1.560528 & $\mathrm{C}$ & 0.355868 & 0.947490 & 1.849537 \\
\hline $\mathrm{H}$ & 5.085546 & 2.758860 & 0.053517 & 0 & 3.983442 & -2.824007 & -2.037681 \\
\hline $\mathrm{H}$ & 6.073600 & 2.972347 & -1.414510 & 0 & 3.055348 & -3.068324 & 2.371557 \\
\hline & nergy $=-19$ & 38.5880956 & 6 a.u. & $\mathrm{C}$ & 4.699755 & -0.127188 & -0.876805 \\
\hline & & & & $S$ & 3.898079 & 0.745092 & -2.268497 \\
\hline & $, 10 R, 13 R, 24$ & $4 R$ )-1, Conf $\mathrm{K}$ & & $\mathrm{C}$ & 2.444011 & 1.500021 & -1.499661 \\
\hline & & & & $\mathrm{C}$ & 2.717508 & 2.715469 & -0.633076 \\
\hline $\mathrm{C}$ & -3.754577 & -1.802469 & -1.798286 & $\mathrm{C}$ & 1.403241 & 3.426871 & -0.305374 \\
\hline $\mathrm{C}$ & -4.971263 & -1.358468 & -1.005820 & 0 & 3.416095 & 2.309451 & 0.524686 \\
\hline $\mathrm{C}$ & -4.769918 & -0.002505 & -0.291619 & 0 & 1.436654 & 3.985980 & 0.900031 \\
\hline $\mathrm{C}$ & -3.453156 & -0.087884 & 0.541169 & 0 & 0.471882 & 3.512952 & -1.066143 \\
\hline $\mathrm{C}$ & -2.171168 & -0.599245 & -0.190677 & $\mathrm{C}$ & 0.254253 & 4.692263 & 1.309965 \\
\hline $\mathrm{C}$ & -2.519851 & -1.910869 & -0.913224 & $\mathrm{H}$ & -1.598287 & -1.589540 & 1.622803 \\
\hline $\mathrm{C}$ & -3.164001 & 1.181907 & 1.346063 & $\mathrm{H}$ & -3.575900 & -1.106161 & -2.625002 \\
\hline $\mathrm{C}$ & -2.059046 & 0.944573 & 2.375459 & $\mathrm{H}$ & -3.969709 & -2.772877 & -2.252207 \\
\hline $\mathrm{C}$ & -0.831344 & 0.365249 & 1.728726 & $\mathrm{H}$ & -5.167012 & -2.108225 & -0.230743 \\
\hline $\mathrm{C}$ & -1.113349 & -0.891095 & 0.930014 & $\mathrm{H}$ & -2.691361 & -2.694469 & -0.164391 \\
\hline $\mathrm{C}$ & -1.582581 & 0.398481 & -1.196893 & $\mathrm{H}$ & -1.679080 & -2.240395 & -1.528629 \\
\hline $\mathrm{H}$ & -3.666516 & -0.872597 & 1.282802 & $\mathrm{H}$ & -4.063169 & 1.513429 & 1.867340 \\
\hline $\mathrm{C}$ & -4.815745 & 1.155977 & -1.297964 & $\mathrm{H}$ & -2.868514 & 1.999354 & 0.682012 \\
\hline $\mathrm{C}$ & -5.951853 & 0.182152 & 0.672847 & $\mathrm{H}$ & -1.811211 & 1.872427 & 2.896048 \\
\hline 0 & -6.143313 & -1.376436 & -1.820871 & $\mathrm{H}$ & -2.427354 & 0.236758 & 3.128034 \\
\hline $\mathrm{C}$ & 0.144202 & -1.596253 & 0.424334 & $\mathrm{H}$ & -2.256383 & 0.585529 & -2.030158 \\
\hline $\mathrm{C}$ & 1.002057 & -2.178266 & 1.517158 & $\mathrm{H}$ & -1.340008 & 1.360030 & -0.742584 \\
\hline
\end{tabular}




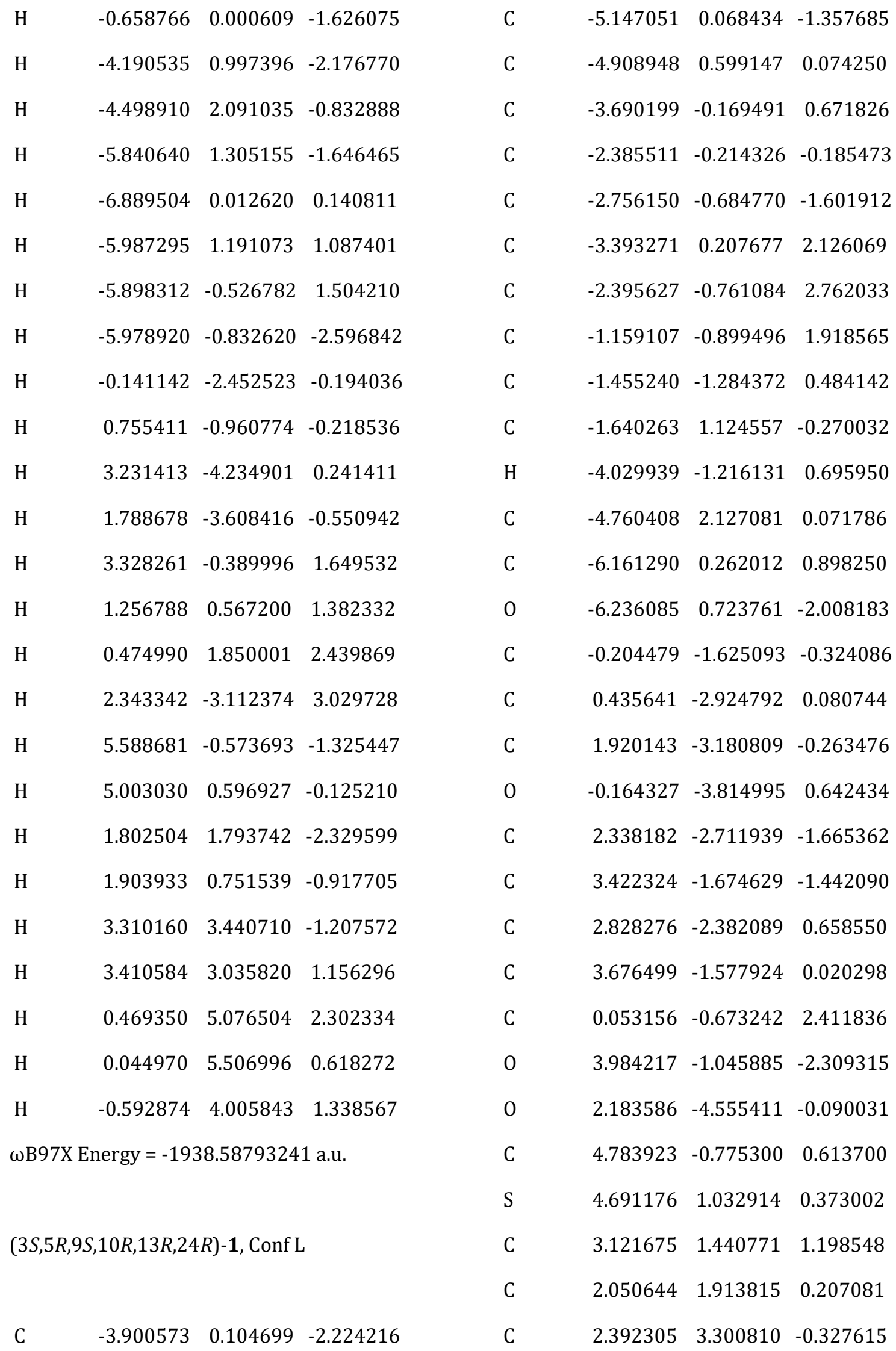




\begin{tabular}{|c|c|c|c|c|c|c|c|}
\hline 0 & 1.851272 & 1.016629 & -0.852801 & $\mathrm{H}$ & 0.190627 & -0.393730 & 3.451000 \\
\hline 0 & 2.277140 & 4.225492 & 0.618847 & $\mathrm{H}$ & 1.428847 & -4.912486 & 0.403903 \\
\hline 0 & 2.743960 & 3.521317 & -1.459294 & $\mathrm{H}$ & 5.728623 & -1.049482 & 0.140019 \\
\hline $\mathrm{C}$ & 2.613486 & 5.571616 & 0.243416 & $\mathrm{H}$ & 4.872076 & -0.983790 & 1.679538 \\
\hline $\mathrm{H}$ & -2.051037 & -2.203852 & 0.532751 & $\mathrm{H}$ & 2.764999 & 0.550365 & 1.714679 \\
\hline $\mathrm{H}$ & -3.601398 & 1.144222 & -2.397925 & $\mathrm{H}$ & 3.301655 & 2.211975 & 1.946681 \\
\hline $\mathrm{H}$ & -4.145833 & -0.313195 & -3.203666 & $\mathrm{H}$ & 1.100829 & 1.987680 & 0.742952 \\
\hline $\mathrm{H}$ & -5.471801 & -0.974377 & -1.266316 & $\mathrm{H}$ & 2.660361 & 0.999834 & -1.382795 \\
\hline $\mathrm{H}$ & -3.039951 & -1.743934 & -1.560132 & $\mathrm{H}$ & 3.648309 & 5.620592 & -0.093295 \\
\hline $\mathrm{H}$ & -1.888919 & -0.616304 & -2.263571 & $\mathrm{H}$ & 1.950895 & 5.915031 & -0.550058 \\
\hline $\mathrm{H}$ & -4.312507 & 0.198089 & 2.713469 & $\mathrm{H}$ & 2.475392 & 6.168168 & 1.139971 \\
\hline $\mathrm{H}$ & -2.995372 & 1.224331 & 2.186985 & \multicolumn{4}{|c|}{$\omega$ B97X Energy = -1938.58789692 a.u. } \\
\hline $\mathrm{H}$ & -2.133765 & -0.436397 & 3.771358 & & & & \\
\hline $\mathrm{H}$ & -2.870715 & -1.745805 & 2.849831 & \multirow{2}{*}{\multicolumn{4}{|c|}{$(3 S, 5 R, 9 S, 10 R, 13 R, 24 R)-\mathbf{1}$, Conf M }} \\
\hline $\mathrm{H}$ & -1.459387 & 1.560844 & 0.713827 & & & & \\
\hline $\mathrm{H}$ & -0.668913 & 0.985846 & -0.751443 & $\mathrm{C}$ & -5.358843 & -1.957386 & 0.643586 \\
\hline $\mathrm{H}$ & -2.183656 & 1.859804 & -0.860273 & $\mathrm{C}$ & -6.427291 & -0.902530 & 0.869503 \\
\hline $\mathrm{H}$ & -4.069846 & 2.497042 & -0.686087 & $\mathrm{C}$ & -6.299003 & 0.309379 & -0.081573 \\
\hline $\mathrm{H}$ & -4.409571 & 2.488054 & 1.040218 & $\mathrm{C}$ & -4.838095 & 0.844487 & 0.025599 \\
\hline $\mathrm{H}$ & -5.730926 & 2.595534 & -0.109688 & $\mathrm{C}$ & -3.684274 & -0.193971 & -0.153959 \\
\hline $\mathrm{H}$ & -7.052274 & 0.593401 & 0.362248 & $\mathrm{C}$ & -3.956171 & -1.380413 & 0.786226 \\
\hline $\mathrm{H}$ & -6.156702 & 0.761417 & 1.868597 & $\mathrm{C}$ & -4.596316 & 2.107086 & -0.806794 \\
\hline $\mathrm{H}$ & -6.246009 & -0.815250 & 1.068821 & $\mathrm{C}$ & -3.257702 & 2.760456 & -0.459855 \\
\hline $\mathrm{H}$ & -5.959885 & 1.617881 & -2.230087 & $\mathrm{C}$ & -2.135621 & 1.764520 & -0.552746 \\
\hline $\mathrm{H}$ & -0.475513 & -1.776179 & -1.374058 & $\mathrm{C}$ & -2.369742 & 0.532042 & 0.296233 \\
\hline $\mathrm{H}$ & 0.539670 & -0.825009 & -0.328767 & $\mathrm{C}$ & -3.512746 & -0.707864 & -1.589615 \\
\hline $\mathrm{H}$ & 2.757332 & -3.556437 & -2.213017 & $\mathrm{H}$ & -4.752186 & 1.156548 & 1.077588 \\
\hline $\mathrm{H}$ & 1.537288 & -2.286866 & -2.270067 & $\mathrm{C}$ & -6.738093 & -0.061541 & -1.504265 \\
\hline $\mathrm{H}$ & 2.799402 & -2.550958 & 1.730137 & $\mathrm{C}$ & -7.264819 & 1.390334 & 0.428641 \\
\hline $\mathrm{H}$ & 0.951862 & -0.761403 & 1.812127 & 0 & -7.729989 & -1.485902 & 0.839986 \\
\hline
\end{tabular}




\begin{tabular}{|c|c|c|c|c|c|c|c|}
\hline $\mathrm{C}$ & -1.148781 & -0.378965 & 0.398323 & $\mathrm{H}$ & -2.583955 & -1.277130 & -1.677370 \\
\hline $\mathrm{C}$ & -0.040772 & 0.192372 & 1.244536 & $\mathrm{H}$ & -4.317793 & -1.377460 & -1.885741 \\
\hline $\mathrm{C}$ & 1.395553 & -0.312778 & 1.027311 & $\mathrm{H}$ & -3.466574 & 0.100748 & -2.319576 \\
\hline 0 & -0.222447 & 1.024038 & 2.106048 & $\mathrm{H}$ & -6.507201 & 0.742018 & -2.205824 \\
\hline $\mathrm{C}$ & 1.532904 & -1.833094 & 0.862754 & $\mathrm{H}$ & -7.820878 & -0.206762 & -1.533068 \\
\hline $\mathrm{C}$ & 2.292326 & -2.023922 & -0.435097 & $\mathrm{H}$ & -6.272862 & -0.971440 & -1.882887 \\
\hline $\mathrm{C}$ & 1.965476 & 0.244434 & -0.267333 & $\mathrm{H}$ & -8.250185 & 0.951527 & 0.594425 \\
\hline $\mathrm{C}$ & 2.515729 & -0.684268 & -1.041927 & $\mathrm{H}$ & -7.383030 & 2.202741 & -0.290498 \\
\hline $\mathrm{C}$ & -1.087918 & 1.963957 & -1.344382 & $\mathrm{H}$ & -6.917096 & 1.818518 & 1.373346 \\
\hline 0 & 2.637727 & -3.081517 & -0.908192 & $\mathrm{H}$ & -7.832190 & -1.954784 & 0.006520 \\
\hline 0 & 2.202016 & 0.139066 & 2.099846 & $\mathrm{H}$ & -1.422260 & -1.312123 & 0.900484 \\
\hline $\mathrm{C}$ & 3.331296 & -0.515077 & -2.276125 & $\mathrm{H}$ & -0.751236 & -0.671892 & -0.575881 \\
\hline S & 4.990818 & 0.188707 & -1.985402 & $\mathrm{H}$ & 2.107322 & -2.246463 & 1.690538 \\
\hline $\mathrm{C}$ & 5.829266 & -1.101117 & -1.008138 & $\mathrm{H}$ & 0.583718 & -2.366703 & 0.807056 \\
\hline C & 5.850003 & -0.886927 & 0.507959 & $\mathrm{H}$ & 2.002814 & 1.316272 & -0.431377 \\
\hline $\mathrm{C}$ & 6.262718 & 0.532879 & 0.864380 & $\mathrm{H}$ & -0.282137 & 1.246995 & -1.452539 \\
\hline 0 & 4.624040 & -1.196430 & 1.117846 & $\mathrm{H}$ & -1.004802 & 2.872287 & -1.931835 \\
\hline 0 & 7.431016 & 0.861980 & 0.335789 & $\mathrm{H}$ & 1.699731 & 0.837136 & 2.547947 \\
\hline 0 & 5.587742 & 1.261578 & 1.550390 & $\mathrm{H}$ & 2.860045 & 0.185916 & -2.965376 \\
\hline $\mathrm{C}$ & 7.897675 & 2.197140 & 0.596956 & $\mathrm{H}$ & 3.439639 & -1.473424 & -2.784634 \\
\hline $\mathrm{H}$ & -2.564989 & 0.893695 & 1.312789 & $\mathrm{H}$ & 5.369193 & -2.066683 & -1.221191 \\
\hline $\mathrm{H}$ & -5.490804 & -2.411839 & -0.344441 & $\mathrm{H}$ & 6.855953 & -1.132280 & -1.371790 \\
\hline $\mathrm{H}$ & -5.502629 & -2.758563 & 1.372738 & $\mathrm{H}$ & 6.618579 & -1.556240 & 0.912339 \\
\hline $\mathrm{H}$ & -6.322482 & -0.521925 & 1.891972 & $\mathrm{H}$ & 4.240176 & -0.393680 & 1.505895 \\
\hline $\mathrm{H}$ & -3.813918 & -1.051302 & 1.823193 & $\mathrm{H}$ & 7.186374 & 2.923474 & 0.205556 \\
\hline $\mathrm{H}$ & -3.232943 & -2.180066 & 0.607023 & $\mathrm{H}$ & 8.026677 & 2.345700 & 1.668170 \\
\hline $\mathrm{H}$ & -5.393680 & 2.831026 & -0.632693 & $\mathrm{H}$ & 8.850249 & 2.279909 & 0.082953 \\
\hline $\mathrm{H}$ & -4.615145 & 1.874463 & -1.875286 & \multirow{2}{*}{\multicolumn{4}{|c|}{$\omega \mathrm{B} 97 \mathrm{X}$ Energy = -1938.58783425 a.u. }} \\
\hline $\mathrm{H}$ & -3.065506 & 3.613211 & -1.114403 & & & & \\
\hline $\mathrm{H}$ & -3.308474 & 3.141369 & 0.567417 & \multicolumn{4}{|c|}{$(3 S, 5 R, 9 S, 10 R, 13 R, 24 R)-\mathbf{1}$, Conf $\mathrm{N}$} \\
\hline
\end{tabular}




\begin{tabular}{|c|c|c|c|c|c|c|c|}
\hline & & & & $\mathrm{C}$ & -4.323016 & -2.838257 & -0.336005 \\
\hline $\mathrm{C}$ & 4.196966 & 0.976967 & -1.857827 & $\mathrm{C}$ & -3.046164 & -3.177022 & 0.422046 \\
\hline $\mathrm{C}$ & 5.279901 & 0.390061 & -0.969659 & 0 & -5.162523 & -2.075314 & 0.498949 \\
\hline $\mathrm{C}$ & 4.789775 & -0.805006 & -0.120868 & 0 & -2.134993 & -3.758857 & -0.336680 \\
\hline $\mathrm{C}$ & 3.507838 & -0.352881 & 0.643617 & 0 & -2.918387 & -2.945150 & 1.601448 \\
\hline $\mathrm{C}$ & 2.368247 & 0.305978 & -0.197776 & $\mathrm{C}$ & -0.887222 & -4.087772 & 0.301471 \\
\hline $\mathrm{C}$ & 2.986824 & 1.427327 & -1.049138 & $\mathrm{H}$ & 1.967061 & 1.597597 & 1.465216 \\
\hline $\mathrm{C}$ & 2.960960 & -1.424272 & 1.591421 & $\mathrm{H}$ & 3.901179 & 0.242894 & -2.614986 \\
\hline $\mathrm{C}$ & 1.897992 & -0.850614 & 2.529595 & $\mathrm{H}$ & 4.615836 & 1.828438 & -2.399616 \\
\hline $\mathrm{C}$ & 0.822730 & -0.146372 & 1.749780 & $\mathrm{H}$ & 5.611138 & 1.168176 & -0.272526 \\
\hline $\mathrm{C}$ & 1.366884 & 0.931064 & 0.834118 & $\mathrm{H}$ & 3.290690 & 2.248217 & -0.387480 \\
\hline $\mathrm{C}$ & 1.612651 & -0.671724 & -1.107899 & $\mathrm{H}$ & 2.242671 & 1.835254 & -1.737919 \\
\hline $\mathrm{H}$ & 3.866082 & 0.463578 & 1.289004 & $\mathrm{H}$ & 3.769344 & -1.842955 & 2.192558 \\
\hline $\mathrm{C}$ & 4.606526 & -2.054852 & -0.993265 & $\mathrm{H}$ & 2.529945 & -2.256058 & 1.026547 \\
\hline $\mathrm{C}$ & 5.891926 & -1.118911 & 0.902764 & $\mathrm{H}$ & 1.464320 & -1.640009 & 3.147291 \\
\hline 0 & 6.445994 & 0.076573 & -1.731585 & $\mathrm{H}$ & 2.376617 & -0.131466 & 3.205336 \\
\hline $\mathrm{C}$ & 0.282602 & 1.800759 & 0.200062 & $\mathrm{H}$ & 0.753676 & -0.175982 & -1.567903 \\
\hline $\mathrm{C}$ & -0.429461 & 2.697549 & 1.180005 & $\mathrm{H}$ & 2.233982 & -1.039391 & -1.921717 \\
\hline $\mathrm{C}$ & -1.839111 & 3.233405 & 0.822926 & $\mathrm{H}$ & 1.231392 & -1.536228 & -0.563081 \\
\hline 0 & 0.059445 & 3.063312 & 2.224783 & $\mathrm{H}$ & 4.040351 & -1.872402 & -1.906751 \\
\hline $\mathrm{C}$ & -1.899661 & 3.805729 & -0.605302 & $\mathrm{H}$ & 4.095025 & -2.845545 & -0.441496 \\
\hline $\mathrm{C}$ & -2.651119 & 2.766909 & -1.414114 & $\mathrm{H}$ & 5.582405 & -2.451448 & -1.284433 \\
\hline $\mathrm{C}$ & -2.813274 & 2.074806 & 0.769703 & $\mathrm{H}$ & 6.854293 & -1.194314 & 0.393907 \\
\hline $\mathrm{C}$ & -3.230190 & 1.783039 & -0.460944 & $\mathrm{H}$ & 5.715359 & -2.067209 & 1.413304 \\
\hline $\mathrm{C}$ & -0.453614 & -0.501988 & 1.841186 & $\mathrm{H}$ & 5.965715 & -0.332500 & 1.659585 \\
\hline 0 & -2.769921 & 2.746929 & -2.617474 & $\mathrm{H}$ & 6.189963 & -0.517620 & -2.443174 \\
\hline 0 & -2.219176 & 4.176328 & 1.791407 & $\mathrm{H}$ & 0.734116 & 2.487064 & -0.522916 \\
\hline $\mathrm{C}$ & -4.069309 & 0.633362 & -0.900656 & $\mathrm{H}$ & -0.445689 & 1.211577 & -0.359695 \\
\hline$S$ & -2.931948 & -0.679928 & -1.488672 & $\mathrm{H}$ & -2.496440 & 4.719010 & -0.572357 \\
\hline $\mathrm{C}$ & -4.046991 & -2.104271 & -1.641086 & $\mathrm{H}$ & -0.940663 & 4.047638 & -1.062084 \\
\hline
\end{tabular}




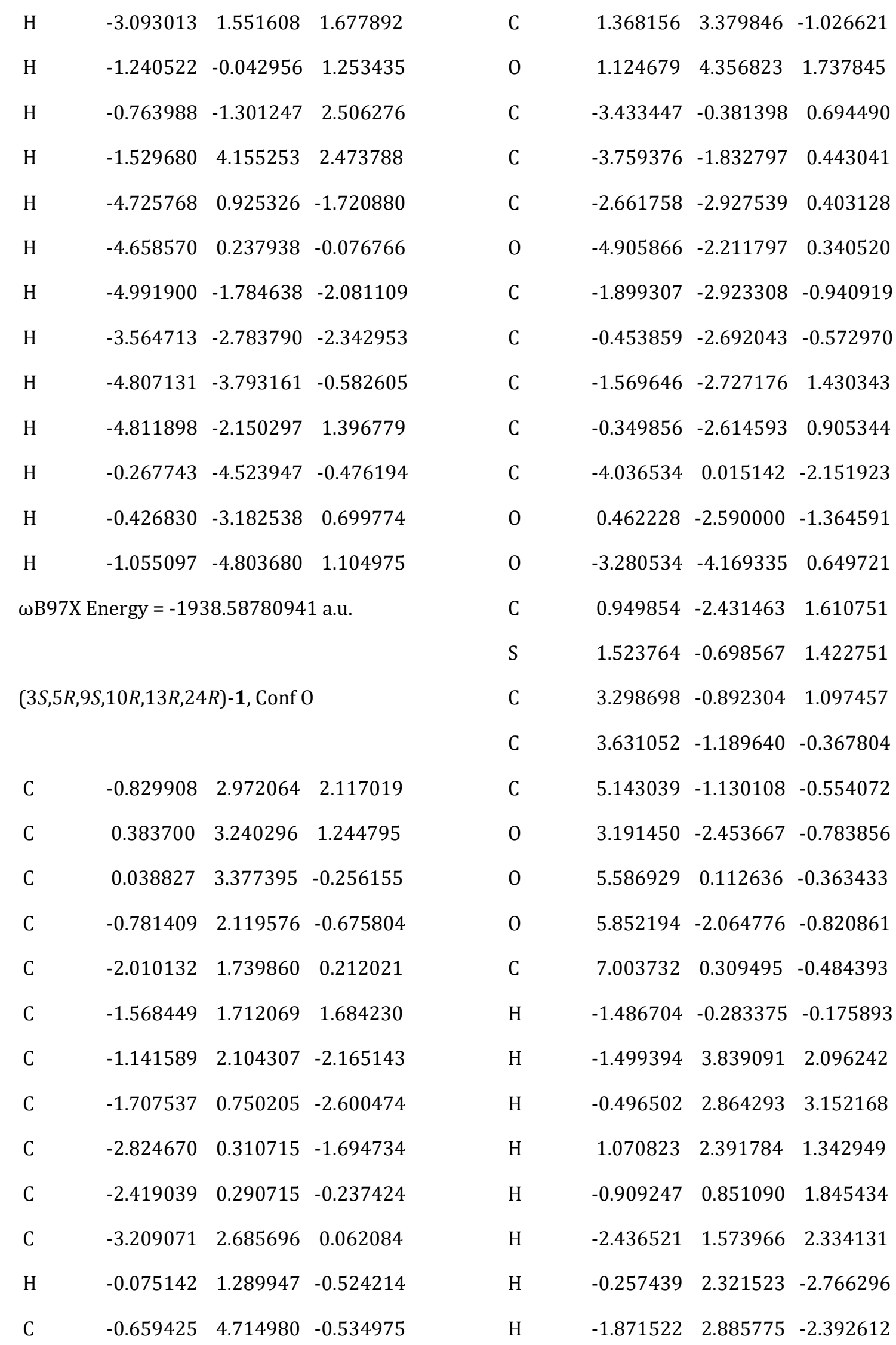




\begin{tabular}{|c|c|c|c|c|c|c|c|}
\hline $\mathrm{H}$ & -2.051179 & 0.792652 & -3.636056 & \multirow{2}{*}{\multicolumn{4}{|c|}{$(3 S, 5 R, 9 S, 10 R, 13 R, 24 R)-\mathbf{1}$, Conf $\mathrm{P}$}} \\
\hline $\mathrm{H}$ & -0.903649 & 0.003376 & -2.555126 & & & & \\
\hline $\mathrm{H}$ & -3.020702 & 3.653186 & 0.523745 & & & & \\
\hline $\mathrm{H}$ & -3.478522 & 2.860035 & -0.979384 & $\mathrm{C}$ & -3.984677 & -0.918224 & 2.286809 \\
\hline $\mathrm{H}$ & -4.087744 & 2.267701 & 0.559062 & $\mathrm{C}$ & -5.232190 & -1.217470 & 1.474426 \\
\hline $\mathrm{H}$ & -1.030053 & 4.756445 & -1.560628 & $\mathrm{C}$ & -4.923461 & -1.682857 & 0.033097 \\
\hline $\mathrm{H}$ & 0.052291 & 5.536455 & -0.421396 & $\mathrm{C}$ & -3.973777 & -0.628709 & -0.614115 \\
\hline $\mathrm{H}$ & -1.502112 & 4.913001 & 0.127630 & $\mathrm{C}$ & -2.690936 & -0.236437 & 0.187121 \\
\hline $\mathrm{H}$ & 2.045430 & 4.114638 & -0.588144 & $\mathrm{C}$ & -3.112627 & 0.137071 & 1.618178 \\
\hline $\mathrm{H}$ & 1.231390 & 3.642329 & -2.077035 & $\mathrm{C}$ & -3.653961 & -0.927736 & -2.081869 \\
\hline $\mathrm{H}$ & 1.852354 & 2.399086 & -0.979924 & $\mathrm{C}$ & -2.969031 & 0.260033 & -2.759409 \\
\hline $\mathrm{H}$ & 0.526424 & 5.106043 & 1.813157 & $\mathrm{C}$ & -1.770748 & 0.706314 & -1.969082 \\
\hline $\mathrm{H}$ & -3.042956 & -0.356231 & 1.716958 & $\mathrm{C}$ & -2.102528 & 1.028977 & -0.526767 \\
\hline $\mathrm{H}$ & -4.387475 & 0.146368 & 0.702655 & $\mathrm{C}$ & -1.619494 & -1.334015 & 0.240302 \\
\hline $\mathrm{H}$ & -2.002753 & -3.904404 & -1.405539 & $\mathrm{H}$ & -4.574617 & 0.293600 & -0.619774 \\
\hline $\mathrm{H}$ & -2.235262 & -2.177048 & -1.662514 & $\mathrm{C}$ & -4.379622 & -3.118188 & 0.026701 \\
\hline $\mathrm{H}$ & -1.801110 & -2.730841 & 2.489953 & $\mathrm{C}$ & -6.253509 & -1.693782 & -0.735930 \\
\hline $\mathrm{H}$ & -4.851721 & -0.289150 & -1.505495 & 0 & -6.085665 & -2.125471 & 2.171183 \\
\hline $\mathrm{H}$ & -4.253159 & 0.075456 & -3.213354 & $\mathrm{C}$ & -0.956293 & 1.700454 & 0.226527 \\
\hline $\mathrm{H}$ & -4.228603 & -4.031748 & 0.496858 & $\mathrm{C}$ & -0.674880 & 3.107303 & -0.237106 \\
\hline $\mathrm{H}$ & 1.708562 & -3.083649 & 1.179464 & $\mathrm{C}$ & 0.717070 & 3.724924 & 0.039481 \\
\hline $\mathrm{H}$ & 0.846209 & -2.662528 & 2.669251 & 0 & -1.498968 & 3.805719 & -0.783085 \\
\hline $\mathrm{H}$ & 3.748365 & 0.057150 & 1.389177 & $\mathrm{C}$ & 1.172206 & 3.498878 & 1.492177 \\
\hline $\mathrm{H}$ & 3.703590 & -1.672458 & 1.744756 & $\mathrm{C}$ & 2.220584 & 2.408807 & 1.408717 \\
\hline $\mathrm{H}$ & 3.201824 & -0.395563 & -0.990413 & $\mathrm{C}$ & 1.764967 & 2.983459 & -0.765145 \\
\hline $\mathrm{H}$ & 2.241052 & -2.410056 & -0.989730 & $\mathrm{C}$ & 2.569343 & 2.222177 & -0.024734 \\
\hline $\mathrm{H}$ & 7.170704 & 1.365312 & -0.293356 & $\mathrm{C}$ & -0.556175 & 0.752556 & -2.504393 \\
\hline $\mathrm{H}$ & 7.533847 & -0.299017 & 0.247516 & 0 & 2.699463 & 1.809754 & 2.345334 \\
\hline $\mathrm{H}$ & 7.335472 & 0.047397 & -1.488541 & 0 & 0.680351 & 5.087072 & -0.300603 \\
\hline & $\operatorname{ergy}=-1 c$ & 8.587752 & 1 a.u. & $\mathrm{C}$ & 3.627240 & 1.278669 & -0.482971 \\
\hline
\end{tabular}




\begin{tabular}{|c|c|c|c|c|c|c|c|}
\hline$S$ & 3.023233 & -0.452496 & -0.500752 & $\mathrm{H}$ & 1.655555 & 4.416013 & 1.833855 \\
\hline $\mathrm{C}$ & 3.646617 & -1.106666 & 1.077080 & $\mathrm{H}$ & 0.387927 & 3.246911 & 2.205036 \\
\hline $\mathrm{C}$ & 5.092443 & -1.583547 & 1.034470 & $\mathrm{H}$ & 1.798909 & 3.085362 & -1.844615 \\
\hline $\mathrm{C}$ & 5.330071 & -2.609861 & -0.063260 & $\mathrm{H}$ & 0.326655 & 1.036407 & -1.942514 \\
\hline 0 & 5.979396 & -0.504628 & 0.856872 & $\mathrm{H}$ & -0.399629 & 0.490606 & -3.545536 \\
\hline 0 & 4.483775 & -3.622714 & -0.008971 & $\mathrm{H}$ & -0.180810 & 5.237361 & -0.721352 \\
\hline 0 & 6.218470 & -2.494833 & -0.874951 & $\mathrm{H}$ & 4.508705 & 1.311591 & 0.155124 \\
\hline $\mathrm{C}$ & 4.643595 & -4.645327 & -1.008787 & $\mathrm{H}$ & 3.930015 & 1.513521 & -1.502546 \\
\hline $\mathrm{H}$ & -2.919450 & 1.759848 & -0.555326 & $\mathrm{H}$ & 3.537010 & -0.339744 & 1.843350 \\
\hline $\mathrm{H}$ & -3.417680 & -1.841964 & 2.445473 & $\mathrm{H}$ & 2.996805 & -1.942948 & 1.333889 \\
\hline $\mathrm{H}$ & -4.290069 & -0.570320 & 3.276719 & $\mathrm{H}$ & 5.304766 & -2.085840 & 1.987520 \\
\hline $\mathrm{H}$ & -5.820242 & -0.295694 & 1.397739 & $\mathrm{H}$ & 6.582082 & -0.749167 & 0.141493 \\
\hline $\mathrm{H}$ & -3.664296 & 1.085076 & 1.589700 & $\mathrm{H}$ & 3.869110 & -5.377622 & -0.803349 \\
\hline $\mathrm{H}$ & -2.230699 & 0.303289 & 2.241999 & $\mathrm{H}$ & 5.630788 & -5.097746 & -0.925501 \\
\hline $\mathrm{H}$ & -4.570323 & -1.160357 & -2.626112 & $\mathrm{H}$ & 4.513470 & -4.217382 & -2.001884 \\
\hline $\mathrm{H}$ & -3.010163 & -1.807910 & -2.164786 & \multirow{2}{*}{\multicolumn{4}{|c|}{$\omega \mathrm{B} 97 \mathrm{X}$ Energy = -1938.58773136 a.u. }} \\
\hline $\mathrm{H}$ & -2.679680 & 0.006028 & -3.781347 & & & & \\
\hline $\mathrm{H}$ & -3.683455 & 1.090156 & -2.818292 & \multirow{2}{*}{\multicolumn{4}{|c|}{$(3 S, 5 R, 9 S, 10 R, 13 R, 24 R)-\mathbf{1}$, Conf $Q$}} \\
\hline $\mathrm{H}$ & -1.918076 & -2.167499 & 0.872912 & & & & \\
\hline $\mathrm{H}$ & -1.381585 & -1.732763 & -0.746190 & $\mathrm{C}$ & -3.608651 & 0.826549 & -1.909626 \\
\hline $\mathrm{H}$ & -0.692059 & -0.936819 & 0.661443 & $\mathrm{C}$ & -4.894660 & 0.436959 & -1.201360 \\
\hline $\mathrm{H}$ & -4.003500 & -3.389115 & -0.961353 & $\mathrm{C}$ & -4.743569 & 0.352269 & 0.334556 \\
\hline $\mathrm{H}$ & -5.181437 & -3.821353 & 0.265318 & $\mathrm{C}$ & -3.532240 & -0.580554 & 0.644680 \\
\hline $\mathrm{H}$ & -3.573498 & -3.283580 & 0.741406 & $\mathrm{C}$ & -2.187507 & -0.266678 & -0.084330 \\
\hline $\mathrm{H}$ & -7.004498 & -2.236804 & -0.159725 & $\mathrm{C}$ & -2.474004 & -0.138097 & -1.589797 \\
\hline $\mathrm{H}$ & -6.159895 & -2.188533 & -1.704146 & C & -3.319125 & -0.814883 & 2.143092 \\
\hline $\mathrm{H}$ & -6.622052 & -0.677916 & -0.905918 & C & -2.317100 & -1.942522 & 2.397093 \\
\hline $\mathrm{H}$ & -5.576825 & -2.912192 & 2.388078 & $\mathrm{C}$ & -1.036527 & -1.702322 & 1.647133 \\
\hline $\mathrm{H}$ & -1.217819 & 1.805866 & 1.283967 & $\mathrm{C}$ & -1.254556 & -1.502896 & 0.161991 \\
\hline $\mathrm{H}$ & -0.037092 & 1.111560 & 0.201951 & $\mathrm{C}$ & -1.485207 & 1.003171 & 0.414168 \\
\hline
\end{tabular}




\begin{tabular}{|c|c|c|c|c|c|c|c|}
\hline $\mathrm{H}$ & -3.839869 & -1.552043 & 0.228817 & $\mathrm{H}$ & -4.265147 & -1.072445 & 2.621465 \\
\hline $\mathrm{C}$ & -4.644649 & 1.755498 & 0.949604 & $\mathrm{H}$ & -2.962452 & 0.097728 & 2.628952 \\
\hline $\mathrm{C}$ & -6.022920 & -0.297871 & 0.883753 & $\mathrm{H}$ & -2.119240 & -2.047242 & 3.465837 \\
\hline 0 & -5.970472 & 1.287308 & -1.598382 & $\mathrm{H}$ & -2.755953 & -2.886492 & 2.051746 \\
\hline $\mathrm{C}$ & 0.037371 & -1.482242 & -0.651141 & $\mathrm{H}$ & -2.033959 & 1.906199 & 0.153869 \\
\hline $\mathrm{C}$ & 0.733327 & -2.816623 & -0.719770 & $\mathrm{H}$ & -1.340053 & 1.002204 & 1.494922 \\
\hline C & 2.247605 & -2.861921 & -1.032126 & $\mathrm{H}$ & -0.497968 & 1.093852 & -0.046325 \\
\hline 0 & 0.157577 & -3.872289 & -0.584475 & $\mathrm{H}$ & -3.935297 & 2.408406 & 0.440826 \\
\hline $\mathrm{C}$ & 2.651149 & -1.923585 & -2.182642 & $\mathrm{H}$ & -4.350448 & 1.703165 & 1.999120 \\
\hline $\mathrm{C}$ & 3.438033 & -0.807634 & -1.526463 & $\mathrm{H}$ & -5.621496 & 2.244213 & 0.917348 \\
\hline $\mathrm{C}$ & 3.038314 & -2.325402 & 0.147528 & $\mathrm{H}$ & -6.897152 & 0.200641 & 0.461830 \\
\hline $\mathrm{C}$ & 3.691692 & -1.194354 & -0.112738 & $\mathrm{H}$ & -6.088398 & -0.212869 & 1.969853 \\
\hline $\mathrm{C}$ & 0.135584 & -1.632970 & 2.267941 & $\mathrm{H}$ & -6.074256 & -1.358478 & 0.620664 \\
\hline 0 & 3.805061 & 0.216299 & -2.057069 & $\mathrm{H}$ & -5.718174 & 2.198009 & -1.419730 \\
\hline 0 & 2.608472 & -4.195287 & -1.294647 & $\mathrm{H}$ & -0.184835 & -1.234384 & -1.693443 \\
\hline $\mathrm{C}$ & 4.573851 & -0.383112 & 0.778348 & $\mathrm{H}$ & 0.738296 & -0.719803 & -0.308163 \\
\hline S & 3.713678 & 0.465915 & 2.143219 & $\mathrm{H}$ & 3.319443 & -2.474490 & -2.846459 \\
\hline $\mathrm{C}$ & 2.531082 & 1.501812 & 1.243863 & $\mathrm{H}$ & 1.832128 & -1.530067 & -2.783639 \\
\hline $\mathrm{C}$ & 3.156588 & 2.605661 & 0.389689 & $\mathrm{H}$ & 3.049271 & -2.867330 & 1.087315 \\
\hline $\mathrm{C}$ & 2.054343 & 3.383115 & -0.316868 & $\mathrm{H}$ & 1.071167 & -1.430549 & 1.758088 \\
\hline 0 & 3.931446 & 3.492340 & 1.155960 & $\mathrm{H}$ & 0.201491 & -1.772829 & 3.341924 \\
\hline 0 & 1.337969 & 2.630611 & -1.134862 & $\mathrm{H}$ & 1.841958 & -4.736676 & -1.048438 \\
\hline 0 & 1.869108 & 4.561861 & -0.124799 & $\mathrm{H}$ & 5.126398 & 0.343728 & 0.182516 \\
\hline $\mathrm{C}$ & 0.269898 & 3.286903 & -1.842581 & $\mathrm{H}$ & 5.302363 & -1.022299 & 1.277395 \\
\hline $\mathrm{H}$ & -1.819557 & -2.375782 & -0.186245 & $\mathrm{H}$ & 1.895948 & 0.872374 & 0.619129 \\
\hline $\mathrm{H}$ & -3.330061 & 1.850811 & -1.637700 & $\mathrm{H}$ & 1.898667 & 1.946586 & 2.015144 \\
\hline $\mathrm{H}$ & -3.793650 & 0.833747 & -2.986563 & $\mathrm{H}$ & 3.778828 & 2.151806 & -0.389583 \\
\hline $\mathrm{H}$ & -5.190886 & -0.558010 & -1.553103 & $\mathrm{H}$ & 3.478859 & 4.346631 & 1.141299 \\
\hline $\mathrm{H}$ & -2.728451 & -1.127826 & -1.989115 & $\mathrm{H}$ & -0.452991 & 3.693319 & -1.135981 \\
\hline $\mathrm{H}$ & -1.577297 & 0.193583 & -2.120339 & $\mathrm{H}$ & 0.670645 & 4.084724 & -2.465993 \\
\hline
\end{tabular}




\begin{tabular}{|c|c|c|c|c|c|c|c|}
\hline $\mathrm{H}$ & -0.188745 & 2.516889 & -2.456522 & 0 & 3.767431 & -3.480627 & -1.080583 \\
\hline & nergy $=-193$ & 38.5876655 & 0 a.u. & $\mathrm{C}$ & -0.545564 & -2.992005 & 0.593315 \\
\hline & & & & S & -1.417871 & -1.390583 & 0.742105 \\
\hline & $, 10 R, 13 R, 24$ & $4 R$ )-1, Conf $\mathrm{R}$ & & $\mathrm{C}$ & -2.968668 & -1.862427 & 1.556229 \\
\hline & & & & $\mathrm{C}$ & -4.012847 & -2.472907 & 0.633795 \\
\hline $\mathrm{C}$ & -1.212341 & 2.324735 & -1.714698 & $\mathrm{C}$ & -4.266853 & -1.619951 & -0.601565 \\
\hline $\mathrm{C}$ & -1.839233 & 2.558218 & -0.351758 & 0 & -3.624668 & -3.765047 & 0.232241 \\
\hline $\mathrm{C}$ & -0.858672 & 3.172695 & 0.674065 & 0 & -4.476296 & -0.348411 & -0.314526 \\
\hline $\mathrm{C}$ & 0.424333 & 2.287007 & 0.704599 & 0 & -4.285111 & -2.091350 & -1.714733 \\
\hline $\mathrm{C}$ & 1.085995 & 1.935609 & -0.667079 & $\mathrm{C}$ & -4.709144 & 0.535183 & -1.428290 \\
\hline $\mathrm{C}$ & 0.002785 & 1.410225 & -1.623572 & $\mathrm{H}$ & 1.526091 & 0.037458 & 0.193317 \\
\hline $\mathrm{C}$ & 1.467195 & 2.772189 & 1.717738 & $\mathrm{H}$ & -0.943466 & 3.284573 & -2.169231 \\
\hline $\mathrm{C}$ & 2.583934 & 1.744738 & 1.919686 & $\mathrm{H}$ & -1.962325 & 1.874960 & -2.370806 \\
\hline $\mathrm{C}$ & 3.163783 & 1.302006 & 0.604511 & $\mathrm{H}$ & -2.162312 & 1.588613 & 0.047495 \\
\hline $\mathrm{C}$ & 2.111840 & 0.786641 & -0.352913 & $\mathrm{H}$ & -0.330691 & 0.422637 & -1.284008 \\
\hline C & 1.815401 & 3.110270 & -1.333107 & $\mathrm{H}$ & 0.420083 & 1.278750 & -2.625451 \\
\hline $\mathrm{H}$ & 0.060575 & 1.320269 & 1.082041 & $\mathrm{H}$ & 0.993341 & 2.964285 & 2.681521 \\
\hline $\mathrm{C}$ & -0.598758 & 4.652584 & 0.362925 & $\mathrm{H}$ & 1.906858 & 3.719051 & 1.393533 \\
\hline $\mathrm{C}$ & -1.541435 & 3.102777 & 2.048623 & $\mathrm{H}$ & 3.366636 & 2.149661 & 2.564484 \\
\hline 0 & -3.046511 & 3.313142 & -0.470010 & $\mathrm{H}$ & 2.163787 & 0.868329 & 2.430817 \\
\hline $\mathrm{C}$ & 2.673205 & 0.103333 & -1.604247 & $\mathrm{H}$ & 1.118735 & 3.854790 & -1.714029 \\
\hline $\mathrm{C}$ & 3.532130 & -1.122615 & -1.412913 & $\mathrm{H}$ & 2.505948 & 3.614907 & -0.657879 \\
\hline $\mathrm{C}$ & 3.041163 & -2.360418 & -0.621702 & $\mathrm{H}$ & 2.396575 & 2.758847 & -2.189007 \\
\hline 0 & 4.619342 & -1.214438 & -1.941783 & $\mathrm{H}$ & 0.187326 & 5.052860 & 1.005417 \\
\hline $\mathrm{C}$ & 3.204171 & -2.157694 & 0.901721 & $\mathrm{H}$ & -1.499325 & 5.238979 & 0.561482 \\
\hline $\mathrm{C}$ & 1.829139 & -2.408409 & 1.484633 & $\mathrm{H}$ & -0.305331 & 4.836819 & -0.670621 \\
\hline $\mathrm{C}$ & 1.567277 & -2.660481 & -0.787989 & $\mathrm{H}$ & -2.542405 & 3.532700 & 1.983371 \\
\hline C & 0.898747 & -2.709508 & 0.362437 & $\mathrm{H}$ & -0.991499 & 3.663054 & 2.806601 \\
\hline $\mathrm{C}$ & 4.459829 & 1.399480 & 0.329589 & $\mathrm{H}$ & -1.635607 & 2.067271 & 2.390253 \\
\hline 0 & 1.536242 & -2.373689 & 2.657271 & $\mathrm{H}$ & -2.852440 & 4.123361 & -0.950511 \\
\hline
\end{tabular}




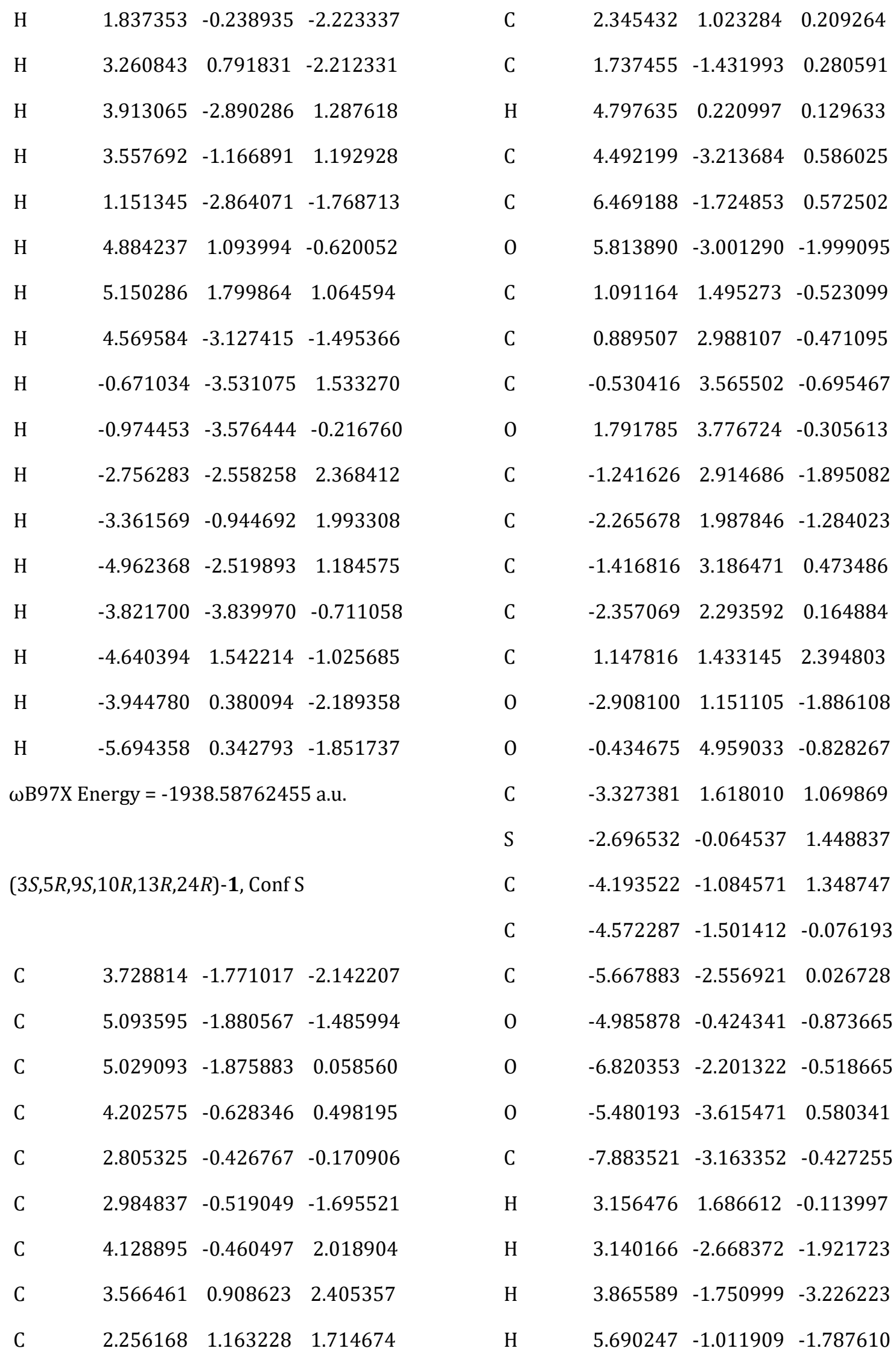




\begin{tabular}{|c|c|c|c|c|c|c|c|}
\hline $\mathrm{H}$ & 3.538455 & 0.362756 & -2.041737 & $\mathrm{H}$ & -8.736554 & -2.702919 & -0.916600 \\
\hline $\mathrm{H}$ & 2.012647 & -0.497321 & -2.194449 & $\mathrm{H}$ & -7.602582 & -4.084787 & -0.935938 \\
\hline $\mathrm{H}$ & 5.121784 & -0.567965 & 2.458019 & $\mathrm{H}$ & -8.110279 & -3.375781 & 0.616902 \\
\hline $\mathrm{H}$ & 3.506236 & -1.242472 & 2.462665 & \multicolumn{4}{|c|}{$\omega B 97 X$ Energy = -1938.58754343 a.u. } \\
\hline $\mathrm{H}$ & 3.446852 & 0.983901 & 3.488167 & & & & \\
\hline $\mathrm{H}$ & 4.282691 & 1.681283 & 2.100657 & \multicolumn{4}{|c|}{$(3 S, 5 R, 9 S, 10 R, 13 R, 24 R)-\mathbf{1}$, Conf $\mathrm{T}$} \\
\hline $\mathrm{H}$ & 1.936107 & -2.435455 & -0.089997 & & & & \\
\hline $\mathrm{H}$ & 1.649594 & -1.489071 & 1.365812 & $\mathrm{C}$ & 3.670797 & 0.846880 & 2.202192 \\
\hline $\mathrm{H}$ & 0.757592 & -1.142615 & -0.108859 & $\mathrm{C}$ & 4.989586 & 0.812363 & 1.459600 \\
\hline $\mathrm{H}$ & 3.581974 & -3.546332 & 0.087091 & $\mathrm{C}$ & 4.841510 & 1.075852 & -0.057002 \\
\hline $\mathrm{H}$ & 4.280150 & -3.155270 & 1.655004 & $\mathrm{C}$ & 3.772132 & 0.081682 & -0.610910 \\
\hline $\mathrm{H}$ & 5.245446 & -3.994291 & 0.453692 & $\mathrm{C}$ & 2.407532 & 0.004453 & 0.145554 \\
\hline $\mathrm{H}$ & 7.114187 & -2.448843 & 0.071680 & C & 2.693382 & -0.178868 & 1.645416 \\
\hline $\mathrm{H}$ & 6.541346 & -1.907874 & 1.646022 & $\mathrm{C}$ & 3.559535 & 0.200276 & -2.122368 \\
\hline $\mathrm{H}$ & 6.859302 & -0.722983 & 0.370545 & $\mathrm{C}$ & 2.747527 & -0.977449 & -2.660714 \\
\hline $\mathrm{H}$ & 5.271995 & -3.787227 & -1.882304 & C & 1.464942 & -1.157824 & -1.896887 \\
\hline $\mathrm{H}$ & 1.177461 & 1.267165 & -1.589835 & $\mathrm{C}$ & 1.670162 & -1.269023 & -0.399747 \\
\hline $\mathrm{H}$ & 0.189354 & 0.984407 & -0.179684 & C & 1.506541 & 1.231426 & -0.054336 \\
\hline $\mathrm{H}$ & -1.770610 & 3.702910 & -2.434316 & $\mathrm{H}$ & 4.225261 & -0.907777 & -0.446017 \\
\hline $\mathrm{H}$ & -0.602066 & 2.392393 & -2.605488 & C & 4.544771 & 2.555455 & -0.330194 \\
\hline $\mathrm{H}$ & -1.251622 & 3.625618 & 1.451441 & $\mathrm{C}$ & 6.191575 & 0.749809 & -0.717597 \\
\hline $\mathrm{H}$ & 0.185773 & 1.587928 & 1.919825 & 0 & 5.849460 & 1.750560 & 2.106522 \\
\hline $\mathrm{H}$ & 1.164776 & 1.501036 & 3.477410 & $\mathrm{C}$ & 0.399732 & -1.617505 & 0.373946 \\
\hline $\mathrm{H}$ & 0.481053 & 5.190809 & -0.606836 & $\mathrm{C}$ & -0.142071 & -2.989834 & 0.074777 \\
\hline $\mathrm{H}$ & -4.298603 & 1.514973 & 0.588178 & $\mathrm{C}$ & -1.626239 & -3.292930 & 0.389634 \\
\hline $\mathrm{H}$ & -3.446233 & 2.175288 & 1.997153 & 0 & 0.537268 & -3.896261 & -0.352188 \\
\hline $\mathrm{H}$ & -3.977172 & -1.975784 & 1.938240 & $\mathrm{C}$ & -1.985013 & -2.912788 & 1.839653 \\
\hline $\mathrm{H}$ & -5.022810 & -0.556548 & 1.823117 & $\mathrm{C}$ & -2.882973 & -1.701571 & 1.718243 \\
\hline $\mathrm{H}$ & -3.715126 & -2.016595 & -0.525343 & $\mathrm{C}$ & -2.552532 & -2.421216 & -0.436401 \\
\hline $\mathrm{H}$ & -4.202482 & 0.047301 & -1.207613 & $\mathrm{C}$ & -3.243640 & -1.540199 & 0.282986 \\
\hline
\end{tabular}




\begin{tabular}{|c|c|c|c|c|c|c|c|}
\hline $\mathrm{C}$ & 0.290423 & -1.193529 & -2.515221 & $\mathrm{H}$ & 6.422285 & -0.316813 & -0.646395 \\
\hline 0 & -3.248915 & -0.990237 & 2.626893 & $\mathrm{H}$ & 6.738634 & 1.648382 & 1.758614 \\
\hline 0 & -1.870672 & -4.650769 & 0.121139 & $\mathrm{H}$ & 0.623899 & -1.637800 & 1.444994 \\
\hline $\mathrm{C}$ & -4.271422 & -0.540555 & -0.130817 & $\mathrm{H}$ & -0.388999 & -0.871361 & 0.256456 \\
\hline $\mathrm{S}$ & -3.923023 & 0.446055 & -1.621065 & $\mathrm{H}$ & -2.564862 & -3.733648 & 2.265108 \\
\hline $\mathrm{C}$ & -2.351283 & 1.294990 & -1.277284 & $\mathrm{H}$ & -1.143960 & -2.717181 & 2.504221 \\
\hline $\mathrm{C}$ & -2.226845 & 2.053747 & 0.043398 & $\mathrm{H}$ & -2.620185 & -2.558977 & -1.510267 \\
\hline $\mathrm{C}$ & -3.477415 & 2.860791 & 0.350196 & $\mathrm{H}$ & -0.649824 & -1.326042 & -1.992307 \\
\hline 0 & -1.934934 & 1.181427 & 1.109189 & $\mathrm{H}$ & 0.225483 & -1.097394 & -3.593780 \\
\hline 0 & -3.681744 & 3.819813 & -0.534337 & $\mathrm{H}$ & -1.038510 & -5.011491 & -0.224104 \\
\hline 0 & -4.191580 & 2.624625 & 1.296005 & $\mathrm{H}$ & -4.493314 & 0.117023 & 0.709088 \\
\hline $\mathrm{C}$ & -4.880766 & 4.598348 & -0.369676 & $\mathrm{H}$ & -5.203599 & -1.052122 & -0.379986 \\
\hline $\mathrm{H}$ & 2.365527 & -2.103820 & -0.248177 & $\mathrm{H}$ & -1.517885 & 0.594709 & -1.334991 \\
\hline $\mathrm{H}$ & 3.249255 & 1.854954 & 2.153740 & $\mathrm{H}$ & -2.252259 & 1.993420 & -2.109354 \\
\hline $\mathrm{H}$ & 3.861365 & 0.640418 & 3.258070 & $\mathrm{H}$ & -1.394265 & 2.757475 & -0.062191 \\
\hline $\mathrm{H}$ & 5.416563 & -0.195777 & 1.572379 & $\mathrm{H}$ & -2.697230 & 1.140071 & 1.704011 \\
\hline $\mathrm{H}$ & 3.102822 & -1.183791 & 1.809088 & $\mathrm{H}$ & -4.868655 & 5.326506 & -1.174572 \\
\hline $\mathrm{H}$ & 1.763534 & -0.118820 & 2.216506 & $\mathrm{H}$ & -5.755077 & 3.953198 & -0.449087 \\
\hline $\mathrm{H}$ & 4.518257 & 0.227042 & -2.641734 & $\mathrm{H}$ & -4.873348 & 5.096644 & 0.598538 \\
\hline $\mathrm{H}$ & 3.046948 & 1.134986 & -2.365471 & \multicolumn{4}{|c|}{$\omega \mathrm{B} 97 \mathrm{X}$ Energy = -1938.58742560 a.u. } \\
\hline $\mathrm{H}$ & 2.539456 & -0.847271 & -3.724751 & & & & \\
\hline $\mathrm{H}$ & 3.345211 & -1.891241 & -2.555727 & \multicolumn{4}{|c|}{$(3 S, 5 R, 9 S, 10 R, 13 R, 24 R)-\mathbf{1}$, Conf $\mathrm{U}$} \\
\hline $\mathrm{H}$ & 1.945386 & 2.135720 & 0.361213 & & & & \\
\hline $\mathrm{H}$ & 1.291759 & 1.420779 & -1.107256 & $\mathrm{C}$ & -0.972294 & 2.423978 & -2.052557 \\
\hline $\mathrm{H}$ & 0.551321 & 1.081796 & 0.455728 & $\mathrm{C}$ & -1.962091 & 2.323772 & -0.904467 \\
\hline $\mathrm{H}$ & 3.735415 & 2.954121 & 0.279397 & $\mathrm{C}$ & -1.390343 & 2.840387 & 0.435809 \\
\hline $\mathrm{H}$ & 4.287131 & 2.714157 & -1.379279 & $\mathrm{C}$ & -0.054312 & 2.079712 & 0.697394 \\
\hline $\mathrm{H}$ & 5.431038 & 3.155058 & -0.115358 & $\mathrm{C}$ & 1.001113 & 2.079862 & -0.456865 \\
\hline $\mathrm{H}$ & 7.005481 & 1.304794 & -0.243018 & $\mathrm{C}$ & 0.310705 & 1.653838 & -1.763235 \\
\hline $\mathrm{H}$ & 6.207569 & 1.033233 & -1.770721 & $\mathrm{C}$ & 0.586866 & 2.430487 & 2.044410 \\
\hline
\end{tabular}




\begin{tabular}{|c|c|c|c|c|c|c|c|}
\hline $\mathrm{C}$ & 1.752426 & 1.498201 & 2.384591 & $\mathrm{H}$ & -1.444017 & 2.022321 & -2.952944 \\
\hline $\mathrm{C}$ & 2.727590 & 1.408407 & 1.243215 & $\mathrm{H}$ & -2.218084 & 1.266923 & -0.760325 \\
\hline $\mathrm{C}$ & 2.065443 & 0.994829 & -0.052055 & $\mathrm{H}$ & 0.070240 & 0.587152 & -1.704978 \\
\hline $\mathrm{C}$ & 1.694359 & 3.432619 & -0.669670 & $\mathrm{H}$ & 0.995958 & 1.784055 & -2.605322 \\
\hline $\mathrm{H}$ & -0.365124 & 1.026540 & 0.772669 & $\mathrm{H}$ & -0.156020 & 2.363003 & 2.840476 \\
\hline $\mathrm{C}$ & -1.260950 & 4.369239 & 0.426903 & $\mathrm{H}$ & 0.945610 & 3.463299 & 2.041463 \\
\hline $\mathrm{C}$ & -2.399684 & 2.476777 & 1.535192 & $\mathrm{H}$ & 2.257134 & 1.832377 & 3.293360 \\
\hline 0 & -3.202214 & 2.944801 & -1.246867 & $\mathrm{H}$ & 1.351941 & 0.495285 & 2.584452 \\
\hline $\mathrm{C}$ & 3.039108 & 0.637555 & -1.181067 & $\mathrm{H}$ & 2.038633 & 3.882813 & 0.261156 \\
\hline $\mathrm{C}$ & 4.021887 & -0.483630 & -0.941001 & $\mathrm{H}$ & 2.567781 & 3.315009 & -1.315161 \\
\hline $\mathrm{C}$ & 3.566810 & -1.910994 & -0.542214 & $\mathrm{H}$ & 1.038209 & 4.147359 & -1.163112 \\
\hline 0 & 5.208989 & -0.325480 & -1.130119 & $\mathrm{H}$ & -2.253395 & 4.826908 & 0.444130 \\
\hline $\mathrm{C}$ & 3.205559 & -1.992896 & 0.958115 & $\mathrm{H}$ & -0.732544 & 4.759542 & -0.442158 \\
\hline $\mathrm{C}$ & 1.759224 & -2.436868 & 0.993910 & $\mathrm{H}$ & -0.736180 & 4.720715 & 1.317043 \\
\hline $\mathrm{C}$ & 2.300600 & -2.358432 & -1.237188 & $\mathrm{H}$ & -3.396649 & 2.812096 & 1.243930 \\
\hline $\mathrm{C}$ & 1.305474 & -2.658293 & -0.404434 & $\mathrm{H}$ & -2.155807 & 2.953624 & 2.485973 \\
\hline $\mathrm{C}$ & 4.014066 & 1.710148 & 1.379705 & $\mathrm{H}$ & -2.443429 & 1.395757 & 1.697175 \\
\hline 0 & 1.088400 & -2.586564 & 1.991050 & $\mathrm{H}$ & -3.024561 & 3.849202 & -1.521889 \\
\hline 0 & 4.603056 & -2.805556 & -0.882212 & $\mathrm{H}$ & 2.461171 & 0.321332 & -2.055795 \\
\hline $\mathrm{C}$ & -0.074922 & -3.115531 & -0.734988 & $\mathrm{H}$ & 3.636625 & 1.497379 & -1.485649 \\
\hline S & -1.275142 & -1.736601 & -0.701953 & $\mathrm{H}$ & 3.831932 & -2.752062 & 1.427508 \\
\hline $\mathrm{C}$ & -1.943446 & -1.810747 & 0.989274 & $\mathrm{H}$ & 3.333702 & -1.063343 & 1.514650 \\
\hline $\mathrm{C}$ & -3.313071 & -2.466742 & 1.065429 & $\mathrm{H}$ & 2.268426 & -2.448212 & -2.317599 \\
\hline $\mathrm{C}$ & -4.297197 & -1.840804 & 0.086267 & $\mathrm{H}$ & 4.719454 & 1.663901 & 0.557865 \\
\hline 0 & -3.221261 & -3.848235 & 0.816374 & $\mathrm{H}$ & 4.407270 & 2.023630 & 2.341188 \\
\hline 0 & -4.272690 & -0.519722 & 0.119024 & $\mathrm{H}$ & 5.400898 & -2.264724 & -0.988569 \\
\hline 0 & -5.013647 & -2.503976 & -0.625949 & $\mathrm{H}$ & -0.418531 & -3.880563 & -0.039358 \\
\hline $\mathrm{C}$ & -5.144135 & 0.173401 & -0.794487 & $\mathrm{H}$ & -0.108199 & -3.529165 & -1.742021 \\
\hline $\mathrm{H}$ & 1.472330 & 0.097652 & 0.163292 & $\mathrm{H}$ & -1.236980 & -2.345068 & 1.623050 \\
\hline $\mathrm{H}$ & -0.755419 & 3.477497 & -2.261399 & $\mathrm{H}$ & -2.012745 & -0.786771 & 1.355865 \\
\hline
\end{tabular}




\begin{tabular}{|c|c|c|c|c|c|c|c|}
\hline $\mathrm{H}$ & -3.716278 & \multicolumn{2}{|c|}{$-2.291984 \quad 2.072338$} & $\mathrm{C}$ & 2.048112 & 0.872861 & 0.144712 \\
\hline $\mathrm{H}$ & -3.945107 & -4.078438 & 0.219071 & $\mathrm{C}$ & 3.354367 & 0.900782 & 0.386096 \\
\hline $\mathrm{H}$ & -4.877619 & 1.224148 & -0.710259 & $\mathrm{C}$ & -0.576934 & 2.523008 & 2.402792 \\
\hline $\mathrm{H}$ & -4.972494 & -0.181082 & -1.810319 & 0 & 5.176628 & 2.128700 & -0.589753 \\
\hline $\mathrm{H}$ & -6.183635 & 0.005703 & -0.514884 & 0 & 0.882550 & 1.005166 & -1.981896 \\
\hline \multicolumn{4}{|c|}{$\omega \mathrm{B} 97 \mathrm{X}$ Energy $=-1938.58741578$ a.u. } & $\mathrm{C}$ & 4.138848 & 0.157709 & 1.417267 \\
\hline \multirow{3}{*}{\multicolumn{4}{|c|}{$(3 S, 5 R, 9 S, 10 R, 13 R, 24 R)-\mathbf{1}$, Conf $\mathrm{V}$}} & $S$ & 3.406591 & -1.425491 & 1.934859 \\
\hline & & & & $\mathrm{C}$ & 4.171374 & -2.621662 & 0.801761 \\
\hline & & & & $\mathrm{C}$ & 3.784252 & -2.547205 & -0.668214 \\
\hline $\mathrm{C}$ & -4.346800 & 0.294871 & -1.891573 & $\mathrm{C}$ & 2.282346 & -2.484957 & -0.905925 \\
\hline $\mathrm{C}$ & -4.480881 & -1.082591 & -1.267272 & 0 & 4.418448 & -1.444378 & -1.269649 \\
\hline $\mathrm{C}$ & -4.344580 & -1.068385 & 0.272261 & 0 & 1.619493 & -3.402992 & -0.235039 \\
\hline $\mathrm{C}$ & -3.018528 & -0.328518 & 0.627423 & 0 & 1.773639 & -1.701624 & -1.679366 \\
\hline $\mathrm{C}$ & -2.785646 & 1.073333 & -0.021442 & $\mathrm{C}$ & 0.191458 & -3.433177 & -0.419363 \\
\hline $\mathrm{C}$ & -3.017302 & 0.947669 & -1.536254 & $\mathrm{H}$ & -0.725656 & 0.572070 & -0.168212 \\
\hline $\mathrm{C}$ & -2.720848 & -0.312302 & 2.129525 & $\mathrm{H}$ & -5.183135 & 0.927375 & -1.573957 \\
\hline $\mathrm{C}$ & -1.291606 & 0.152210 & 2.413347 & $\mathrm{H}$ & -4.431177 & 0.193739 & -2.976407 \\
\hline $\mathrm{C}$ & -1.001730 & 1.459437 & 1.728804 & $\mathrm{H}$ & -3.676557 & -1.716606 & -1.658173 \\
\hline $\mathrm{C}$ & -1.278905 & 1.424818 & 0.240581 & $\mathrm{H}$ & -2.202743 & 0.354626 & -1.970526 \\
\hline $\mathrm{C}$ & -3.676188 & 2.185768 & 0.545036 & $\mathrm{H}$ & -2.973160 & 1.932742 & -2.007161 \\
\hline $\mathrm{H}$ & -2.241438 & -0.962674 & 0.172597 & $\mathrm{H}$ & -2.856172 & -1.309103 & 2.551896 \\
\hline $\mathrm{C}$ & -5.601417 & -0.473943 & 0.923265 & $\mathrm{H}$ & -3.420727 & 0.344823 & 2.653049 \\
\hline $\mathrm{C}$ & -4.237353 & -2.530541 & 0.732375 & $\mathrm{H}$ & -1.121610 & 0.240235 & 3.488433 \\
\hline 0 & -5.680242 & -1.723315 & -1.702590 & $\mathrm{H}$ & -0.594374 & -0.609728 & 2.038118 \\
\hline $\mathrm{C}$ & -0.786360 & 2.667955 & -0.514734 & $\mathrm{H}$ & -3.604915 & 2.262675 & 1.630438 \\
\hline $\mathrm{C}$ & 0.710067 & 2.872137 & -0.488723 & $\mathrm{H}$ & -3.377723 & 3.151565 & 0.130218 \\
\hline $\mathrm{C}$ & 1.619749 & 1.742002 & -1.022853 & $\mathrm{H}$ & -4.724295 & 2.044337 & 0.289428 \\
\hline 0 & 1.214782 & 3.890138 & -0.073330 & $\mathrm{H}$ & -5.442821 & -0.293080 & 1.987737 \\
\hline $\mathrm{C}$ & 2.951770 & 2.286278 & -1.564704 & $\mathrm{H}$ & -6.431391 & -1.180123 & 0.840846 \\
\hline $\mathrm{C}$ & 4.006157 & 1.819612 & -0.582234 & $\mathrm{H}$ & -5.922528 & 0.465737 & 0.473580 \\
\hline
\end{tabular}




\begin{tabular}{|c|c|c|c|c|c|c|c|}
\hline $\mathrm{H}$ & -5.036882 & -3.116509 & 0.276046 & $\mathrm{C}$ & 2.804778 & -1.067130 & -1.548922 \\
\hline $\mathrm{H}$ & -4.336138 & -2.626806 & 1.814932 & $\mathrm{C}$ & 2.647277 & 0.237728 & 2.109562 \\
\hline $\mathrm{H}$ & -3.279993 & -2.970361 & 0.436262 & $\mathrm{C}$ & 1.231126 & -0.225598 & 2.453864 \\
\hline $\mathrm{H}$ & -6.421639 & -1.146693 & -1.495731 & $\mathrm{C}$ & 0.917439 & -1.542203 & 1.798160 \\
\hline $\mathrm{H}$ & -1.053889 & 2.569137 & -1.568471 & $\mathrm{C}$ & 1.138843 & -1.527378 & 0.300211 \\
\hline $\mathrm{H}$ & -1.237016 & 3.584423 & -0.132886 & $\mathrm{C}$ & 3.548406 & -2.281141 & 0.518479 \\
\hline $\mathrm{H}$ & 3.153186 & 1.835912 & -2.538365 & $\mathrm{H}$ & 2.096528 & 0.860459 & 0.161134 \\
\hline $\mathrm{H}$ & 2.979523 & 3.369704 & -1.667990 & $\mathrm{C}$ & 5.482462 & 0.373617 & 0.791475 \\
\hline $\mathrm{H}$ & 1.331622 & 0.260456 & 0.679249 & $\mathrm{C}$ & 4.120222 & 2.433265 & 0.636096 \\
\hline $\mathrm{H}$ & -0.372100 & 3.476072 & 1.930327 & 0 & 5.457535 & 1.603650 & -1.848192 \\
\hline $\mathrm{H}$ & -0.417159 & 2.464995 & 3.474684 & $\mathrm{C}$ & 0.620527 & -2.782643 & -0.417001 \\
\hline $\mathrm{H}$ & 1.232132 & 0.102429 & -2.014301 & $\mathrm{C}$ & -0.875817 & -2.982425 & -0.350774 \\
\hline $\mathrm{H}$ & 4.222998 & 0.759246 & 2.324679 & $\mathrm{C}$ & -1.791533 & -1.857147 & -0.881037 \\
\hline $\mathrm{H}$ & 5.151667 & -0.017619 & 1.053988 & 0 & -1.368142 & -3.996851 & 0.090033 \\
\hline $\mathrm{H}$ & 5.256706 & -2.542184 & 0.871013 & $\mathrm{C}$ & -3.130531 & -2.383849 & -1.413868 \\
\hline $\mathrm{H}$ & 3.882870 & -3.594433 & 1.199157 & $\mathrm{C}$ & -4.190358 & -1.589673 & -0.681165 \\
\hline $\mathrm{H}$ & 4.120183 & -3.482072 & -1.139484 & $\mathrm{C}$ & -2.200924 & -0.951454 & 0.268565 \\
\hline $\mathrm{H}$ & 3.899941 & -1.185823 & -2.040501 & $\mathrm{C}$ & -3.512780 & -0.765432 & 0.354149 \\
\hline $\mathrm{H}$ & -0.045905 & -3.611635 & -1.466908 & $\mathrm{C}$ & 0.514319 & -2.595687 & 2.500615 \\
\hline $\mathrm{H}$ & -0.165094 & -4.248949 & 0.201372 & 0 & -5.382085 & -1.622817 & -0.890337 \\
\hline $\mathrm{H}$ & -0.242966 & -2.486626 & -0.095455 & 0 & -1.084763 & -1.128368 & -1.874019 \\
\hline \multirow{2}{*}{\multicolumn{4}{|c|}{$\omega \mathrm{B} 97 \mathrm{X}$ Energy $=-1938.58740784$ a.u. }} & $\mathrm{C}$ & -4.288070 & 0.131977 & 1.260402 \\
\hline & & & & $S$ & -3.431968 & 1.653041 & 1.774785 \\
\hline \multirow{2}{*}{\multicolumn{4}{|c|}{$(3 S, 5 R, 9 S, 10 R, 13 R, 24 R)-\mathbf{1}$, Conf W }} & $\mathrm{C}$ & -3.875034 & 2.832611 & 0.466023 \\
\hline & & & & $\mathrm{C}$ & -3.192435 & 2.658244 & -0.884465 \\
\hline $\mathrm{C}$ & 4.117057 & -0.414996 & -1.965255 & $\mathrm{C}$ & -1.679780 & 2.519419 & -0.749970 \\
\hline $\mathrm{C}$ & 4.276609 & 0.967703 & -1.358838 & 0 & -3.753586 & 1.549660 & -1.540326 \\
\hline $\mathrm{C}$ & 4.202882 & 0.966820 & 0.184863 & 0 & -1.155109 & 3.532944 & -0.085479 \\
\hline $\mathrm{C}$ & 2.889671 & 0.233416 & 0.597480 & 0 & -1.029521 & 1.615564 & -1.226144 \\
\hline $\mathrm{C}$ & 2.633624 & -1.176802 & -0.024663 & $\mathrm{C}$ & 0.269942 & 3.514289 & 0.106698 \\
\hline
\end{tabular}




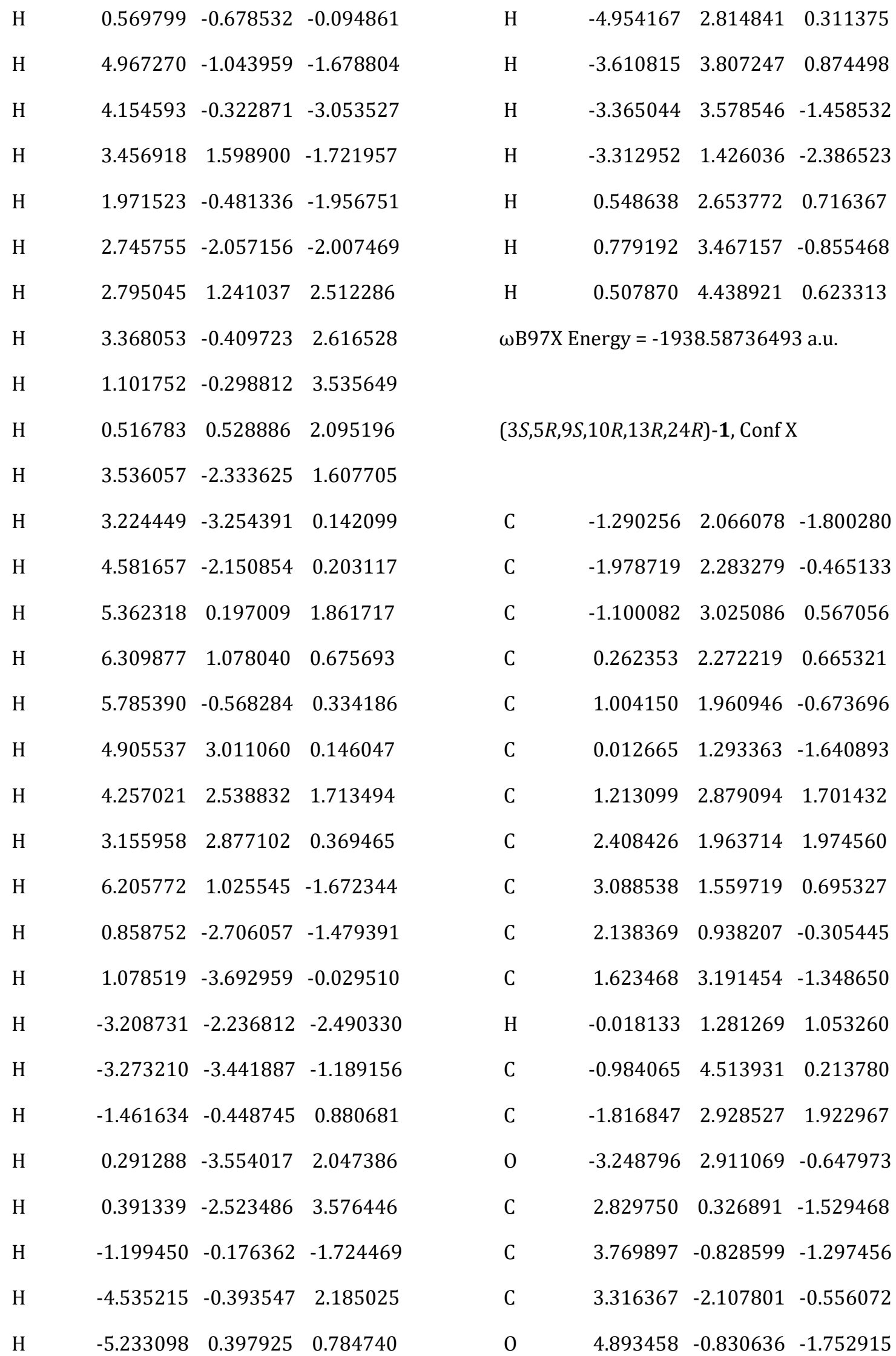




\begin{tabular}{|c|c|c|c|c|c|c|c|}
\hline $\mathrm{C}$ & 3.373090 & -1.927458 & 0.977311 & $\mathrm{H}$ & -0.240276 & 5.008564 & 0.840643 \\
\hline $\mathrm{C}$ & 2.005332 & -2.351023 & 1.474172 & $\mathrm{H}$ & -1.938097 & 5.014164 & 0.397514 \\
\hline $\mathrm{C}$ & 1.878036 & -2.498751 & -0.818943 & $\mathrm{H}$ & -2.858240 & 3.234655 & 1.809296 \\
\hline $\mathrm{C}$ & 1.155176 & -2.651311 & 0.288468 & $\mathrm{H}$ & -1.362387 & 3.579049 & 2.672250 \\
\hline $\mathrm{C}$ & 4.383592 & 1.769066 & 0.485092 & $\mathrm{H}$ & -1.802101 & 1.903646 & 2.306547 \\
\hline 0 & 1.665620 & -2.437533 & 2.630997 & $\mathrm{H}$ & -3.119178 & 3.718047 & -1.155080 \\
\hline 0 & 4.138963 & -3.172274 & -0.985567 & $\mathrm{H}$ & 2.066438 & -0.071758 & -2.205240 \\
\hline C & -0.263989 & -3.083945 & 0.415880 & $\mathrm{H}$ & 3.393367 & 1.073534 & -2.089282 \\
\hline S & -1.316208 & -1.638573 & 0.812985 & $\mathrm{H}$ & 4.145467 & -2.573258 & 1.394109 \\
\hline $\mathrm{C}$ & -2.843246 & -2.468753 & 1.337430 & $\mathrm{H}$ & 3.581086 & -0.905951 & 1.302740 \\
\hline $\mathrm{C}$ & -3.789824 & -2.834585 & 0.197115 & $\mathrm{H}$ & 1.531142 & -2.688077 & -1.829030 \\
\hline $\mathrm{C}$ & -4.276856 & -1.600478 & -0.548299 & $\mathrm{H}$ & 4.882910 & 1.494606 & -0.436899 \\
\hline 0 & -3.193482 & -3.724309 & -0.714009 & $\mathrm{H}$ & 4.996138 & 2.236332 & 1.249108 \\
\hline 0 & -4.922974 & -0.760542 & 0.238051 & $\mathrm{H}$ & 4.943983 & -2.763031 & -1.338316 \\
\hline 0 & -4.092659 & -1.442912 & -1.733020 & $\mathrm{H}$ & -0.355161 & -3.791568 & 1.241917 \\
\hline $\mathrm{C}$ & -5.470220 & 0.422078 & -0.379441 & $\mathrm{H}$ & -0.618020 & -3.553858 & -0.498244 \\
\hline $\mathrm{H}$ & 1.616476 & 0.127426 & 0.217236 & $\mathrm{H}$ & -2.579268 & -3.370737 & 1.890201 \\
\hline $\mathrm{H}$ & -1.110780 & 3.030919 & -2.287458 & $\mathrm{H}$ & -3.349913 & -1.791242 & 2.024723 \\
\hline $\mathrm{H}$ & -1.968655 & 1.510309 & -2.452962 & $\mathrm{H}$ & -4.673886 & -3.307144 & 0.641366 \\
\hline $\mathrm{H}$ & -2.215952 & 1.300099 & -0.039690 & $\mathrm{H}$ & -3.194070 & -3.286550 & -1.576355 \\
\hline $\mathrm{H}$ & -0.223136 & 0.286585 & -1.274476 & $\mathrm{H}$ & -4.669255 & 1.084755 & -0.708261 \\
\hline $\mathrm{H}$ & 0.469778 & 1.175526 & -2.626516 & $\mathrm{H}$ & -6.102362 & 0.140934 & -1.220675 \\
\hline $\mathrm{H}$ & 0.685684 & 3.052466 & 2.640509 & $\mathrm{H}$ & -6.059934 & 0.902866 & 0.395546 \\
\hline $\mathrm{H}$ & 1.577897 & 3.853205 & 1.364564 & \multicolumn{4}{|c|}{$\omega \mathrm{B} 97 \mathrm{X}$ Energy = -1938.58731634 a.u. } \\
\hline $\mathrm{H}$ & 3.119616 & 2.448944 & 2.646100 & & & & \\
\hline $\mathrm{H}$ & 2.046526 & 1.060364 & 2.483089 & \multicolumn{4}{|c|}{$(3 S, 5 R, 9 S, 10 R, 13 R, 24 R)-\mathbf{1}$, Conf $Y$} \\
\hline $\mathrm{H}$ & 0.864423 & 3.870482 & -1.731567 & & & & \\
\hline $\mathrm{H}$ & 2.267573 & 3.758117 & -0.676099 & $\mathrm{C}$ & -1.066553 & 1.899496 & -2.112949 \\
\hline $\mathrm{H}$ & 2.232336 & 2.888056 & -2.203551 & $\mathrm{C}$ & -1.953727 & 1.849801 & -0.887024 \\
\hline $\mathrm{H}$ & -0.712983 & 4.696826 & -0.826073 & $\mathrm{C}$ & -1.383750 & 2.659612 & 0.298688 \\
\hline
\end{tabular}




\begin{tabular}{|c|c|c|c|c|c|c|c|}
\hline $\mathrm{C}$ & 0.035330 & 2.084327 & 0.588419 & 0 & -4.466106 & 0.018091 & 0.187850 \\
\hline $\mathrm{C}$ & 1.020356 & 1.959903 & -0.621768 & $\mathrm{C}$ & -4.797243 & -1.308717 & 2.472831 \\
\hline $\mathrm{C}$ & 0.301852 & 1.299811 & -1.810934 & $\mathrm{H}$ & 1.631764 & 0.140869 & 0.288713 \\
\hline $\mathrm{C}$ & 0.726417 & 2.726399 & 1.795307 & $\mathrm{H}$ & -0.982163 & 2.928457 & -2.475212 \\
\hline $\mathrm{C}$ & 1.968738 & 1.937240 & 2.217900 & $\mathrm{H}$ & -1.545398 & 1.325335 & -2.910593 \\
\hline C & 2.879184 & 1.659425 & 1.052454 & $\mathrm{H}$ & -2.002345 & 0.804391 & -0.556255 \\
\hline $\mathrm{C}$ & 2.163551 & 1.010982 & -0.112742 & $\mathrm{H}$ & 0.165959 & 0.232806 & -1.605642 \\
\hline $\mathrm{C}$ & 1.621205 & 3.292388 & -1.089826 & $\mathrm{H}$ & 0.927096 & 1.373231 & -2.705158 \\
\hline $\mathrm{H}$ & -0.168421 & 1.041846 & 0.876154 & $\mathrm{H}$ & 0.041814 & 2.768290 & 2.643251 \\
\hline $\mathrm{C}$ & -1.418053 & 4.165104 & 0.021334 & $\mathrm{H}$ & 1.007339 & 3.759383 & 1.573677 \\
\hline C & -2.281339 & 2.392476 & 1.516728 & $\mathrm{H}$ & 2.511087 & 2.464280 & 3.005631 \\
\hline 0 & -3.267975 & 2.258232 & -1.268003 & $\mathrm{H}$ & 1.640184 & 0.977607 & 2.639280 \\
\hline $\mathrm{C}$ & 3.091415 & 0.497864 & -1.218532 & $\mathrm{H}$ & 2.013805 & 3.895582 & -0.271620 \\
\hline $\mathrm{C}$ & 4.064386 & -0.592699 & -0.844503 & $\mathrm{H}$ & 2.447222 & 3.107928 & -1.781420 \\
\hline $\mathrm{C}$ & 3.593502 & -1.939655 & -0.236142 & $\mathrm{H}$ & 0.890517 & 3.893862 & -1.628817 \\
\hline 0 & 5.249470 & -0.487443 & -1.076606 & $\mathrm{H}$ & -2.453600 & 4.494114 & -0.085058 \\
\hline $\mathrm{C}$ & 3.242476 & -1.807900 & 1.263368 & $\mathrm{H}$ & -0.889701 & 4.450860 & -0.886868 \\
\hline $\mathrm{C}$ & 1.803630 & -2.271086 & 1.377675 & $\mathrm{H}$ & -0.976779 & 4.719966 & 0.852454 \\
\hline $\mathrm{C}$ & 2.321284 & -2.481132 & -0.853228 & $\mathrm{H}$ & -3.318547 & 2.651680 & 1.292624 \\
\hline $\mathrm{C}$ & 1.345825 & -2.696176 & 0.027309 & $\mathrm{H}$ & -1.987258 & 2.994057 & 2.377828 \\
\hline $\mathrm{C}$ & 4.167194 & 1.984967 & 1.066436 & $\mathrm{H}$ & -2.252188 & 1.337619 & 1.807444 \\
\hline 0 & 1.140815 & -2.291879 & 2.389050 & $\mathrm{H}$ & -3.887114 & 1.841224 & -0.658529 \\
\hline 0 & 4.617324 & -2.886905 & -0.448561 & $\mathrm{H}$ & 2.481394 & 0.068099 & -2.019458 \\
\hline $\mathrm{C}$ & -0.011648 & -3.269495 & -0.210234 & $\mathrm{H}$ & 3.681719 & 1.301519 & -1.659918 \\
\hline S & -1.206770 & -1.908619 & -0.438158 & $\mathrm{H}$ & 3.885547 & -2.478847 & 1.833557 \\
\hline $\mathrm{C}$ & -2.692108 & -2.869455 & -0.862173 & $\mathrm{H}$ & 3.358420 & -0.805904 & 1.679113 \\
\hline $\mathrm{C}$ & -3.891013 & -1.950740 & -1.066830 & $\mathrm{H}$ & 2.279487 & -2.712741 & -1.912097 \\
\hline $\mathrm{C}$ & -4.274069 & -1.177893 & 0.189906 & $\mathrm{H}$ & 4.832250 & 1.793337 & 0.232333 \\
\hline 0 & -3.663309 & -1.059611 & -2.131591 & $\mathrm{H}$ & 4.603202 & 2.466741 & 1.935395 \\
\hline 0 & -4.410318 & -1.954394 & 1.244647 & $\mathrm{H}$ & 5.413947 & -2.375614 & -0.661474 \\
\hline
\end{tabular}




\begin{tabular}{|c|c|c|c|c|c|c|c|}
\hline $\mathrm{H}$ & -0.313047 & -3.873555 & 0.646482 & $\mathrm{C}$ & -2.802111 & 2.857117 & 0.731740 \\
\hline $\mathrm{H}$ & -0.010991 & -3.887611 & -1.107779 & $\mathrm{C}$ & -2.586067 & 2.280578 & -1.576468 \\
\hline $\mathrm{H}$ & -2.514736 & -3.415239 & -1.789377 & $\mathrm{C}$ & -3.274770 & 1.274578 & -1.037831 \\
\hline $\mathrm{H}$ & -2.914236 & -3.580265 & -0.066571 & $\mathrm{C}$ & -3.376389 & 1.493393 & 0.425239 \\
\hline $\mathrm{H}$ & -4.751208 & -2.592503 & -1.296641 & 0 & -3.842376 & 0.720341 & 1.236631 \\
\hline $\mathrm{H}$ & -3.930793 & -0.172293 & -1.855716 & $\mathrm{C}$ & -3.808096 & 0.053309 & -1.700991 \\
\hline $\mathrm{H}$ & -4.050116 & -0.566906 & 2.752418 & S & -2.558419 & -1.285595 & -1.549914 \\
\hline $\mathrm{H}$ & -5.768479 & -0.831573 & 2.351357 & $\mathrm{C}$ & -3.574393 & -2.738094 & -1.146026 \\
\hline $\mathrm{H}$ & -4.847582 & -2.099921 & 3.213921 & $\mathrm{C}$ & -3.905318 & -2.898306 & 0.339523 \\
\hline \multicolumn{4}{|c|}{$\omega B 97 X$ Energy = -1938.58731529 a.u. } & $\mathrm{C}$ & -2.632423 & -2.968654 & 1.178233 \\
\hline & & & & 0 & -4.780580 & -1.921673 & 0.827825 \\
\hline & & & & 0 & -1.853735 & -3.974182 & 0.786604 \\
\hline & & & & 0 & -2.363129 & -2.212951 & 2.076999 \\
\hline \multicolumn{4}{|c|}{$(3 S, 5 R, 9 S, 10 R, 13 S, 24 R)-\mathbf{1}$, Conf A } & $\mathrm{C}$ & -0.618399 & -4.141114 & 1.499882 \\
\hline & & & & $\mathrm{C}$ & 4.520154 & -2.021405 & 0.684341 \\
\hline $\mathrm{C}$ & 2.734954 & -1.427094 & -1.882397 & $\mathrm{C}$ & 6.039132 & -0.302186 & -0.240776 \\
\hline $\mathrm{C}$ & 4.217870 & -1.167760 & -1.684770 & $\mathrm{C}$ & 1.459603 & -0.795438 & 0.927326 \\
\hline $\mathrm{C}$ & 4.582087 & -0.790355 & -0.230787 & 0 & 4.994011 & -2.260618 & -2.176571 \\
\hline $\mathrm{C}$ & 3.658621 & 0.389976 & 0.201607 & $\mathrm{H}$ & 3.934191 & 1.200627 & -0.489946 \\
\hline $\mathrm{C}$ & 2.121959 & 0.215000 & -0.018690 & 0 & -2.319368 & 4.588283 & -0.939315 \\
\hline $\mathrm{C}$ & 1.892119 & -0.225456 & -1.475095 & $\mathrm{H}$ & 2.435184 & -2.316565 & -1.316988 \\
\hline $\mathrm{C}$ & 3.969047 & 0.915240 & 1.606235 & $\mathrm{H}$ & 2.560286 & -1.658420 & -2.936034 \\
\hline $\mathrm{C}$ & 3.263155 & 2.245169 & 1.871565 & $\mathrm{H}$ & 4.505581 & -0.322283 & -2.320238 \\
\hline $\mathrm{C}$ & 1.789014 & 2.132236 & 1.600326 & $\mathrm{H}$ & 2.127937 & 0.613411 & -2.141758 \\
\hline $\mathrm{C}$ & 1.480416 & 1.629377 & 0.204734 & $\mathrm{H}$ & 0.839000 & -0.472293 & -1.637204 \\
\hline $\mathrm{C}$ & 0.886255 & 2.412357 & 2.532636 & $\mathrm{H}$ & 5.043843 & 1.058799 & 1.726336 \\
\hline $\mathrm{C}$ & 0.002142 & 1.693326 & -0.161198 & $\mathrm{H}$ & 3.664558 & 0.189167 & 2.365235 \\
\hline $\mathrm{C}$ & -0.527888 & 3.082956 & -0.394231 & $\mathrm{H}$ & 3.436813 & 2.574971 & 2.897973 \\
\hline $\mathrm{C}$ & -2.059538 & 3.264907 & -0.557246 & $\mathrm{H}$ & 3.687755 & 3.007033 & 1.206501 \\
\hline 0 & 0.169696 & 4.063203 & -0.513820 & $\mathrm{H}$ & 2.001024 & 2.304160 & -0.485919 \\
\hline
\end{tabular}




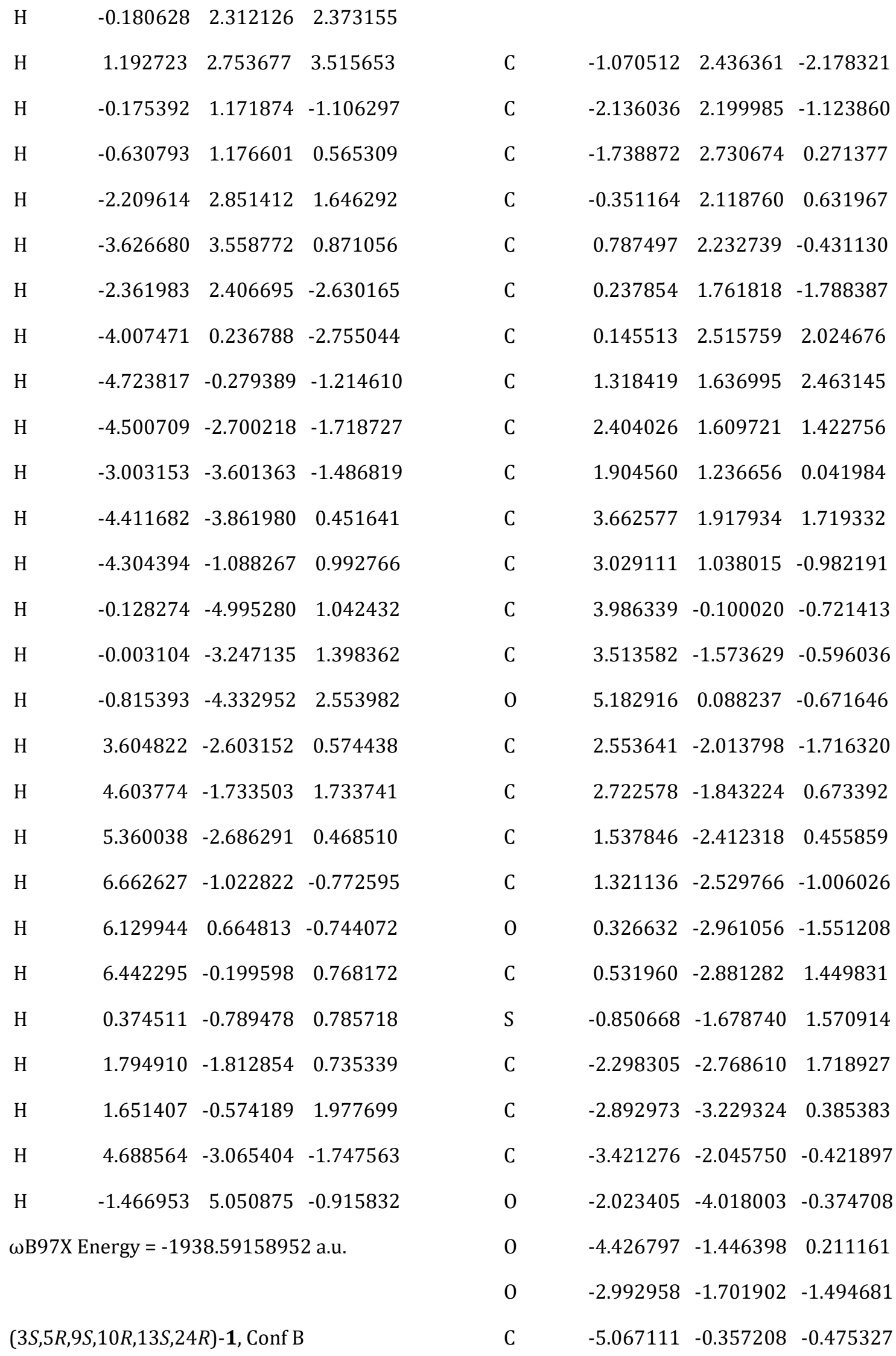




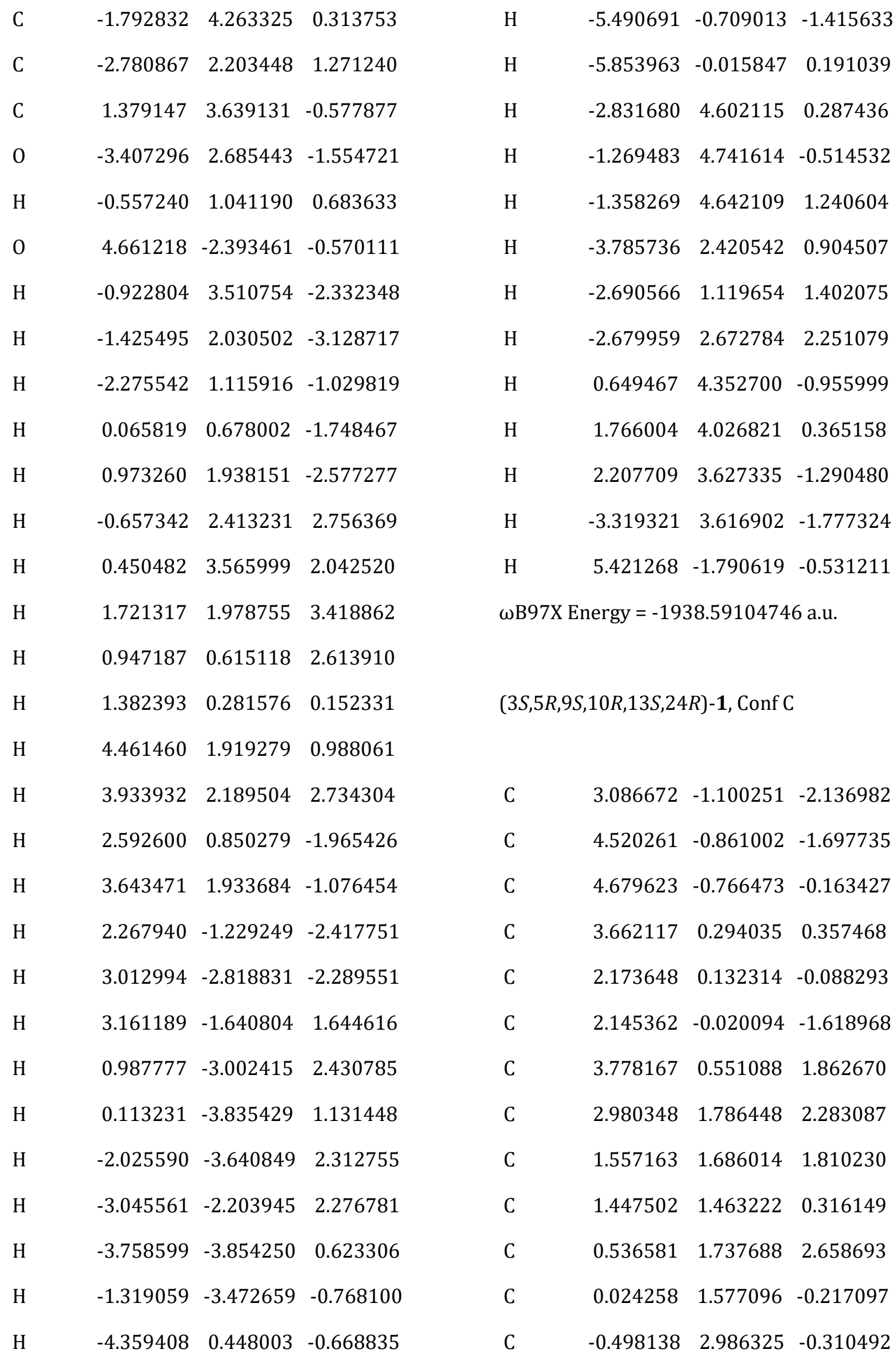




\begin{tabular}{|c|c|c|c|c|c|c|c|}
\hline $\mathrm{C}$ & -2.011407 & 3.193781 & -0.581421 & $\mathrm{H}$ & 3.442303 & 2.673552 & 1.833179 \\
\hline 0 & 0.196829 & 3.972433 & -0.230925 & $\mathrm{H}$ & 2.021636 & 2.269003 & -0.156882 \\
\hline $\mathrm{C}$ & -2.830105 & 2.751334 & 0.651038 & $\mathrm{H}$ & -0.496888 & 1.629300 & 2.351591 \\
\hline $\mathrm{C}$ & -2.487888 & 2.252435 & -1.661270 & $\mathrm{H}$ & 0.707023 & 1.883075 & 3.720066 \\
\hline $\mathrm{C}$ & -3.208945 & 1.232420 & -1.195338 & $\mathrm{H}$ & -0.029287 & 1.203352 & -1.244384 \\
\hline $\mathrm{C}$ & -3.399461 & 1.407449 & 0.264346 & $\mathrm{H}$ & -0.682820 & 0.955056 & 0.337881 \\
\hline 0 & -3.922413 & 0.613939 & 1.019942 & $\mathrm{H}$ & -2.286728 & 2.706094 & 1.594924 \\
\hline $\mathrm{C}$ & -3.682931 & 0.020569 & -1.918297 & $\mathrm{H}$ & -3.652188 & 3.459455 & 0.770524 \\
\hline S & -2.423628 & -1.299425 & -1.692029 & $\mathrm{H}$ & -2.200498 & 2.407979 & -2.695411 \\
\hline $\mathrm{C}$ & -3.431852 & -2.780355 & -1.384442 & $\mathrm{H}$ & -3.807223 & 0.219424 & -2.980990 \\
\hline $\mathrm{C}$ & -3.874228 & -2.961483 & 0.068265 & $\mathrm{H}$ & -4.626925 & -0.332612 & -1.505909 \\
\hline $\mathrm{C}$ & -2.642605 & -3.063806 & 0.967171 & $\mathrm{H}$ & -4.307855 & -2.765337 & -2.032215 \\
\hline 0 & -4.817611 & -2.015509 & 0.491071 & $\mathrm{H}$ & -2.807788 & -3.623986 & -1.679043 \\
\hline 0 & -2.667222 & -2.213735 & 1.983309 & $\mathrm{H}$ & -4.363494 & -3.938656 & 0.132082 \\
\hline 0 & -1.746079 & -3.846386 & 0.758235 & $\mathrm{H}$ & -4.386610 & -1.165243 & 0.689482 \\
\hline $\mathrm{C}$ & -1.534281 & -2.236442 & 2.865198 & $\mathrm{H}$ & -1.734791 & -1.480550 & 3.618798 \\
\hline $\mathrm{C}$ & 4.544780 & -2.151003 & 0.485540 & $\mathrm{H}$ & -0.625152 & -1.994577 & 2.314462 \\
\hline $\mathrm{C}$ & 6.106120 & -0.267733 & 0.115486 & $\mathrm{H}$ & -1.434722 & -3.218646 & 3.325617 \\
\hline $\mathrm{C}$ & 1.444559 & -1.055055 & 0.555002 & $\mathrm{H}$ & 5.431610 & -2.751042 & 0.267404 \\
\hline 0 & 5.395201 & -1.828138 & -2.278808 & $\mathrm{H}$ & 3.675334 & -2.711896 & 0.142628 \\
\hline $\mathrm{H}$ & 3.984246 & 1.225820 & -0.131837 & $\mathrm{H}$ & 4.475781 & -2.068016 & 1.571541 \\
\hline 0 & -2.228474 & 4.532442 & -0.935085 & $\mathrm{H}$ & 6.818680 & -0.861229 & -0.459707 \\
\hline $\mathrm{H}$ & 2.757493 & -2.089873 & -1.800957 & $\mathrm{H}$ & 6.224147 & 0.781323 & -0.171411 \\
\hline $\mathrm{H}$ & 3.056696 & -1.121216 & -3.229150 & $\mathrm{H}$ & 6.372167 & -0.364655 & 1.169523 \\
\hline $\mathrm{H}$ & 4.853999 & 0.094843 & -2.117590 & $\mathrm{H}$ & 1.542713 & -1.061774 & 1.641490 \\
\hline $\mathrm{H}$ & 2.420632 & 0.938293 & -2.076610 & $\mathrm{H}$ & 0.376685 & -1.011893 & 0.319418 \\
\hline $\mathrm{H}$ & 1.133262 & -0.254313 & -1.960369 & $\mathrm{H}$ & 1.805458 & -2.011697 & 0.182808 \\
\hline $\mathrm{H}$ & 4.823317 & 0.698667 & 2.138147 & $\mathrm{H}$ & 5.070026 & -2.704058 & -2.050561 \\
\hline $\mathrm{H}$ & 3.424984 & -0.314497 & 2.430386 & $\mathrm{H}$ & -1.405329 & 5.004523 & -0.734780 \\
\hline $\mathrm{H}$ & 3.014383 & 1.915311 & 3.366899 & & eergy $=-1$ & 38.5905715 & 1 a.u. \\
\hline
\end{tabular}




\begin{tabular}{|c|c|c|c|c|c|c|c|}
\hline \multirow{2}{*}{\multicolumn{3}{|c|}{$(3 S, 5 R, 9 S, 10 R, 13 S, 24 R)-\mathbf{1}$, Conf D }} & & \multirow[b]{2}{*}{$\mathrm{C}$} & \multirow{2}{*}{$\begin{array}{l}3.246303 \\
1.463764\end{array}$} & \multirow{2}{*}{$\begin{array}{l}-1.925056 \\
-3.855236\end{array}$} & \multirow{2}{*}{$\begin{array}{l}-1.470551 \\
-1.058545\end{array}$} \\
\hline & & & & & & & \\
\hline $\mathrm{C}$ & -2.832467 & 0.640577 & 2.114923 & $\mathrm{C}$ & -5.898353 & -0.656816 & 0.156423 \\
\hline $\mathrm{C}$ & -4.278141 & 0.392499 & 1.717222 & $\mathrm{C}$ & -1.206517 & -1.255194 & 0.214129 \\
\hline $\mathrm{C}$ & -4.439169 & -0.700881 & 0.634425 & 0 & -5.084045 & 0.143342 & 2.869001 \\
\hline $\mathrm{C}$ & -3.504632 & -0.334835 & -0.559692 & $\mathrm{H}$ & -3.904937 & 0.622691 & -0.925753 \\
\hline $\mathrm{C}$ & -2.014994 & -0.020468 & -0.213278 & 0 & 1.065260 & 4.677482 & -0.138671 \\
\hline $\mathrm{C}$ & -1.987018 & 1.028030 & 0.908734 & $\mathrm{H}$ & -2.426047 & -0.248326 & 2.609103 \\
\hline $\mathrm{C}$ & -3.613250 & -1.316276 & -1.732102 & $\mathrm{H}$ & -2.810173 & 1.443316 & 2.855898 \\
\hline $\mathrm{C}$ & -2.845626 & -0.830600 & -2.965071 & $\mathrm{H}$ & -4.681684 & 1.321777 & 1.299036 \\
\hline $\mathrm{C}$ & -1.434936 & -0.444083 & -2.613656 & $\mathrm{H}$ & -2.360345 & 1.979599 & 0.516013 \\
\hline $\mathrm{C}$ & -1.384052 & 0.592587 & -1.511961 & $\mathrm{H}$ & -0.954488 & 1.198415 & 1.234003 \\
\hline $\mathrm{C}$ & -0.379919 & -1.014436 & -3.185311 & $\mathrm{H}$ & -4.660226 & -1.456521 & -2.005539 \\
\hline $\mathrm{C}$ & -0.003821 & 1.227575 & -1.324807 & $\mathrm{H}$ & -3.234514 & -2.300773 & -1.444577 \\
\hline $\mathrm{C}$ & -0.017342 & 2.681074 & -0.922165 & $\mathrm{H}$ & -2.849782 & -1.598724 & -3.741253 \\
\hline $\mathrm{C}$ & 1.258869 & 3.297105 & -0.292288 & $\mathrm{H}$ & -3.356443 & 0.049601 & -3.373824 \\
\hline 0 & -0.951030 & 3.423651 & -1.123928 & $\mathrm{H}$ & -2.053879 & 1.403772 & -1.819776 \\
\hline $\mathrm{C}$ & 2.509112 & 2.966636 & -1.129853 & $\mathrm{H}$ & 0.642885 & -0.769251 & -2.919051 \\
\hline $\mathrm{C}$ & 1.557602 & 2.635996 & 1.036467 & $\mathrm{H}$ & -0.510262 & -1.771222 & -3.952118 \\
\hline $\mathrm{C}$ & 2.611377 & 1.820445 & 1.000243 & $\mathrm{H}$ & 0.637350 & 0.656750 & -0.649876 \\
\hline $\mathrm{C}$ & 3.204593 & 1.870407 & -0.358869 & $\mathrm{H}$ & 0.515062 & 1.229304 & -2.289917 \\
\hline 0 & 4.096699 & 1.163432 & -0.779266 & $\mathrm{H}$ & 2.332283 & 2.685475 & -2.167123 \\
\hline $\mathrm{C}$ & 3.121864 & 0.909290 & 2.062673 & $\mathrm{H}$ & 3.149721 & 3.850822 & -1.122795 \\
\hline$S$ & 2.234335 & -0.692849 & 1.933463 & $\mathrm{H}$ & 0.930144 & 2.830161 & 1.899778 \\
\hline $\mathrm{C}$ & 3.558119 & -1.934241 & 2.021775 & $\mathrm{H}$ & 2.949875 & 1.329296 & 3.051888 \\
\hline $\mathrm{C}$ & 4.278319 & -2.211813 & 0.700429 & $\mathrm{H}$ & 4.187713 & 0.726310 & 1.934075 \\
\hline $\mathrm{C}$ & 3.281821 & -2.509522 & -0.417509 & $\mathrm{H}$ & 4.290090 & -1.640617 & 2.773712 \\
\hline 0 & 5.169972 & -1.198947 & 0.327351 & $\mathrm{H}$ & 3.067852 & -2.844421 & 2.366698 \\
\hline 0 & 2.456013 & -3.497193 & -0.082573 & $\mathrm{H}$ & 4.873107 & -3.119514 & 0.844866 \\
\hline
\end{tabular}




\begin{tabular}{|c|c|c|c|c|c|c|c|}
\hline $\mathrm{H}$ & 4.693637 & -0.464047 & -0.098529 & C & -3.156652 & 0.098699 & 0.665467 \\
\hline $\mathrm{H}$ & 0.839146 & -2.992848 & -1.295555 & $\mathrm{C}$ & -3.844408 & -0.880214 & -0.250922 \\
\hline $\mathrm{H}$ & 1.944347 & -4.220188 & -1.965735 & $\mathrm{C}$ & -3.228833 & -2.283464 & -0.459345 \\
\hline $\mathrm{H}$ & 0.871459 & -4.641389 & -0.599685 & 0 & -4.887384 & -0.643577 & -0.818473 \\
\hline $\mathrm{H}$ & -4.993478 & -2.349261 & 1.925458 & $\mathrm{C}$ & -3.242668 & -3.052143 & 0.880793 \\
\hline $\mathrm{H}$ & -3.245735 & -2.179968 & 1.760639 & $\mathrm{C}$ & -1.761879 & -2.242581 & -0.827476 \\
\hline $\mathrm{H}$ & -4.200458 & -2.857401 & 0.447354 & $\mathrm{C}$ & -0.960531 & -2.764761 & 0.098726 \\
\hline $\mathrm{H}$ & -6.568955 & -0.688948 & 1.016745 & $\mathrm{C}$ & -1.784611 & -3.229630 & 1.247374 \\
\hline $\mathrm{H}$ & -6.103543 & 0.261255 & -0.402003 & 0 & -1.358229 & -3.665255 & 2.291978 \\
\hline $\mathrm{H}$ & -6.142298 & -1.506363 & -0.483856 & $\mathrm{C}$ & 0.520314 & -2.925902 & 0.147591 \\
\hline $\mathrm{H}$ & -1.387677 & -1.520698 & 1.254486 & S & 1.365339 & -2.077583 & -1.223241 \\
\hline $\mathrm{H}$ & -1.428224 & -2.131220 & -0.395530 & $\mathrm{C}$ & 2.965964 & -2.933517 & -1.228689 \\
\hline $\mathrm{H}$ & -0.134222 & -1.066063 & 0.127867 & $\mathrm{C}$ & 3.948026 & -2.494136 & -0.152544 \\
\hline $\mathrm{H}$ & -4.694673 & -0.588594 & 3.356282 & $\mathrm{C}$ & 4.245450 & -1.003355 & -0.191949 \\
\hline $\mathrm{H}$ & 0.156494 & 4.858827 & -0.425957 & 0 & 3.475965 & -2.845256 & 1.124480 \\
\hline \multirow{2}{*}{\multicolumn{4}{|c|}{$\omega B 97 X$ Energy = -1938.59044931 a.u. }} & 0 & 4.558807 & -0.569161 & -1.393420 \\
\hline & & & & 0 & 4.227586 & -0.321681 & 0.811321 \\
\hline \multirow{2}{*}{\multicolumn{4}{|c|}{$(3 S, 5 R, 9 S, 10 R, 13 S, 24 R)-\mathbf{1}$, Conf $\mathrm{E}$}} & $\mathrm{C}$ & 4.923779 & 0.820464 & -1.513078 \\
\hline & & & & $\mathrm{C}$ & 1.053383 & 4.251507 & 1.169170 \\
\hline $\mathrm{C}$ & 1.078640 & 1.156905 & 1.815102 & $\mathrm{C}$ & 1.892800 & 3.586176 & -1.063275 \\
\hline $\mathrm{C}$ & 1.857011 & 1.887595 & 0.735922 & $\mathrm{C}$ & -1.743392 & 2.587105 & 1.858778 \\
\hline $\mathrm{C}$ & 1.101569 & 3.098014 & 0.160752 & 0 & 3.132984 & 2.301487 & 1.216839 \\
\hline $\mathrm{C}$ & -0.297178 & 2.595561 & -0.312616 & $\mathrm{H}$ & -0.056762 & 1.870068 & -1.104950 \\
\hline $\mathrm{C}$ & -1.148204 & 1.767696 & 0.707019 & 0 & -3.949664 & -2.941741 & -1.470556 \\
\hline $\mathrm{C}$ & -0.256447 & 0.653462 & 1.282976 & $\mathrm{H}$ & 0.943447 & 1.813295 & 2.679633 \\
\hline $\mathrm{C}$ & -1.131660 & 3.687922 & -0.984784 & $\mathrm{H}$ & 1.674437 & 0.305434 & 2.159479 \\
\hline $\mathrm{C}$ & -2.340668 & 3.092021 & -1.703262 & $\mathrm{H}$ & 2.002920 & 1.182469 & -0.098328 \\
\hline $\mathrm{C}$ & -3.147006 & 2.221562 & -0.778656 & $\mathrm{H}$ & -0.055631 & -0.080906 & 0.494417 \\
\hline $\mathrm{C}$ & -2.321068 & 1.128967 & -0.125484 & $\mathrm{H}$ & -0.773820 & 0.124992 & 2.088113 \\
\hline $\mathrm{C}$ & -4.432846 & 2.463137 & -0.553688 & $\mathrm{H}$ & -0.526278 & 4.233892 & -1.709484 \\
\hline
\end{tabular}




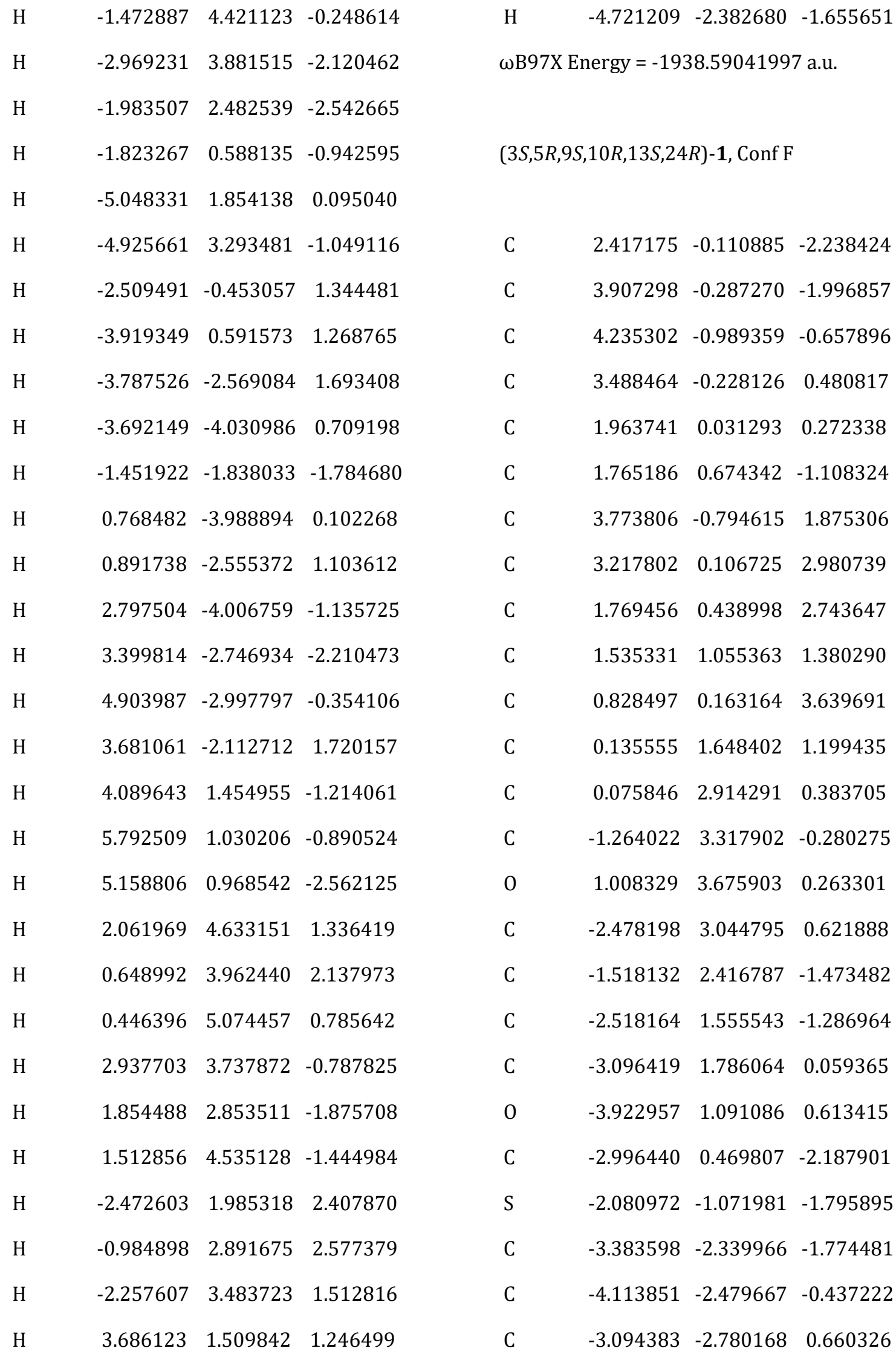




\begin{tabular}{|c|c|c|c|c|c|c|c|}
\hline 0 & -4.975061 & -1.412209 & -0.151761 & $\mathrm{H}$ & -2.878816 & -3.277396 & -2.007483 \\
\hline 0 & -3.143464 & -1.923068 & 1.670190 & $\mathrm{H}$ & -4.741707 & -3.373273 & -0.507937 \\
\hline 0 & -2.321172 & -3.706146 & 0.593823 & $\mathrm{H}$ & -4.477311 & -0.628631 & 0.140912 \\
\hline C & -2.186535 & -2.122077 & 2.722713 & $\mathrm{H}$ & -2.307419 & -3.112038 & 3.160630 \\
\hline $\mathrm{C}$ & 3.915024 & -2.488181 & -0.736843 & $\mathrm{H}$ & -2.397201 & -1.351848 & 3.459078 \\
\hline $\mathrm{C}$ & 5.749323 & -0.855300 & -0.433321 & $\mathrm{H}$ & -1.173292 & -2.011397 & 2.334077 \\
\hline C & 1.100758 & -1.233899 & 0.383275 & $\mathrm{H}$ & 4.624592 & -2.984800 & -1.403305 \\
\hline 0 & 4.530071 & -0.922556 & -3.113250 & $\mathrm{H}$ & 2.911367 & -2.701567 & -1.103976 \\
\hline $\mathrm{H}$ & 3.936969 & 0.776608 & 0.463627 & $\mathrm{H}$ & 4.015636 & -2.962232 & 0.240915 \\
\hline 0 & -1.189247 & 4.665063 & -0.667825 & $\mathrm{H}$ & 6.283695 & -1.177804 & -1.328504 \\
\hline $\mathrm{H}$ & 1.940542 & -1.089728 & -2.359443 & $\mathrm{H}$ & 6.027705 & 0.181706 & -0.224056 \\
\hline $\mathrm{H}$ & 2.279867 & 0.418214 & -3.184658 & $\mathrm{H}$ & 6.093095 & -1.474526 & 0.397074 \\
\hline $\mathrm{H}$ & 4.366318 & 0.707279 & -1.959300 & $\mathrm{H}$ & 1.356735 & -1.838825 & 1.253378 \\
\hline $\mathrm{H}$ & 2.194121 & 1.682532 & -1.095199 & $\mathrm{H}$ & 0.043072 & -0.974492 & 0.467856 \\
\hline $\mathrm{H}$ & 0.696102 & 0.783718 & -1.318634 & $\mathrm{H}$ & 1.185392 & -1.866507 & -0.498269 \\
\hline $\mathrm{H}$ & 4.849319 & -0.904256 & 2.022939 & $\mathrm{H}$ & 4.073110 & -1.751583 & -3.282984 \\
\hline $\mathrm{H}$ & 3.343571 & -1.794121 & 1.980571 & $\mathrm{H}$ & -0.253871 & 4.914222 & -0.612147 \\
\hline $\mathrm{H}$ & 3.345377 & -0.365543 & 3.957046 & \multicolumn{4}{|c|}{$\omega B 97 X$ Energy = -1938.58967903 a.u. } \\
\hline $\mathrm{H}$ & 3.791448 & 1.041472 & 2.992532 & & & & \\
\hline $\mathrm{H}$ & 2.234663 & 1.895807 & 1.299913 & \multirow{2}{*}{\multicolumn{4}{|c|}{$(3 S, 5 R, 9 S, 10 R, 13 S, 24 R)-\mathbf{1}$, Conf G }} \\
\hline $\mathrm{H}$ & -0.222675 & 0.374665 & 3.478237 & & & & \\
\hline $\mathrm{H}$ & 1.086752 & -0.299566 & 4.586511 & $\mathrm{C}$ & -1.120539 & 2.145989 & -2.007392 \\
\hline $\mathrm{H}$ & -0.589727 & 0.922108 & 0.825864 & $\mathrm{C}$ & -1.977572 & 2.336184 & -0.769097 \\
\hline $\mathrm{H}$ & -0.244547 & 1.964798 & 2.177257 & $\mathrm{C}$ & -1.243911 & 3.084258 & 0.366297 \\
\hline $\mathrm{H}$ & -2.273946 & 2.955245 & 1.687602 & $\mathrm{C}$ & 0.096319 & 2.334306 & 0.633826 \\
\hline $\mathrm{H}$ & -3.185785 & 3.864190 & 0.479493 & $\mathrm{C}$ & 1.009597 & 2.026499 & -0.596796 \\
\hline $\mathrm{H}$ & -0.909450 & 2.505748 & -2.366989 & $\mathrm{C}$ & 0.148131 & 1.367864 & -1.688734 \\
\hline $\mathrm{H}$ & -2.827256 & 0.727581 & -3.231678 & $\mathrm{C}$ & 0.901456 & 2.921683 & 1.796396 \\
\hline $\mathrm{H}$ & -4.059085 & 0.286768 & -2.034679 & $\mathrm{C}$ & 2.014170 & 1.966860 & 2.230671 \\
\hline $\mathrm{H}$ & -4.106722 & -2.139198 & -2.564357 & $\mathrm{C}$ & 2.871972 & 1.565742 & 1.062131 \\
\hline
\end{tabular}




\begin{tabular}{|c|c|c|c|c|c|c|c|}
\hline $\mathrm{C}$ & 2.073548 & 0.989581 & -0.088624 & $\mathrm{H}$ & 0.724595 & 1.250011 & -2.609605 \\
\hline $\mathrm{C}$ & 4.188434 & 1.746790 & 1.065882 & $\mathrm{H}$ & 0.248472 & 3.109014 & 2.650171 \\
\hline $\mathrm{C}$ & 2.939457 & 0.400008 & -1.213148 & $\mathrm{H}$ & 1.338006 & 3.885604 & 1.520048 \\
\hline $\mathrm{C}$ & 3.772287 & -0.806557 & -0.853921 & $\mathrm{H}$ & 2.627709 & 2.417542 & 3.013558 \\
\hline $\mathrm{C}$ & 3.131222 & -2.170425 & -0.478808 & $\mathrm{H}$ & 1.552730 & 1.065959 & 2.655631 \\
\hline 0 & 4.982511 & -0.778808 & -0.914860 & $\mathrm{H}$ & 1.477211 & 0.174641 & 0.332168 \\
\hline $\mathrm{C}$ & 2.090449 & -2.656119 & -1.507584 & $\mathrm{H}$ & 4.826449 & 1.473564 & 0.234497 \\
\hline $\mathrm{C}$ & 2.347715 & -2.126352 & 0.820067 & $\mathrm{H}$ & 4.677132 & 2.184481 & 1.930293 \\
\hline $\mathrm{C}$ & 1.085334 & -2.534101 & 0.700578 & $\mathrm{H}$ & 2.293590 & 0.099780 & -2.039760 \\
\hline $\mathrm{C}$ & 0.809285 & -2.837053 & -0.723318 & $\mathrm{H}$ & 3.636129 & 1.141405 & -1.604750 \\
\hline 0 & -0.260368 & -3.171039 & -1.189883 & $\mathrm{H}$ & 1.917003 & -1.986096 & -2.349928 \\
\hline $\mathrm{C}$ & 0.050112 & -2.679961 & 1.761099 & $\mathrm{H}$ & 2.410826 & -3.617461 & -1.909443 \\
\hline$S$ & -1.129121 & -1.275298 & 1.689788 & $\mathrm{H}$ & 2.838933 & -1.846589 & 1.746089 \\
\hline $\mathrm{C}$ & -2.712369 & -2.098369 & 2.038589 & $\mathrm{H}$ & 0.510135 & -2.712949 & 2.747000 \\
\hline $\mathrm{C}$ & -3.415300 & -2.653485 & 0.799953 & $\mathrm{H}$ & -0.515106 & -3.597909 & 1.602291 \\
\hline $\mathrm{C}$ & -3.837154 & -1.500878 & -0.111526 & $\mathrm{H}$ & -2.546897 & -2.899374 & 2.758426 \\
\hline 0 & -2.708658 & -3.676292 & 0.155885 & $\mathrm{H}$ & -3.348359 & -1.348367 & 2.507893 \\
\hline 0 & -3.445479 & -1.648878 & -1.368880 & $\mathrm{H}$ & -4.355194 & -3.098308 & 1.142360 \\
\hline 0 & -4.480001 & -0.560170 & 0.291751 & $\mathrm{H}$ & -1.925047 & -3.324456 & -0.302130 \\
\hline $\mathrm{C}$ & -3.874344 & -0.634685 & -2.293630 & $\mathrm{H}$ & -3.359553 & -0.851023 & -3.225196 \\
\hline $\mathrm{C}$ & -1.087181 & 4.573570 & 0.034869 & $\mathrm{H}$ & -3.612471 & 0.357520 & -1.926620 \\
\hline $\mathrm{C}$ & -2.126210 & 2.980534 & 1.620164 & $\mathrm{H}$ & -4.953397 & -0.697128 & -2.432852 \\
\hline $\mathrm{C}$ & 1.722121 & 3.253760 & -1.177402 & $\mathrm{H}$ & -2.058669 & 5.071554 & 0.086511 \\
\hline 0 & -3.231195 & 2.930611 & -1.103860 & $\mathrm{H}$ & -0.672773 & 4.760092 & -0.955854 \\
\hline $\mathrm{H}$ & -0.232794 & 1.342429 & 0.972513 & $\mathrm{H}$ & -0.437935 & 5.065845 & 0.761341 \\
\hline 0 & 4.170829 & -3.109864 & -0.327474 & $\mathrm{H}$ & -3.148547 & 3.273425 & 1.375164 \\
\hline $\mathrm{H}$ & -0.878919 & 3.119536 & -2.447357 & $\mathrm{H}$ & -2.147665 & 1.955658 & 2.004603 \\
\hline $\mathrm{H}$ & -1.702926 & 1.606369 & -2.758881 & $\mathrm{H}$ & -1.777481 & 3.637034 & 2.419108 \\
\hline $\mathrm{H}$ & -2.241446 & 1.345715 & -0.376812 & $\mathrm{H}$ & 2.279042 & 3.807395 & -0.420873 \\
\hline $\mathrm{H}$ & -0.137639 & 0.359623 & -1.359986 & $\mathrm{H}$ & 2.434101 & 2.949013 & -1.948289 \\
\hline
\end{tabular}




\begin{tabular}{|c|c|c|c|c|c|c|c|}
\hline $\mathrm{H}$ & 1.026598 & 3.944753 & -1.650170 & $\mathrm{C}$ & 3.098319 & 3.122439 & 0.654381 \\
\hline $\mathrm{H}$ & -3.061121 & 3.746084 & -1.584769 & $\mathrm{C}$ & 3.380722 & 2.231796 & -0.552876 \\
\hline $\mathrm{H}$ & 4.997928 & -2.617953 & -0.457690 & 0 & 2.258241 & 4.186941 & 0.308413 \\
\hline & nergy $=-193$ & 38.5893483 & 7 a.u. & 0 & 4.204014 & 1.232801 & -0.239143 \\
\hline & & & & 0 & 2.924482 & 2.410536 & -1.653048 \\
\hline & $, 10 R, 13 S, 24$ & $R)-\mathbf{1}$, Conf & & $\mathrm{C}$ & 4.568177 & 0.349754 & -1.310343 \\
\hline & & & & $\mathrm{C}$ & 1.298520 & -4.663174 & 0.113282 \\
\hline $\mathrm{C}$ & 1.368057 & -2.268279 & -1.966563 & $\mathrm{C}$ & 2.291600 & -3.037956 & 1.697396 \\
\hline $\mathrm{C}$ & 2.192079 & -2.434878 & -0.702574 & $\mathrm{C}$ & -1.467087 & -3.401304 & -1.191520 \\
\hline $\mathrm{C}$ & 1.440280 & -3.167873 & 0.423481 & 0 & 3.410583 & -3.129923 & -0.969584 \\
\hline $\mathrm{C}$ & 0.089671 & -2.420653 & 0.646534 & $\mathrm{H}$ & 0.400943 & -1.418374 & 0.972417 \\
\hline $\mathrm{C}$ & -0.794926 & -2.149494 & -0.614122 & 0 & -4.471848 & 2.631383 & -0.095716 \\
\hline $\mathrm{C}$ & 0.085281 & -1.494158 & -1.692101 & $\mathrm{H}$ & 1.153557 & -3.249845 & -2.398119 \\
\hline $\mathrm{C}$ & -0.736535 & -2.994233 & 1.801696 & $\mathrm{H}$ & 1.960815 & -1.727564 & -2.712943 \\
\hline $\mathrm{C}$ & -1.888535 & -2.060437 & 2.178514 & $\mathrm{H}$ & 2.431451 & -1.429451 & -0.318528 \\
\hline $\mathrm{C}$ & -2.714423 & -1.715956 & 0.970232 & $\mathrm{H}$ & 0.348001 & -0.478114 & -1.368294 \\
\hline $\mathrm{C}$ & -1.890644 & -1.123101 & -0.152062 & $\mathrm{H}$ & -0.471341 & -1.394951 & -2.627372 \\
\hline $\mathrm{C}$ & -4.016231 & -1.976201 & 0.910482 & $\mathrm{H}$ & -0.105671 & -3.140743 & 2.679579 \\
\hline $\mathrm{C}$ & -2.717748 & -0.544713 & -1.311268 & $\mathrm{H}$ & -1.140813 & -3.976228 & 1.541067 \\
\hline $\mathrm{C}$ & -3.723566 & 0.526599 & -0.960209 & $\mathrm{H}$ & -2.514506 & -2.512533 & 2.950659 \\
\hline $\mathrm{C}$ & -3.301249 & 1.904040 & -0.387671 & $\mathrm{H}$ & -1.466820 & -1.137664 & 2.597980 \\
\hline 0 & -4.903443 & 0.382794 & -1.198572 & $\mathrm{H}$ & -1.320243 & -0.296836 & 0.282606 \\
\hline $\mathrm{C}$ & -2.386429 & 2.665955 & -1.372125 & $\mathrm{H}$ & -4.621296 & -1.763337 & 0.037903 \\
\hline $\mathrm{C}$ & -2.473783 & 1.829622 & 0.877664 & $\mathrm{H}$ & -4.523080 & -2.429304 & 1.756311 \\
\hline $\mathrm{C}$ & -1.294771 & 2.442751 & 0.786615 & $\mathrm{H}$ & -2.035089 & -0.106370 & -2.043559 \\
\hline $\mathrm{C}$ & -1.135432 & 2.983371 & -0.583917 & $\mathrm{H}$ & -3.283661 & -1.322384 & -1.824767 \\
\hline 0 & -0.171035 & 3.584544 & -1.009752 & $\mathrm{H}$ & -2.118511 & 2.117337 & -2.276230 \\
\hline $\mathrm{C}$ & -0.238749 & 2.584739 & 1.826795 & $\mathrm{H}$ & -2.878391 & 3.589319 & -1.677180 \\
\hline $\mathrm{S}$ & 1.086049 & 1.347026 & 1.531501 & $\mathrm{H}$ & -2.876590 & 1.358267 & 1.768115 \\
\hline $\mathrm{C}$ & 2.596354 & 2.307741 & 1.847347 & $\mathrm{H}$ & -0.652961 & 2.434433 & 2.822066 \\
\hline
\end{tabular}




\begin{tabular}{|c|c|c|c|c|c|c|c|}
\hline $\mathrm{H}$ & 0.211517 & 3.575180 & 1.773839 & $\mathrm{C}$ & -1.360304 & 1.955341 & -2.334831 \\
\hline $\mathrm{H}$ & 2.427844 & 2.974482 & 2.692704 & $\mathrm{C}$ & -2.332645 & 1.855092 & -1.192358 \\
\hline $\mathrm{H}$ & 3.353344 & 1.580073 & 2.137998 & $\mathrm{C}$ & -1.704351 & 1.332635 & 0.083219 \\
\hline $\mathrm{H}$ & 4.058013 & 3.559899 & 0.948476 & $\mathrm{C}$ & -3.598997 & 2.238948 & -1.318845 \\
\hline $\mathrm{H}$ & 1.493786 & 3.861946 & -0.200902 & $\mathrm{C}$ & -2.720408 & 1.056827 & 1.199677 \\
\hline $\mathrm{H}$ & 5.115323 & -0.467061 & -0.847609 & $\mathrm{C}$ & -3.784421 & 0.021307 & 0.920238 \\
\hline $\mathrm{H}$ & 3.674494 & -0.019912 & -1.813641 & $\mathrm{C}$ & -3.446701 & -1.447287 & 0.551670 \\
\hline $\mathrm{H}$ & 5.198206 & 0.873515 & -2.028702 & 0 & -4.960114 & 0.285245 & 1.055576 \\
\hline $\mathrm{H}$ & 2.271870 & -5.149476 & 0.192034 & $\mathrm{C}$ & -2.483366 & -2.109525 & 1.555864 \\
\hline $\mathrm{H}$ & 0.917931 & -4.863963 & -0.887126 & $\mathrm{C}$ & -2.727786 & -1.591909 & -0.777224 \\
\hline $\mathrm{H}$ & 0.628834 & -5.143617 & 0.829522 & $\mathrm{C}$ & -1.591978 & -2.283702 & -0.698448 \\
\hline $\mathrm{H}$ & 3.318456 & -3.341802 & 1.488456 & $\mathrm{C}$ & -1.346280 & -2.646664 & 0.717015 \\
\hline $\mathrm{H}$ & 2.306615 & -2.004753 & 2.058909 & 0 & -0.395756 & -3.270005 & 1.142691 \\
\hline $\mathrm{H}$ & 1.918129 & -3.674661 & 2.501016 & $\mathrm{C}$ & -0.645731 & -2.652023 & -1.788334 \\
\hline $\mathrm{H}$ & -0.748040 & -4.070608 & -1.659431 & S & 0.800004 & -1.520276 & -1.749430 \\
\hline $\mathrm{H}$ & -2.007896 & -3.969905 & -0.434792 & $\mathrm{C}$ & 2.205803 & -2.638470 & -2.027510 \\
\hline $\mathrm{H}$ & -2.186045 & -3.124152 & -1.965787 & $\mathrm{C}$ & 2.736693 & -3.321465 & -0.768043 \\
\hline $\mathrm{H}$ & 3.810319 & -2.745915 & -1.754234 & $\mathrm{C}$ & 3.215256 & -2.266960 & 0.230785 \\
\hline $\mathrm{H}$ & -5.206931 & 2.111652 & -0.459644 & 0 & 1.855096 & -4.266557 & -0.227428 \\
\hline \multicolumn{4}{|c|}{$\omega \mathrm{B} 97 \mathrm{X}$ Energy = -1938.58934091 a.u. } & 0 & 2.775385 & -2.481564 & 1.462592 \\
\hline & & & & 0 & 3.930855 & -1.344955 & -0.082188 \\
\hline \multicolumn{4}{|c|}{$(3 S, 5 R, 9 S, 10 R, 13 S, 24 R)-\mathbf{1}$, Conf I } & $\mathrm{C}$ & 3.180444 & -1.535298 & 2.462498 \\
\hline & & & & $\mathrm{C}$ & 1.975057 & 4.370053 & -0.273521 \\
\hline $\mathrm{C}$ & 1.526132 & 2.261361 & 2.064139 & $\mathrm{C}$ & 2.842221 & 2.433313 & -1.553589 \\
\hline $\mathrm{C}$ & 2.471025 & 2.168434 & 0.878675 & $\mathrm{C}$ & -1.054583 & 3.643861 & 0.899800 \\
\hline $\mathrm{C}$ & 1.921387 & 2.841467 & -0.391098 & 0 & 3.740144 & 2.745298 & 1.190184 \\
\hline $\mathrm{C}$ & 0.499198 & 2.257076 & -0.657248 & $\mathrm{H}$ & 0.691476 & 1.191123 & -0.839143 \\
\hline $\mathrm{C}$ & -0.515880 & 2.256736 & 0.531596 & 0 & -4.658987 & -2.161573 & 0.464512 \\
\hline $\mathrm{C}$ & 0.177032 & 1.626654 & 1.752154 & $\mathrm{H}$ & 1.408701 & 3.308496 & 2.356397 \\
\hline $\mathrm{C}$ & -0.140695 & 2.789547 & -1.941842 & $\mathrm{H}$ & 1.977482 & 1.752327 & 2.922805 \\
\hline
\end{tabular}




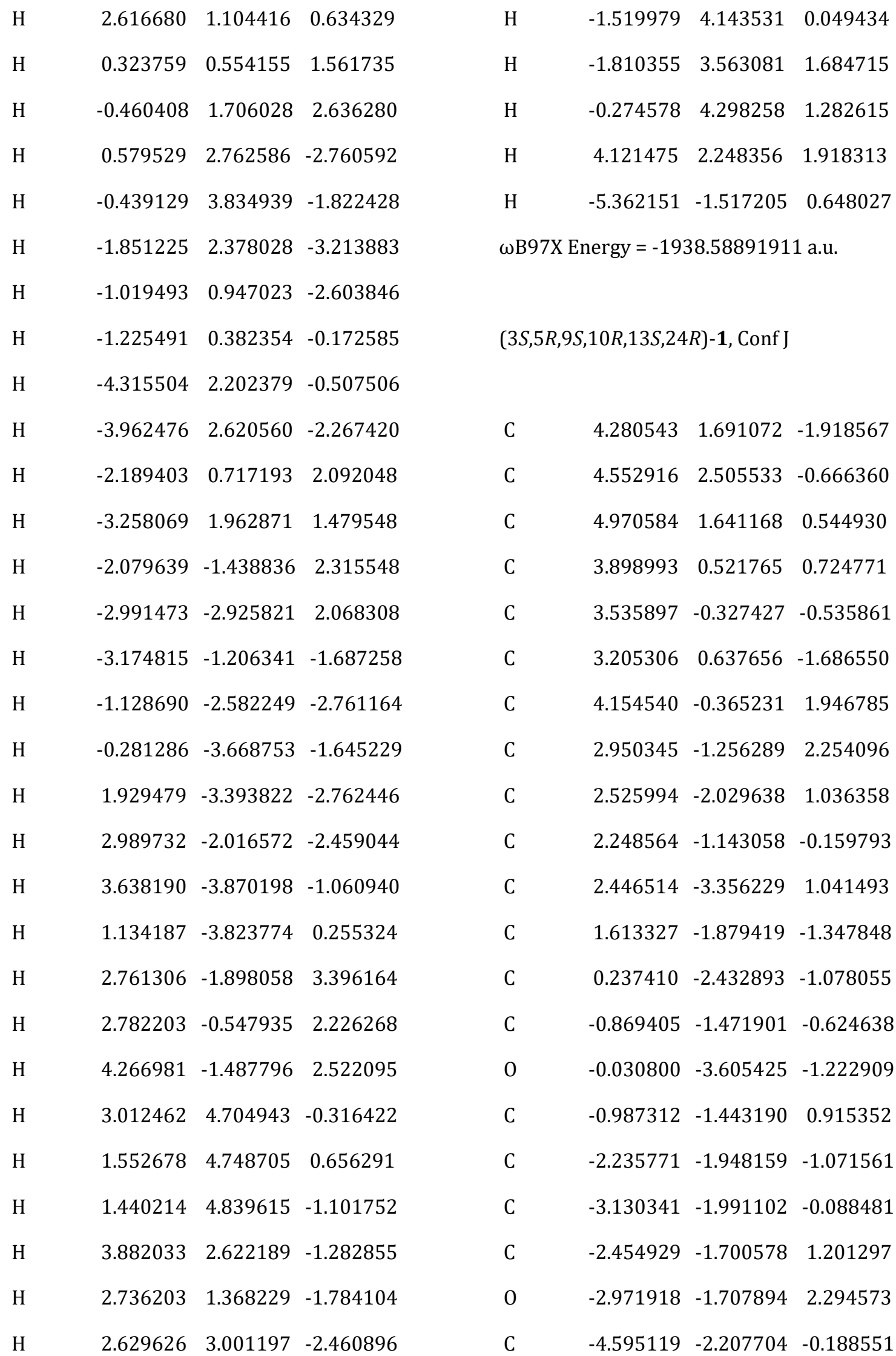




\begin{tabular}{|c|c|c|c|c|c|c|c|}
\hline$S$ & -5.549103 & -0.661297 & 0.037693 & $\mathrm{H}$ & -2.434518 & -2.134421 & -2.121257 \\
\hline $\mathrm{C}$ & -4.896950 & 0.394765 & -1.293404 & $\mathrm{H}$ & -4.948649 & -2.857087 & 0.613539 \\
\hline $\mathrm{C}$ & -4.080371 & 1.588210 & -0.807211 & $\mathrm{H}$ & -4.863530 & -2.657787 & -1.143287 \\
\hline $\mathrm{C}$ & -4.840617 & 2.440173 & 0.200460 & $\mathrm{H}$ & -4.279164 & -0.208735 & -1.958073 \\
\hline 0 & -2.857730 & 1.198311 & -0.218432 & $\mathrm{H}$ & -5.740822 & 0.765559 & -1.873194 \\
\hline 0 & -6.048675 & 2.768159 & -0.214821 & $\mathrm{H}$ & -3.878851 & 2.227289 & -1.675435 \\
\hline 0 & -4.352313 & 2.774865 & 1.254106 & $\mathrm{H}$ & -2.783185 & 1.669457 & 0.624906 \\
\hline $\mathrm{C}$ & -6.831278 & 3.591611 & 0.670683 & $\mathrm{H}$ & -6.327764 & 4.543106 & 0.835031 \\
\hline $\mathrm{C}$ & 6.407560 & 1.127578 & 0.379869 & $\mathrm{H}$ & -7.779734 & 3.742259 & 0.165047 \\
\hline $\mathrm{C}$ & 4.949697 & 2.552008 & 1.782400 & $\mathrm{H}$ & -6.979153 & 3.079646 & 1.620308 \\
\hline $\mathrm{C}$ & 4.639525 & -1.296188 & -0.979562 & $\mathrm{H}$ & 6.599864 & 0.671963 & -0.591296 \\
\hline 0 & 5.483805 & 3.554199 & -0.936388 & $\mathrm{H}$ & 6.647910 & 0.386450 & 1.144084 \\
\hline $\mathrm{H}$ & 2.975516 & 1.079130 & 0.944341 & $\mathrm{H}$ & 7.113986 & 1.952184 & 0.503479 \\
\hline 0 & -0.605401 & -0.197703 & -1.188281 & $\mathrm{H}$ & 5.507284 & 3.466583 & 1.573549 \\
\hline $\mathrm{H}$ & 5.209353 & 1.224260 & -2.264607 & $\mathrm{H}$ & 3.926405 & 2.829440 & 2.052106 \\
\hline $\mathrm{H}$ & 3.964232 & 2.372267 & -2.712453 & $\mathrm{H}$ & 5.412323 & 2.074683 & 2.647963 \\
\hline $\mathrm{H}$ & 3.629283 & 3.026365 & -0.388806 & $\mathrm{H}$ & 4.267601 & -1.956454 & -1.766552 \\
\hline $\mathrm{H}$ & 2.256025 & 1.140819 & -1.464855 & $\mathrm{H}$ & 5.502109 & -0.775584 & -1.390346 \\
\hline $\mathrm{H}$ & 3.055849 & 0.081741 & -2.615456 & $\mathrm{H}$ & 4.989821 & -1.929712 & -0.164054 \\
\hline $\mathrm{H}$ & 4.366851 & 0.251444 & 2.821457 & $\mathrm{H}$ & 6.275505 & 3.163381 & -1.317768 \\
\hline $\mathrm{H}$ & 5.035055 & -0.994006 & 1.788311 & $\mathrm{H}$ & -1.339304 & 0.391019 & -0.937428 \\
\hline $\mathrm{H}$ & 3.176528 & -1.937552 & 3.077177 & \multicolumn{4}{|c|}{$\omega \mathrm{B} 97 \mathrm{X}$ Energy = -1938.58885556 a.u. } \\
\hline $\mathrm{H}$ & 2.116526 & -0.620048 & 2.577206 & & & & \\
\hline $\mathrm{H}$ & 1.523931 & -0.388009 & 0.167631 & \multicolumn{4}{|c|}{$(3 S, 5 R, 9 S, 10 R, 13 S, 24 R)-\mathbf{1}$, Conf $\mathrm{K}$} \\
\hline $\mathrm{H}$ & 2.152183 & -3.932862 & 0.172787 & & & & \\
\hline $\mathrm{H}$ & 2.678091 & -3.915346 & 1.942411 & $\mathrm{C}$ & -1.786875 & 2.260040 & -2.104209 \\
\hline $\mathrm{H}$ & 1.490732 & -1.176349 & -2.174048 & $\mathrm{C}$ & -2.765902 & 1.648464 & -1.118272 \\
\hline $\mathrm{H}$ & 2.228780 & -2.709250 & -1.695783 & $\mathrm{C}$ & -2.537907 & 2.122569 & 0.328287 \\
\hline $\mathrm{H}$ & -0.391576 & -2.218098 & 1.404454 & $\mathrm{C}$ & -1.046471 & 1.825940 & 0.679542 \\
\hline $\mathrm{H}$ & -0.679295 & -0.478285 & 1.320726 & $\mathrm{C}$ & 0.041043 & 2.347263 & -0.314155 \\
\hline
\end{tabular}




\begin{tabular}{|c|c|c|c|c|c|c|c|}
\hline $\mathrm{C}$ & -0.351054 & 1.909324 & -1.736517 & $\mathrm{H}$ & -1.930973 & 3.343069 & -2.145711 \\
\hline $\mathrm{C}$ & -0.683151 & 2.155220 & 2.128747 & $\mathrm{H}$ & -2.003272 & 1.878233 & -3.106861 \\
\hline $\mathrm{C}$ & 0.663176 & 1.538958 & 2.508705 & $\mathrm{H}$ & -2.609353 & 0.556579 & -1.127159 \\
\hline $\mathrm{C}$ & 1.738715 & 1.930487 & 1.533930 & $\mathrm{H}$ & -0.228517 & 0.821458 & -1.822482 \\
\hline $\mathrm{C}$ & 1.384153 & 1.638655 & 0.088557 & $\mathrm{H}$ & 0.317146 & 2.361830 & -2.472880 \\
\hline $\mathrm{C}$ & 2.869978 & 2.499227 & 1.939102 & $\mathrm{H}$ & -1.445637 & 1.771052 & 2.807752 \\
\hline $\mathrm{C}$ & 2.536009 & 1.931564 & -0.881224 & $\mathrm{H}$ & -0.645455 & 3.237282 & 2.281778 \\
\hline C & 3.760309 & 1.058574 & -0.770926 & $\mathrm{H}$ & 0.949304 & 1.831292 & 3.521270 \\
\hline $\mathrm{C}$ & 3.693441 & -0.484780 & -0.885704 & $\mathrm{H}$ & 0.563829 & 0.445745 & 2.502706 \\
\hline 0 & 4.865492 & 1.542850 & -0.653309 & $\mathrm{H}$ & 1.162431 & 0.565387 & 0.039089 \\
\hline $\mathrm{C}$ & 2.713122 & -1.028819 & -1.932168 & $\mathrm{H}$ & 3.659230 & 2.804880 & 1.263522 \\
\hline $\mathrm{C}$ & 3.241656 & -1.155049 & 0.404895 & $\mathrm{H}$ & 3.039815 & 2.683542 & 2.994788 \\
\hline $\mathrm{C}$ & 2.304329 & -2.085836 & 0.222343 & $\mathrm{H}$ & 2.189198 & 1.824533 & -1.911913 \\
\hline $\mathrm{C}$ & 1.894490 & -2.071659 & -1.202946 & $\mathrm{H}$ & 2.887268 & 2.957711 & -0.776689 \\
\hline 0 & 1.031662 & -2.758762 & -1.712620 & $\mathrm{H}$ & 2.036960 & -0.282283 & -2.351197 \\
\hline $\mathrm{C}$ & 1.779454 & -3.082821 & 1.204729 & $\mathrm{H}$ & 3.256467 & -1.484070 & -2.759327 \\
\hline S & 0.391256 & -2.520518 & 2.247754 & $\mathrm{H}$ & 3.751018 & -0.942091 & 1.338884 \\
\hline $\mathrm{C}$ & -0.899265 & -2.105748 & 1.047434 & $\mathrm{H}$ & 2.557915 & -3.333530 & 1.925172 \\
\hline $\mathrm{C}$ & -1.678110 & -3.292613 & 0.472639 & $\mathrm{H}$ & 1.489173 & -3.997210 & 0.690277 \\
\hline $\mathrm{C}$ & -2.766758 & -2.720855 & -0.436882 & $\mathrm{H}$ & -1.588468 & -1.458095 & 1.592439 \\
\hline 0 & -0.880175 & -4.214740 & -0.213896 & $\mathrm{H}$ & -0.469770 & -1.511396 & 0.236733 \\
\hline 0 & -3.806165 & -2.284029 & 0.269885 & $\mathrm{H}$ & -2.163646 & -3.830653 & 1.288509 \\
\hline 0 & -2.676595 & -2.637755 & -1.636176 & $\mathrm{H}$ & -0.310682 & -3.740850 & -0.843979 \\
\hline $\mathrm{C}$ & -4.875575 & -1.666236 & -0.466396 & $\mathrm{H}$ & -5.636263 & -1.426188 & 0.270724 \\
\hline $\mathrm{C}$ & -2.962817 & 3.586107 & 0.505485 & $\mathrm{H}$ & -4.525179 & -0.755576 & -0.954076 \\
\hline C & -3.442329 & 1.275876 & 1.238800 & $\mathrm{H}$ & -5.267537 & -2.359265 & -1.209618 \\
\hline $\mathrm{C}$ & 0.241522 & 3.867362 & -0.281103 & $\mathrm{H}$ & -2.576383 & 4.248668 & -0.267091 \\
\hline 0 & -4.116585 & 1.923208 & -1.487161 & $\mathrm{H}$ & -2.634016 & 3.970644 & 1.473265 \\
\hline $\mathrm{H}$ & -0.977782 & 0.731966 & 0.596966 & $\mathrm{H}$ & -4.051072 & 3.657542 & 0.473206 \\
\hline 0 & 4.998619 & -0.952230 & -1.160746 & $\mathrm{H}$ & -4.466457 & 1.301102 & 0.863455 \\
\hline
\end{tabular}




\begin{tabular}{|c|c|c|c|c|c|c|c|}
\hline $\mathrm{H}$ & -3.119564 & 0.230804 & 1.268253 & 0 & -3.795258 & 1.614723 & 0.522446 \\
\hline $\mathrm{H}$ & -3.457513 & 1.656174 & 2.261172 & $\mathrm{C}$ & -2.617282 & 1.308299 & -2.270691 \\
\hline $\mathrm{H}$ & 1.054727 & 4.156860 & -0.950663 & S & -1.983808 & -0.401454 & -2.400553 \\
\hline $\mathrm{H}$ & -0.643880 & 4.403673 & -0.614757 & $\mathrm{C}$ & -2.598277 & -1.201241 & -0.894210 \\
\hline $\mathrm{H}$ & 0.494507 & 4.229596 & 0.716110 & $\mathrm{C}$ & -4.085717 & -1.566866 & -0.911752 \\
\hline $\mathrm{H}$ & -4.237717 & 1.647061 & -2.399534 & $\mathrm{C}$ & -4.398764 & -2.333188 & 0.373774 \\
\hline $\mathrm{H}$ & 5.598918 & -0.222804 & -0.940189 & 0 & -4.936208 & -0.464033 & -1.053844 \\
\hline & nergy $=-193$ & 38.5888121 & 6 a.u. & 0 & -3.906095 & -3.568638 & 0.331245 \\
\hline & & & & 0 & -4.987555 & -1.869191 & 1.317229 \\
\hline & $, 10 R, 13 S, 24$ & $4 R)-\mathbf{1}$, Conf $\mathrm{L}$ & & $\mathrm{C}$ & -4.094271 & -4.372807 & 1.506780 \\
\hline & & & & $\mathrm{C}$ & 3.767665 & -2.721274 & -1.065713 \\
\hline $\mathrm{C}$ & 2.558023 & 0.011851 & -2.151692 & $\mathrm{C}$ & 5.765874 & -1.372720 & -0.505575 \\
\hline $\mathrm{C}$ & 4.018055 & -0.356715 & -1.946512 & $\mathrm{C}$ & 1.094499 & -1.379393 & 0.257500 \\
\hline $\mathrm{C}$ & 4.249748 & -1.300325 & -0.742823 & 0 & 4.586558 & -0.863503 & -3.153866 \\
\hline $\mathrm{C}$ & 3.573461 & -0.658724 & 0.507149 & $\mathrm{H}$ & 4.123005 & 0.283963 & 0.649414 \\
\hline $\mathrm{C}$ & 2.086616 & -0.210829 & 0.350560 & 0 & -0.480032 & 4.826907 & 0.133377 \\
\hline $\mathrm{C}$ & 1.970350 & 0.663119 & -0.906920 & $\mathrm{H}$ & 1.984918 & -0.878648 & -2.432761 \\
\hline $\mathrm{C}$ & 3.784066 & -1.467922 & 1.791062 & $\mathrm{H}$ & 2.489824 & 0.701886 & -2.996371 \\
\hline $\mathrm{C}$ & 3.308913 & -0.705556 & 3.030567 & $\mathrm{H}$ & 4.577820 & 0.564354 & -1.747853 \\
\hline $\mathrm{C}$ & 1.905581 & -0.190639 & 2.856860 & $\mathrm{H}$ & 2.490837 & 1.611795 & -0.732553 \\
\hline $\mathrm{C}$ & 1.754904 & 0.659161 & 1.612781 & $\mathrm{H}$ & 0.918608 & 0.899816 & -1.096983 \\
\hline $\mathrm{C}$ & 0.929788 & -0.507838 & 3.700178 & $\mathrm{H}$ & 4.842030 & -1.705305 & 1.913573 \\
\hline $\mathrm{C}$ & 0.430247 & 1.424971 & 1.534935 & $\mathrm{H}$ & 3.256807 & -2.423994 & 1.735416 \\
\hline $\mathrm{C}$ & 0.532788 & 2.811371 & 0.954567 & $\mathrm{H}$ & 3.374511 & -1.340459 & 3.916627 \\
\hline $\mathrm{C}$ & -0.711374 & 3.450032 & 0.292548 & $\mathrm{H}$ & 3.976357 & 0.149654 & 3.191924 \\
\hline 0 & 1.535929 & 3.485176 & 1.018793 & $\mathrm{H}$ & 2.540770 & 1.421343 & 1.667960 \\
\hline $\mathrm{C}$ & -2.018814 & 3.178656 & 1.053661 & $\mathrm{H}$ & -0.092292 & -0.167376 & 3.577781 \\
\hline C & -0.954264 & 2.791078 & -1.050704 & $\mathrm{H}$ & 1.127770 & -1.140968 & 4.558772 \\
\hline $\mathrm{C}$ & -2.079844 & 2.079273 & -1.110627 & $\mathrm{H}$ & -0.359004 & 0.863645 & 1.031885 \\
\hline $\mathrm{C}$ & -2.780539 & 2.196489 & 0.192487 & $\mathrm{H}$ & 0.061276 & 1.603412 & 2.550997 \\
\hline
\end{tabular}




$\begin{array}{lllllllll}\mathrm{H} & -1.909116 & 2.806862 & 2.071804 & \mathrm{H} & 2.753716 & -2.758477 & -1.463908 \\ \mathrm{H} & -2.583177 & 4.111830 & 1.096650 & \mathrm{H} & 3.796331 & -3.355017 & -0.177769 \\ \mathrm{H} & -0.249758 & 2.934804 & -1.863185 & \mathrm{H} & 4.431082 & -3.180165 & -1.803004 \\ \mathrm{H} & -2.300334 & 1.775363 & -3.203246 & \mathrm{H} & 6.274581 & -1.592958 & -1.445592 \\ \mathrm{H} & -3.704654 & 1.283878 & -2.252937 & \mathrm{H} & 6.152809 & -0.424121 & -0.121804 \\ \mathrm{H} & -2.006066 & -2.112756 & -0.799732 & \mathrm{H} & 6.028962 & -2.158930 & 0.204189 \\ \mathrm{H} & -2.375073 & -0.580248 & -0.023361 & \mathrm{H} & 1.290546 & -2.156303 & 0.995999 \\ \mathrm{H} & -4.278577 & -2.233167 & -1.754752 & \mathrm{H} & 0.072974 & -1.030988 & 0.426805 \\ \mathrm{H} & -4.679112 & 0.216954 & -0.409278 & \mathrm{H} & 1.101913 & -1.844916 & -0.726535 \\ \mathrm{H} & -5.157421 & -4.515031 & 1.697204 & \mathrm{H} & 4.056588 & -1.610988 & -3.445833 \\ \mathrm{H} & -3.616432 & -5.323890 & 1.292055 & \mathrm{H} & 0.467599 & 4.964090 & 0.286934 \\ \mathrm{H} & -3.627005 & -3.896807 & 2.368154 & \mathrm{HB} 97 \mathrm{X} & & & & \\ \mathrm{H} & & & & & & & & \end{array}$

\section{Supplementary references.}

(1) MacroModel; Schrödinger, L.L.C., 2015, http://www.schrodinger.com/MacroModel.

(2) Frisch, M. J.; Trucks, G. W.; Schlegel, H. B.; Scuseria, G. E.; Robb, M. A.; Cheeseman, J. R.; Scalmani, G.; Barone, V.; Mennucci, B.; Petersson, G. A.; Nakatsuji, H.; Caricato, M.; Li, X.; Hratchian, H. P.; Izmaylov, A. F.; Bloino, J.; Zheng, G.; Sonnenberg, J. L.; Hada, M.; Ehara, M.; Toyota, K.; Fukuda, R.; Hasegawa, J.; Ishida, M.; Nakajima, T.; Honda, Y.; Kitao, O.; Nakai, H.; Vreven, T.; Montgomery, J. A., Jr.; Peralta, J. E.; Ogliaro, F.; Bearpark, M.; Heyd, J. J.; Brothers, E.; Kudin, K. N.; Staroverov, V. N.; Kobayashi, R.; Normand, J.; Raghavachari, K.; Rendell, A.; Burant, J. C.; Iyengar, S. S.; Tomasi, J.; Cossi, M.; Rega, N.; Millam, J. M.; Klene, M.; Knox, J. E.; Cross, J. B.; Bakken, V.; Adamo, C.; Jaramillo, J.; Gomperts, R.; Stratmann, R. E.; Yazyev, O.; Austin, A. J.; Cammi, R.; Pomelli, C.; Ochterski, J. W.; Martin, R. L.; Morokuma, K.; Zakrzewski, V. G.; Voth, G. A.; Salvador, P.; Dannenberg, J. J.; Dapprich, S.; Daniels, A. D.; Farkas, Ö.; Foresman, J. B.; Ortiz, J. V.; Cioslowski, J.; Fox, D. J. Gaussian 09, Revision B. 01; Gaussian: Wallingford, CT, USA, 2010.

(3) Stephens, P. J.; Harada, N. Chirality. 2010, 22, 229-233.

(4) Varetto, U. MOLEKEL 5.4; Swiss National Supercomputing Centre: Manno, Switzerland, 2009.

(5) Zheng, J.; Yu,Y.; Feng, W.; Li, J.; Liu, J.; Zhang, C.; Dong, Y.; Pessah, IN.; Cao, Z. Environ. Health. Perspect. 2019, 127, 67003.

(6) (a) Kicsák, M.; Mándi, A.; Varga, S.; Herczeg, M.; Batta, G.; Bényei, A.; Borbás, A.; Herczegh, P. Org. Biomol. Chem. 2018, 16, 393-401. (b) Csupor, D.; Kurtán, T.; Vollár, M.; Kúsz, N.; Kövér, K. E.; Mándi, A. Szűcs, P.; Marschall, M.; Tahaei, S. A. S.; Zupkó, I.; Hohmann, J. J. Nat. Prod. 2020, 83, 268-276. 